\title{
THEORETICAL AND COMPUTATIONAL MODELING OF PEPTIDE-LIPID BILAYER INTERACTION STUDIED BY DYNAMIC FORCE SPECTROSCOPY
}

\author{
A Thesis presented to \\ the Faculty of the Graduate School \\ at the University of Missouri
}

In Partial Fulfillment

of the Requirements for the Degree

Doctor of Philosophy

by
MILICA UTJESANOVIC

Dr. IOAN KOSZTIN, Thesis Supervisor

JULY 2019 
The undersigned, appointed by the Dean of the Graduate School, have examined the dissertation entitled:

\section{THEORETICAL AND COMPUTATIONAL MODELING OF PEPTIDE-LIPID BILAYER INTERACTION STUDIED BY DYNAMIC FORCE SPECTROSCOPY}

presented by Milica Utjesanovic, a candidate for the degree of Doctor of Philosophy and hereby certify that, in their opinion, it is worthy of acceptance.

Dr. Ioan Kosztin

Dr. Gavin King

Dr. Shi-Jie Chen

Dr. Carsten Ullrich

Dr. Peter Cornish 


\section{ACKNOWLEDGMENTS}

I would first like to express my immense gratitude to my advisor, Dr. Ioan Kosztin, for all of his offered guidance and mentorship throughout my graduate studies. He has taught me everything I know and has always supported me during my ongoing learning process. His expertise and feedback have led me towards the completion of my projects and this thesis. I would also like to thank Dr. Dorina Kosztin who, even though is not a committee member, has helped me to balance my teaching and research responsibilities.

Also, thank you to all of my committee members: Dr. Shi-Jie Chen, Dr. Carsten Ullrich, Dr. Peter Cornish and especially Dr. Gavin King, whose comments and suggestions have been an important piece of the success of my research projects.

Finally, thank you to Ph.D. candidates Ashutosh Dahal, Edward Pluhar and Matthew Anderson, for their, beyond helpful, discussions and encouragement over the years. 


\section{TABLE OF CONTENTS}

ACKNOWLEDGMENTS ...................... ii

LIST OF TABLES .......................... . . vi

LIST OF FIGURES ........................ . vii

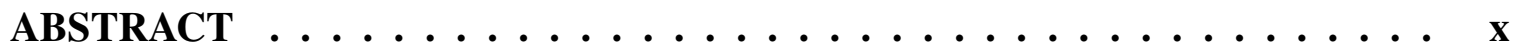

\section{CHAPTER}

1 Introduction .......................... 1

2 Theoretical modeling of the forced detachment of a peptide from a lipid

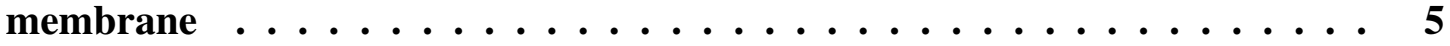

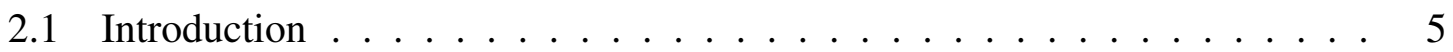

2.2 Theoretical model . . . . . . . . . . . . . . . . . 8

2.2.1 Detachment along a single pathway ............ 9

2.2.2 Detachment along multiple pathways . . . . . . . . 15

2.2.3 Double and multiple detachment events . . . . . . . . . . . . 16

2.3 Conclusions . . . . . . . . . . . . . . . . 17

3 Forced detachment of SecA2-11 from zwitterionic and polar lipid bilayers . . 19

3.1 Detachment of SecA2-11 from zwitterionic POPC bilayer . . . . . . . . . 21

3.1.1 $P(F):$ AFM-tip dependence . . . . . . . . . . . . . 21

3.1.2 $P(F)$ : retraction speed, $v$, dependence . . . . . . . . . 23

3.1.3 $k(F)$ : retraction speed, $v$, dependence . . . . . . . . 25

3.2 Detachment of SecA2-11 from polar E. coli membrane . . . . . . . . . . . 27 
3.2.1 $P(F)$ : dependence on the type of lipid membrane . . . . . . . . 27

3.2.2 $P(F)$ : retraction speed, $v$, dependence . . . . . . . . . . 29

3.2.3 $k(F)$ : retraction speed, $v$, dependence $\ldots \ldots . \ldots . \ldots 31$

3.3 Dissociation rate of SecA2-11 from POPC and E. coli lipid membranes . . . 32

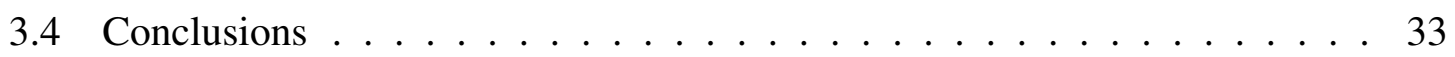

4 MD simulations of SecA2-11 interacting with zwitterionic POPC and charged

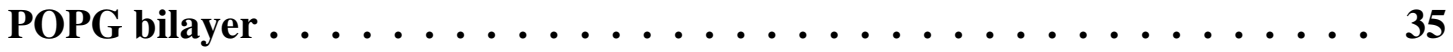

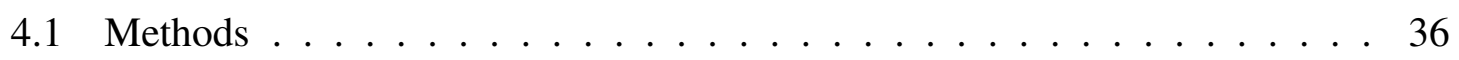

4.1.1 Building the systems .................... 36

4.1.2 MD simulations . . . . . . . . . . . . 37

4.2 Results and Discussion . . . . . . . . . . . . . . . 39

4.2.1 Membrane penetration depth of SecA2-11 . . . . . . . . . . 39

4.2.2 Helical content of $\operatorname{Sec} \mathrm{A} 2-11 \ldots \ldots . \ldots . \ldots 43$

4.3 Conclusions .............................. 45

5 Conformational dynamics and energetics of melittin and its diastereomer interacting with POPC and POPG lipid bilayers: a molecular dynamics study 46

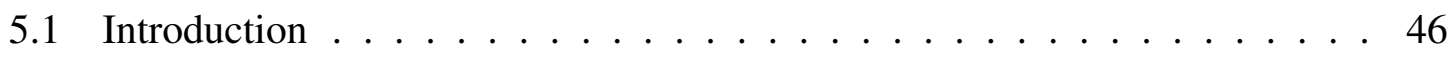

5.2 Methods ............................ 50

5.2.1 Building the systems .................. 50

5.2 .2 MD simulations . . . . . . . . . . . . . . . 52

5.2 .3 Analysis methods .................. 56

5.3 Results and Discussion . . . . . . . . . . . . . 57

5.3 .1 MWT and MD4 in solution . . . . . . . . . . . . . 57 
5.3.2 MD simulations of MWT and MD4 partitioning into POPC lipid

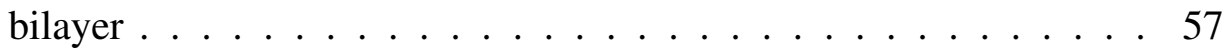

5.3.3 MD simulation of MWT and MD4 partitioning into POPG lipid bilayer . . . . . . . . . . . . . . . . 63

5.3.4 Potential of Mean Force $(\mathrm{PMF}) \ldots \ldots \ldots$

5.4 Conclusions . . . . . . . . . . . . . . . . . 73

6 Summary and concluding remarks $\ldots \ldots \ldots \ldots \ldots \ldots \ldots$ APPENDIX

A Peptide-lipid affinity assay that reveals an interplay between solution structure and partitioning . . . . . . . . . . . . . 79

A.1 Methods . . . . . . . . . . . . . . . . . . . 79

A.2 Results and Discussion $\ldots \ldots \ldots \ldots \ldots \ldots \ldots$

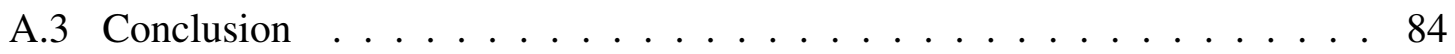

B Isopeptide bond $\ldots \ldots \ldots \ldots \ldots \ldots \ldots \ldots \ldots \ldots$. . . . . . . . . . . .

B.1 Lysine and default peptide bond topology entry $\ldots \ldots \ldots \ldots$. . . 87

B.2 Isopeptide bond patch . . . . . . . . . . . . . . 89

C Umbrella Sampling \& WHAM . . . . . . . . . . . . 91

D MD test runs of MWT and MD4 $\ldots \ldots \ldots \ldots \ldots \ldots$

E Analysis of hydrogen bonds formed between bilayers (POPG/POPC) and peptides (MWT and MD4) $\ldots \ldots \ldots \ldots \ldots \ldots 1$

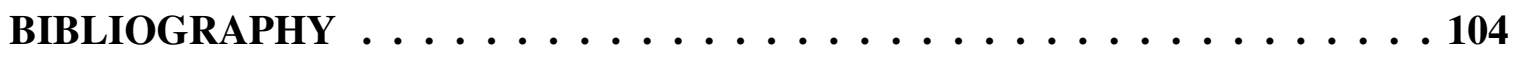

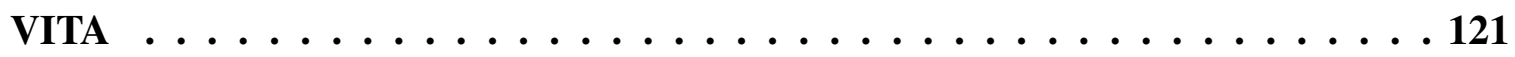




\section{LIST OF TABLES}

Table

Page

3.1 PMF model parameters of selected residues with POPC lipid bilayer . . . 20

4.1 Electrostatic interaction energy $\left(E_{\mathrm{es}}\right)$, solvent accessible surface area (SASA) and radius of gyration $\left(R_{g}\right)$ of SecA2-11 in PC and PG. . . . . . 40

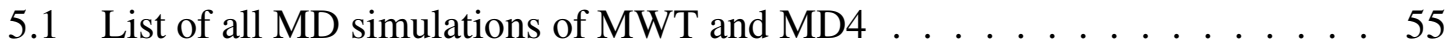




\section{LIST OF FIGURES}

Figure

2.1 Experimental setup and theoretical models . . . . . . . . . . . . . . 10

2.2 Single detachment pathway model fails to describe the experimental $P(F)$ and $k(F)$ for peptide-lipid membrane detachment. . . . . . . . . . 14

3.1 Detachment force histogram of SecA2-11 interacting with POPC bilayer . . 22

3.2 $P(F)$ of SecA2-11 interacting with POPC bilayer for different retraction speeds of the AFM tip . . . . . . . . . . . . . . . . . 24

3.3 Force dependent detachment rate for SecA2-11 interacting with POPC bilayer 25

3.4 Force dependent detachment rate of SecA2-11 from POPC lipid bilayer at $v=100 \mathrm{~nm} / \mathrm{s}$ retraction speed $\ldots \ldots \ldots \ldots \ldots \ldots$

$3.5 P(F)$ for SecA2-11 in POPC bilayer and E. coli membrane . . . . . . . 28

3.6 Retraction speed dependence of $P(F)$ for SecA2-11 in E. coli _ . . . . . 30

$3.7 k(F)$ of SecA2-11 from E. coli membrane . . . . . . . . . . . . . . 31

3.8 The intrinsic detachment rate dependence on AFM retraction speed . . . . . 32

4.1 Selected dynamics parameters of POPC/POPG bilayers after equilibration . 38

$4.2 z(t)$ of the COM of SecA2-11 in POPC and POPG bilayers . . . . . . . 41 
4.3 Penetration depth of SecA2-11, per residue, in PC and PG averaged over total simulation time . . . . . . . . . . . . . . 42

$4.43_{10}$ helical content of SecA2-11 in PG system . . . . . . . . . . . . . 44

5.1 Snapshot showing MWT in POPC bilayer . . . . . . . . . . . . . . . 49

5.2 Snapshot showing the difference between the initial structures of MWT and

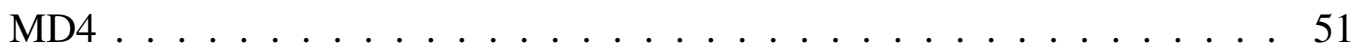

5.3 Initial configuration of the peptide-lipid bilayer system $\ldots \ldots$. . . . . 53

5.4 Helical content of MWT and MD4 in solution . . . . . . . . . . . 58

5.5 Average helical content per residue of MWT and MD4 in solution . . . . . 59

5.6 Average helical content per residue compared between DSSP and "custom" $\operatorname{method} \ldots \ldots \ldots \ldots \ldots$

5.7 The RMSD of MWT and MD4 in aqueous solution . . . . . . . . . 61

5.8 Position of MWT and MD4 relative to the COM POPC bilayer . . . . . . 63

5.9 Helical content of MWT and MD4 in POPC . . . . . . . . . . . . . . . 64

5.10 Position of MWT and MD4 relative to the COM POPG bilayer . . . . . . 66

5.11 Helical content of MWT and MD4 in POPG . . . . . . . . . . . . 67

5.12 PMF for MWT and MD4 in POPC and POPG lipid bilayers . . . . . . . . 69

5.13 Number of hydrogen bonds and helical content of MWT and MD4 in POPC and POPG during US simulations . . . . . . . . . . . . . . . 70

5.14 Number of hydrogen bonds and helical content of MWT and MD4 in POPC, compared between free and US simulations . . . . . . . . . . . 71

5.15 Number of hydrogen bonds and helical content of MWT and MD4 in POPG, compared between free and umbrella sampling (US) simulations . . . . . 72 
A.1 Mechanical protein-lipid interaction assay . . . . . . . . . . . 80

A.2 Peptides solution structures and main results obtained from MD simulations 84

A.3 Radius of gyration for peptide solution structures . . . . . . . . 85

A.4 Solvent accessible surface area (SASA) of SecA2-11 constructs . . . . . . . 85

B.1 Topology entry for Lysine . . . . . . . . . . . . . . . . . . 88

B.2 Topology entry for peptide bond . . . . . . . . . . . . . . 89

B.3 Script for isopepeptide bond (IPB) . . . . . . . . . . . . . . . 90

B.4 Snapshot showing ispopeptide bond between G12' and K13 . . . . . . . 90

D.1 Time evolution of $z$-coordinate for MWT' in POPC . . . . . . . . . . . 98

D.2 Time evolution of helical content for MWT' in POPC . . . . . . . . . . . 98

D.3 Time evolution of $z$-coordinate of MD4' in POPC . . . . . . . . . . 99

D.4 Time evolution of helical content for MD4' in POPC . . . . . . . . . . . . 99

D.5 Time evolution of $z$-coordinate of a single copy of MWT in POPC . . . . 100

E.1 Hydrogen bonds formed between POPC bilayer and peptides (MD4 and

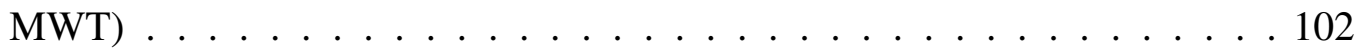

E.2 Hydrogen bonds formed between POPG bilayer and peptides (MD4 and

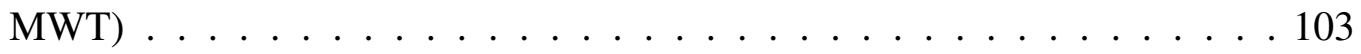




\begin{abstract}
This thesis consists of three interrelated theoretical and computational modeling projects that investigate different aspects of peptide-lipid membrane interactions. (1) A general theoretical approach is formulated for the quantitative description of the detachment force distribution, $P(F)$, and the corresponding force dependent detachment rate, $k(F)$, of a peptide from a lipid bilayer, by assuming that peptide detachment from lipid membranes occurs stochastically along a few dominant diffusive pathways. Besides providing a consistent interpretation of the experimental data, the new method also predicts that $k(F)$ exhibits catch-bond behavior (when, counter intuitively, the detachment rate decreases with increasing force). (2) The proposed multiple detachment pathways method is tested and validated for a particular peptide (SecA2-11) interacting with both zwitterionic POPC lipid and polar E. Coli membranes. Furthermore, molecular dynamics (MD) simulations are used to explored the conformational dynamics of SecA2-11 during its interaction with both POPC and anionic POPG lipid bilayers. (3) Finally, MD simulations are used to explore the conformational dynamics and energetics of the peptide melittin (MWT) and its diastereomer (MD4) interacting with POPC and POPG lipid bilayers. The obtained results provide further insight into the role of secondary structure in peptide-lipid bilayer interactions.
\end{abstract}




\section{Chapter 1}

\section{Introduction}

Peptide-lipid interactions are essential for understanding numerous cellular processes and their mechanisms. Thus, their study is of great conceptual and practical importance in molecular biology and biophysics. In particular, the quantitative description and characterization, at single amino acid level, of the interaction between proteins and lipid membranes is crucial in understanding the partitioning and folding of membrane proteins. Because of the complexity of the system and the wide range of time and length scales involved, both experimental and theoretical study of these interactions is extremely difficult. The nature of peptide-lipid membrane interaction depends significantly on the type and size of the peptide, the species of the lipid, and the properties of the surrounding solution.

Currently, there exist several single-molecule experimental techniques that can be used to measure and characterize (both qualitatively and quantitatively) the interaction of biomolecules with lipid membranes. One such powerful technique is Atomic Force Microscopy (AFM), which can be used to measure, under native conditions, with high precision the force ex-

erted between a biomolecule (e.g., peptide) and a substrate (e.g., lipid membrane) as a 
function of their separation. In general, however, the results obtained in AFM measurements are convoluted and their correct interpretation may require substantial theoretical and/or computational modeling.

This thesis consists of three interrelated theoretical and computational modeling projects, involving peptide-lipid membrane interactions. These projects are part of a joint collaborative effort with Dr. Gavin King and his research group. All AFM data mentioned and used in this thesis are from Dr. King's laboratory. A brief description of the scope and results of these projects follows.

In the first project, described in Ch. 2, we have formulated a general theoretical approach for the quantitative description of the detachment (last-rupture) force distribution, $P(F)$, and the corresponding force dependent detachment rate, $k(F)$, of a peptide from a lipid bilayer [1]. Both $P(F)$ and $k(F)$ can be determined, in a model independent manner, through high-precision, AFM dynamic force spectroscopy measurements. In general, the measured $P(F)$ and $k(F)$, which differ considerably for different peptides, lipid-membranes, AFM tips (prepared under identical conditions), and retraction speeds of the AFM cantilever, cannot be described in terms of the standard theory, according to which detachment occurs along a single pathway, corresponding to a diffusive escape process across a free energy barrier. In particular, the prominent retraction speed dependence of $k(F)$ is a clear indication that peptide-lipid membrane dissociation occurs stochastically along several detachment pathways. The proposed new theoretical approach for $P(F)$ and $k(F)$ assumes that peptide detachment from lipid membranes occurs, with certain probability, along a few dominant diffusive pathways. Besides providing a consistent interpretation of the experimental data, the new method also predicts that for moderate retraction speeds at intermediate force values, $k(F)$ exhibits catch-bond behavior, i.e., when counter intuitively 
the detachment rate decreases with increasing force. According to the proposed model this behavior is due to the stochastic mixing of individual detachment pathways which do not convert or cross during rupture. To our knowledge, such catch-bond mechanism has not been proposed and demonstrated before for a peptide-lipid interaction.

Next, in the second project, described in Chs. 3-4, the proposed multiple detachment pathways method is tested and validated for a particular peptide, i.e., SecA2-11, interacting with two types of lipid bilayers, namely, zwitterionic POPC and polar E. Coli membrane $[1,2]$. In all cases, under various experimental conditions, the agreement between the experimental and theoretical results is surprisingly good. Furthermore, to gain additional insight into the conformational dynamics of SecA2-11 during its interaction with zwitterionic POPC and anionic POPG lipid bilayers, we have performed unbiased, long time scale, all-atom MD simulations. The obtained results are consistent with the mentioned AFM measurements and with other recent experimental results.

Finally, in the third project, described in Ch. 5, in order to directly assess the role of helical secondary structure in peptide-lipid bilayer interactions, all-atom MD simulations are used to explore the conformational dynamics and energetics of the peptide melittin (MWT) and its diastereomer (MD4) interacting respectively with a POPC and a POPG lipid bilayer. In agreement with previous experimental studies [3], MWT appears to be folded and bounded to both membranes, while MD4 is unfolded (random coil) with clear binding affinity only towards the charged POPG membrane. These results are also consistent with the dissociation energies of MWT and MD4 (oriented parallel to the surface of the membrane) from POPC and POPG, extracted from the potentials of mean force (PMFs) determined from umbrella sampling simulations. It should be noted that although there exist many previous MD studies of MWT and several experimental studies of MD4, to the 
best of our knowledge, this is the first long time scale, all-atom MD simulations study of the MD4 peptide. 


\section{Chapter 2}

\section{Theoretical modeling of the forced detachment of a peptide from a lipid membrane $^{1}$}

\subsection{Introduction}

Peptide-lipid membrane interactions are fundamental in molecular biology, by directly affecting the dynamics and functions of membrane proteins [4-6]. Currently, there exist several single-molecule experimental techniques that can be used to measure and characterize (both qualitatively and quantitatively) the interaction of biomolecules with lipid membranes [7]. Atomic force microscopy (AFM) based dynamic force spectroscopy [2, 8,9] is a powerful experimental technique for measuring peptide lipid interaction with sub $\mathrm{pN}$ precision [10]. In a typical, AFM force spectroscopy experiment (see Fig. 2.1a), the peptide, which is attached through a linker to the functionalized tip of the AFM cantilever, is

\footnotetext{
${ }^{1}$ The content of this chapter is based on M. Utjesanovic et al, Scientific Reports 9, 451 (2019) [1].
} 
repeatedly approached to and then retracted (until total detachment) from the surface of the lipid membrane. The cantilever is moved with an adjustable constant speed, $v$. The measured detachment force $F$ (i.e., last rupture force) at the end of each retraction is a stochastic quantity characterized by a distribution function $P(F)$.

The standard approach to interpret the measured histogram, $P(F)$, is to model the peptide-lipid membrane detachment as a diffusive escape process across a free energy barrier [11-14]. Such single detachment pathway model is characterized by (i) an energy parameter (activation energy or barrier height $\Delta U_{0}$ ), (ii) a geometric parameter (activation length $\Delta x_{0}$ ), and (iii) a kinetic parameter (intrinsic escape rate $k_{0}$, or equivalently, escape time $\tau_{0}=1 / k_{0}$ ), as illustrated schematically in Fig. 2.1b. In general, an externally applied detachment force, $F$, oriented normal to the surface of the membrane (along the $x$-axis in this case), will alter all three model parameters such that $\Delta U(F)<\Delta U_{0}, \Delta x(F)<\Delta x_{0}$, and $k(F)>k_{0}$.

Furthermore, $P(F)$ can be determined, in a model independent manner, from the force dependent detachment rate, $k(F)$. The converse is also true (see Eqs. 2.8-2.9). Thus, the values of the model parameters $\left(\Delta U_{0}, \Delta x_{0}, k_{0}\right)$ can be determined by fitting the theoretical rupture force distribution, $P(F)$, to the corresponding experimental histogram.

For the single detachment pathway model, $k(F)$ is independent of $v$ and increases monotonically with $F[14,15]$. Thus, one expects that when this model is applicable then the $k(F)$ s obtained from the experimental $P(F)$ histograms corresponding to different $v$ values should collapse to a single curve that does not depend on $v$. Indeed, this is the case for systems involving force-induced molecular transitions (e.g., unfolding of proteins [16-21], unzipping of nucleic acid hairpins [22,23], ligand-receptor dissociation [24, 25], etc.).

By contrast, for peptide-lipid membrane systems the experimental $P(F)$ histograms 
show noticeable differences even for otherwise identically prepared samples, and depend strongly on other experimental factors, such as the retraction speed, $v$. Likewise, the corresponding rupture rates, $k(F)$, are strongly $v$-dependent and can be non-monotonic functions of $F$. Thus, it is clear that the single detachment pathway model fails for peptide-lipid membrane systems, most likely due to their complexity. Thus, it is logical to assume that for such systems multiple detachment pathways are in play.

Our goal is to propose a general, multi-pathway model for describing the detachment force distribution, $P(F)$, and the corresponding $k(F)$, of forced detachment of peptides from lipid membranes. The proposed model assumes that the detachment process can proceed stochastically along a few, $N>1$, dominant pathways, with probabilities $w_{n}, n=1, \ldots, N$, such that $\sum_{n=1}^{N} w_{n}=1$. Similarly to the standard theory $[14,15]$, each pathway is modeled as a diffusive escape process across a free energy barrier, characterized by the parameters $\Delta U_{0 n}, \Delta x_{0 n}$ and $k_{0 n}$. We identify these pathways as final (last) rupture events involving either one or two residues of the peptide. Indeed, due to its polymeric nature, the separation of the peptide from the membrane during retraction proceeds residue-by-residue. Right before detachment, the peptide-lipid membrane contact is restricted to a single residue, in general, located at the end of the peptide away from the AFM tip. Thus, depending on the time resolution of the AFM instrument, $F$ extracted from the retraction force time series, corresponds to the dissociation of the last residue, or the dissociation in rapid succession of the last two (or more) residues still in contact with the membrane.

Approximate values of energetic $\left(\Delta U_{0 n}\right)$ and geometric $\left(\Delta x_{0 n}\right)$ parameters, peptide residue and lipid species specific, can be obtained from previous free energy profile studies of individual residues interacting with model lipid membranes [26,27]. Thus, only the intrinsic detachment rates $k_{0 n}$ and the pathway weight factors $w_{n}$ are left to be determined as effec- 
tive fitting parameters. Once all the parameters have been determined for a specific peptide and lipid-membrane system, by changing experimental conditions (e.g., modifying the retraction speed $v$, or exchanging - otherwise identically prepared - AFM tips) one only needs to adjust the weight coefficients $w_{n}$ in order to match the experimental $P(F)$ and $k(F)$ with the theoretical model. In general, the probability that detachment follows a certain pathway should change by modifying the experimental conditions.

The viability of the proposed model is demonstrated by applying it to AFM retraction experiments of peptide rupturing from two species of lipid bilayers and performed under different experimental conditions (see Ch. 3). In spite of its formal simplicity, our theoretical model is capable of describing, and interpreting in a consistent manner both $P(F)$ and $k(F)$ for the considered AFM retraction experiments [1]. In addition, we find that for intermediate retractions speeds and rupture forces, $k(F)$ exhibits "catch-bond" behavior [28-30] when, counter intuitively, the detachment rate decreases with increasing $F$. We show that this behavior is due to properly weighted, force dependent contributions by different detachment pathways to $k(F)$. We also find that the intrinsic (detachment-) off-rate, $k_{0}=k(0)=\sum_{n=1}^{N} w_{n} k_{0 n}$, of the peptide from the membrane (within reasonable numerical errors) is independent of $v$, in spite of the fact that the weights $w_{n}$ are $v$-dependent while $k_{n 0}$ are not.

\subsection{Theoretical model}

The forced detachment of a short peptide (biomolecule) from a lipid membrane (substrate), observed in AFM single-molecule dynamic force spectroscopy experiments (Fig. 2.1a), is a stochastic process that customarily is modeled as a diffusive escape event across a free 
energy barrier [11-14]. The reaction coordinate, $x$, is defined as the separation between peptide and membrane, along the normal direction to the latter. In the absence of a pulling force, $F$, the intrinsic free energy profile (potential of mean force or PMF), $U_{0}(x)$, is characterized by the separation $\Delta x_{0}=x_{\cap}-x_{\cup}$ between the positions of the equilibrium bound state $\left(x_{\cup}\right)$ and transition state $\left(x_{\cap}\right)$, and the barrier height (activation energy), $\Delta U_{0}$, separating these two states (see Fig 2.1b). Here we model the PMF by a widely used linear-cubic potential $U_{0}(x)=\Delta U_{0}\left[(3 / 2)\left(x / \Delta x_{0}\right)-2\left(x / \Delta x_{0}\right)^{3}\right]$, which was shown to be suitable for studying forced detachment processes [14]. In addition, the dynamics of the detachment process also depends on an effective diffusion coefficient, $D$, or, equivalently, an intrinsic escape rate $k_{0}$, or escape time $\tau_{0}=1 / k_{0}$.

In a constant pulling speed (force-ramp) AFM experiment, the applied force, $F(t)$, modifies the PMF, i.e., $U(x \mid F)=U_{0}(x)-F x$, thus facilitating the detachment (last rupture) process by reducing both the barrier height, $\Delta U(F)<\Delta U_{0}$, and separation, $\Delta x(F)<\Delta x_{0}$ (Fig. 2.1b). The stochastic process $x(t)$ starts at (or about) $x_{\cup}$ and terminates with detachment, when it reaches the absorbing boundary at $x_{\cap}$. The corresponding detachment force is also a stochastic quantity whose distribution function, $P(F)$, can be constructed from repeated AFM retraction experiments. Our goal is to determine the model parameters $\Delta U_{0}$, $\Delta x_{0}$ and $k_{0}$ that reproduce $P(F)$ obtained from single-molecule AFM force spectroscopy experiments. The theoretical approach for determining $P(F)$ requires several approximations that are discussed below.

\subsubsection{Detachment along a single pathway}

The key quantity for modeling molecular detachment processes along a given pathway is the survival probability (i.e., the probability that rupture still has not yet occurred at time $t$ ) 

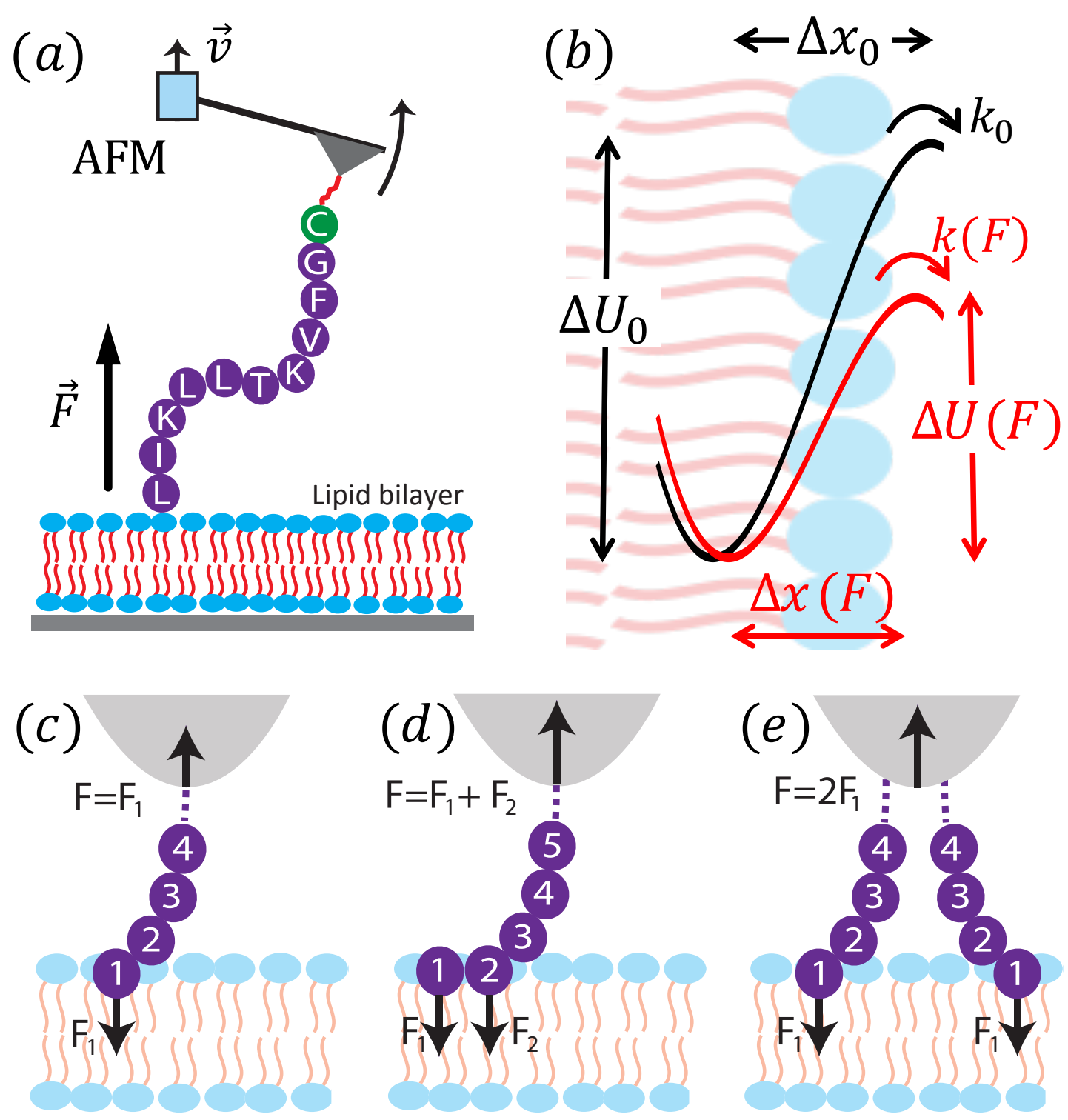

Figure 2.1: (a) Experimental setup. (b) Physical model. Peptide detachment involves the (c) last or next-to-last AA, (d) last two AA or occasionally (e) last two AA on a same AFM tip. 
defined as

$$
S\left(t \mid x_{0}\right)=\int_{a}^{b} p\left(x, t \mid x_{0}\right) d x
$$

where $p\left(x, t \mid x_{0}\right)$ is the transition probability that the stochastic process $x(t)$ has the value $x$ (with $a \leq x \leq b$ ) at time $t$, assuming that at $t=0$ it had the sharp value $x_{0}$. In our case $x_{0}=x_{\cup}$, with an absorbing and reflective boundary at $b=x_{\cap}$ and $a<x_{\cup}$, respectively. When $U(x)$ diverges rapidly for $x<x_{\cup}$, one can set $a=-\infty$.

The transition probability obeys the adjoint Smoluchowski equation [31],

$$
\partial_{t} p\left(x, t \mid x_{0}\right)=\mathcal{L}_{S}^{\dagger}\left(x_{0}\right) p\left(x, t \mid x_{0}\right)
$$

with the adjoint Smoluchowski operator

$$
\mathcal{L}_{S}^{\dagger}\left(x_{0}\right)=D \exp \left[\beta U\left(x_{0}\right)\right] \partial_{x_{0}} \exp \left[-\beta U\left(x_{0}\right)\right] \partial_{x_{0}}=D \partial_{x_{0}}^{2}-\beta D U^{\prime}\left(x_{0}\right) \partial_{x_{0}}
$$

where $\beta=1 / k_{B} T, k_{B}$ is the Boltzmann constant and $T$ is the absolute temperature. Thus, by combining Eqs. 2.1 and 2.2, the equation of motion for the survival probability, subject to the initial condition $S\left(0 \mid x_{0}\right)=1$ and boundary condition $S\left(t \mid x_{\cap}\right)=0$, becomes

$$
\dot{S}\left(t \mid x_{0}\right) \equiv \partial_{t} S\left(t \mid x_{0}\right)=\mathcal{L}_{S}^{\dagger}\left(x_{0}\right) S\left(t \mid x_{0}\right)
$$

In general, $S\left(t \mid x_{0}\right)$ can be calculated only numerically. However, a practical analytical approximation can be obtained by employing the mean-first-passage-time (MFPT) or meandetachment time (MDT) [31] approximation. To this end, note that the distribution function 
of the detachment time is given by $\mathcal{P}(t)=-\dot{S}\left(t \mid x_{0}\right)$. Thus, the MFPT is

$$
\tau\left(x_{0}\right)=\int_{0}^{\infty} t \mathcal{P}(t) d t=\int_{0}^{\infty} S\left(t \mid x_{0}\right) d t
$$

Consequently,

$$
\mathcal{L}_{S}^{\dagger}\left(x_{0}\right) \tau\left(x_{0}\right)=\int_{0}^{\infty} \mathcal{L}_{S}^{\dagger}\left(x_{0}\right) S\left(t \mid x_{0}\right) d t=\int_{0}^{\infty} \dot{S}\left(t \mid x_{0}\right) d t=-1
$$

This is the well-known MFPT equation, which can be integrated exactly with the result [31]

$$
\tau\left(x_{0}\right)=D^{-1} \int_{z}^{x_{n}} d x \exp [\beta U(x)] \int_{a}^{x} d y \exp [-\beta U(y)] .
$$

Clearly, the (mean) detachment time $\tau=\tau\left(x_{\cup}\right)$. Note that in the large barrier limit, Eq. (2.6) turns into the widely used Kramers' formula for the escape time [12, 14, 32,33].

In general, quasi-adiabatic MDT approximation is only applicable for $\tau$ much larger than the characteristic relaxation time of the unruptured system. In single-molecule dynamic force AFM experiments, this approximation usually holds for sufficiently small force loading rates, $\dot{F}=d F / d t$. For simplicity, here only constant loading rates are considered, i.e. $\dot{F}=k_{s} v$, where $k_{s}$ is the stiffness of the AFM cantilever and $v$ is the constant retraction speed. The presence of the linker between the tip of the cantilever and peptide can lead to a weak force dependence of $\dot{F}$. In many cases this dependence can be either neglected or accounted for through a mean spring constant $k_{s}^{*} \sim k_{s}$.

In the MDT approximation, the equation of motion for the survival probability is simplified

$$
\dot{S}(t)=\mathcal{L}_{S}^{\dagger} S(t) \approx-\tau^{-1} S(t)=-k S(t),
$$


and it can be integrated with the result

$$
S(t)=\exp \left(-\int_{0}^{t} k d t^{\prime}\right)
$$

Finally, in terms of the survival probability, the rupture force distribution is $P(F)=$ $-d S / d F=-\dot{S} / \dot{F}$, which in the MDT approximation becomes

$$
P(F)=\frac{k(F)}{\dot{F}} S(F)=\frac{k(F)}{\dot{F}} \exp \left[-\int_{0}^{F} \frac{k(f)}{\dot{F}} d f\right],
$$

where the force dependent detachment rate $k(F)=1 / \tau\left(x_{\cup}\right)$, with the MDT given by Eq. (2.6). Finally, $k(F)$ can be expressed from Eq. (2.8) only in terms of the experimental histogram $P(F)$ and force loading rate in a model independent manner, i.e.,

$$
k(F)=\frac{\dot{F} P(F)}{1-\int_{0}^{F} P(f) d f} .
$$

Either of the last two equations can be used to determine the sought model parameters $\Delta U_{0}$, $\Delta x_{0}$ and $k_{0}$, by fitting the theoretical prediction to the experimental data. However, Eq. (2.9) is crucial in determining whether the detachment process follows a single pathway or multiple pathways. Indeed, in the case of a single detachment pathway, $k(F)$ is independent of the retraction speed $v$. Thus, $k(F \mid v)$ obtained from AFM experiments using different retraction speeds must collapse to the same, monotonically increasing curve. [14] On the other hand, as shown in Fig. 2.2, whenever $k(F \mid v)$ shows significant $v$ dependence (and possibly non-monotonic behavior in $F$ ) it is a clear indication that the detachment process occurs stochastically along several different pathways. 

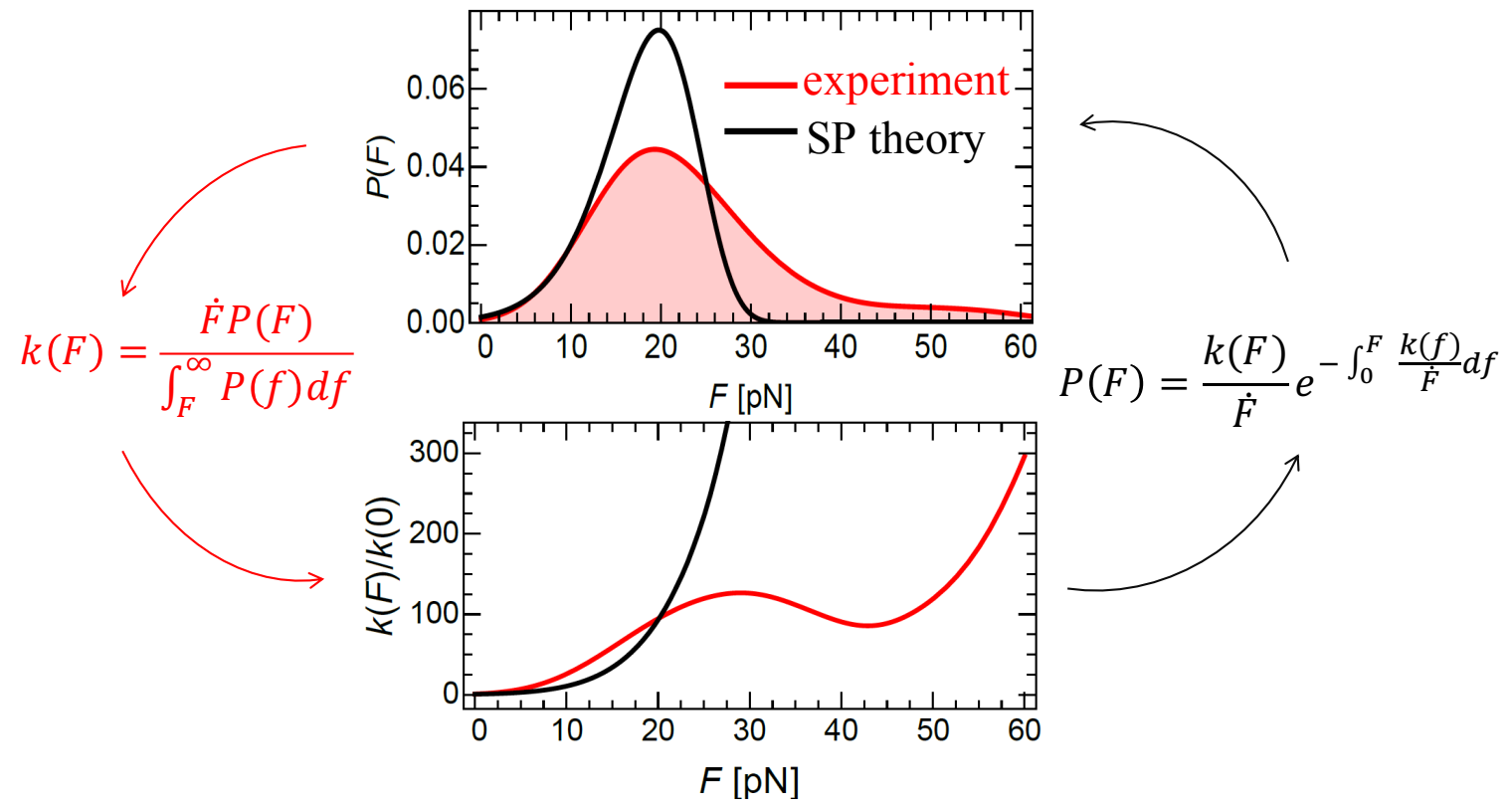

Figure 2.2: In a typical case of peptide-lipid bilayer detachment, the experimentally determined $P(F)$ and $k(F)$ (red curves) cannot be described by a theoretical model based on a single detachment pathway (black curves). The experimental data correspond to the peptide SecA2-11 interacting with a POPC bilayer, for $v=100 \mathrm{~nm} / \mathrm{s}$. 


\subsubsection{Detachment along multiple pathways}

It is reasonable to assume that the detachment of a peptide from a lipid membrane, due to the complex, stochastic nature of their interaction, may follow a number of different, independent pathways. In such case, the probability that detachment has not occurred yet at time $t$ (survival probability) can be written as a weighted $\operatorname{sum} S(t)=\sum_{n=1}^{N} w_{n} S_{n}(t)$, where $w_{n}$ is the probability of detachment along the $n$-th pathway. Clearly, $\sum_{n=1}^{N} w_{n}=1$. Each detachment pathway is characterized by the parameters $\Delta U_{0 n}, \Delta x_{0 n}$ and $k_{0 n}, n=1, \ldots, N$. Using the MDT approximation described in the previous section, it is straightforward to show that, in the case of multiple pathways, the detachment force distribution can be written

$$
P(F)=-\frac{d S}{d F}=\sum_{n=1}^{N} w_{n} P_{n}(F),
$$

where for the $n$-th pathway

$$
P_{n}(F)=\frac{k_{n}(F)}{\dot{F}} S_{n}(F),
$$

with

$$
S_{n}(F)=\exp \left(-\int_{0}^{F} \frac{k_{n}(f)}{\dot{F}} d f\right) .
$$

Similarly to Eq. (2.6), the force dependent detachment rate for the $n$-th pathway

$$
k_{n}(F)=D_{n}\left(\int_{x_{n} \cup}^{x_{n \cap}} d x \exp \left[\beta U_{n}(x)\right] \int_{-\infty}^{x} d y \exp \left[-\beta U_{n}(y)\right]\right)^{-1},
$$

and the corresponding intrinsic detachment rate $k_{0 n}=k_{n}(0)$. Equations (2.10), together with Eq. (2.11), are the multiple pathways equivalent of Eq. (2.8), and can be used to determine the model parameters $\Delta U_{0 n}, \Delta x_{0 n}$ and $k_{0 n}$, along with the weight factors $w_{n}$, by fitting the experimental data. 
Finally, the mean, force dependent, detachment rate for multiple pathways is given formally by the same Eq. (2.9) as for a single detachment pathway, except that for $P(F)$ one needs to use Eqs. (2.10). Thus

$$
k(F)=\sum_{n=1}^{N} \alpha_{n}(F) k_{n}(F),
$$

where

$$
\alpha_{n}(F)=\frac{w_{n} S_{n}(F)}{\sum_{n=1}^{N} w_{n} S_{n}(F)}
$$

Depending on the pathways involved, due to the force dependence of the $\alpha_{n}(F)$ coefficients, $k(F)$ may depend strongly on the retraction speed, $v$, and may also exhibit non-monotonic dependence (i.e., catch-bond behavior [28]) on the detachment force, $F$.

\subsubsection{Double and multiple detachment events}

It is reasonable to assume that right before detachment, the contact between a peptide and lipid membrane is confined to a single amino acid (Fig. 2.1c). The experimentally recorded detachment (last rupture) force thus corresponds to such situation. However, due to the limited time resolution of the AFM or other experimental complications, occasionally the recorded detachment force corresponds to a double rupture event, e.g., when either the last two amino acids of a single peptide (Fig. 2.1d), or the last amino acids of two copies of the peptide attached to the same tip of the AFM cantilever (Fig. 2.1e), in contact with the membrane, rupture in rapid succession. In this double-residue rupture case, the measured detachment force is roughly twice as big as for the corresponding single-residue detachment event. Thus, double rupture events can be regarded as independent detachment pathways with a weight factor $w_{m}^{(2)}$ and a rupture force distribution $P_{m}^{(2)}(F)=(1 / 2) P_{m}(F / 2)$, where 
$P_{m}(F) \equiv P_{m}^{(1)}(F)$ corresponds to single residue detachment. In principle, one may consider multiple detachment events that involve the rupture in rapid succession (that cannot be time-resolved experimentally) of $p>2$ amino acids, which can be modeled as pathways with $w_{n}^{(p)}$ and $P_{m}^{(p)}(F)=(1 / p) P_{m}(F / p)$. However, the occurrence of multiple detachment events with $p>2$ is much smaller than those involving single and double ruptures, and usually can be neglected.

\subsection{Conclusions}

We have formulated a general theoretical approach that provides a quantitative description of the detachment force distribution of a peptide from a lipid membrane. The proposed model assumes that detachment occurs, with certain probability $\left(w_{n}\right)$, along a few dominant diffusive pathways, characterized by three parameters $\left(\Delta U_{0 n}, \Delta x_{0 n}\right.$, and $\left.k_{0 n}\right)$. We have identified these pathways with last-rupture events involving one or two residues, in general, located at the end of the peptide. The values of the energetic $\left(\Delta U_{0 n}\right)$ and geometric $\left(\Delta x_{0 n}\right)$ parameters, which are residue and lipid species specific, can be derived from existing free energy profile studies, while the kinetic parameters $\left(k_{0 n}\right)$ and pathway weights $\left(w_{n}\right)$ should be treated as fitting parameters. As shown in the next sections, this new theoretical approach provides a consistent interpretation of relevant AFM dynamic force spectroscopy data. Although our model cannot predict the occurrence probability of a particular pathway in individual experiments, as shown in the next chapters, once the pathways have been identified and characterized, the rupture force histogram $P(F)$ can be matched by only using $w_{n}$ 's as fitting parameters, which is quite remarkable.

Our theoretical model is capable of reproducing accurately both retraction speed and 
non-monotonic force dependence of the rupture rate $k(F)$. Furthermore, it predicts that for moderate $v$ and intermediate $F$ values, $k(F)$ should exhibit catch-bond behavior, namely a decrease with increasing $F$, before continuing to increase again, as would normally expect. According to the model, the origin of the catch-bond mechanism in peptide-lipid membrane interactions is the stochastic mixing of individual detachment pathways, which do not convert or cross during rupture. This catch-bond mechanism is manifestly different from the commonly used "two-pathway" model [30] for ligand-receptor systems.

At last but not least, our theoretical model can also be used to calculate the intrinsic detachment (or off) rate, $k(0)=\sum_{n=1}^{N} w_{n} k_{0 n}$, of a peptide interacting with a lipid membrane. Clearly, the result should be independent (within margin of errors) of the retraction speed and, thus, can be regarded as a consistency test of the multiple pathway model for peptidelipid membrane detachment.

Finally, one should mention that, beside the detachment (last rupture) forces, the retraction force time series, $F(t)$, contains detailed information about the entire peptide-lipid membrane interaction process, including intermediate rupture events. In the framework of our theoretical approach, the latter may be regarded as detachment events of intermediate residues. However, such analysis is beyond the scope of this Thesis, and further work is needed to apply and test our theory for such intermediate rupture events. 


\section{Chapter 3}

\section{Forced detachment of the peptide SecA2-11 from zwitterionic and polar lipid bilayers ${ }^{1}$}

Here we demonstrate the effectiveness of the theoretical model described in the previous chapter by applying it to AFM-based single molecule force spectroscopy measurements of the peptide SecA2-11 interacting with (i) zwitterionic POPC lipid bilayer and (ii) polar E. coli lipid membrane.

SecA, a protein distributed between the cytosol and the cell membrane, is one of the major component of the general secretory system $[34,35]$. As a membrane-associated ATPase, SecA has to bind to the cell membrane in order to execute its role in precursor protein translocation. It has been shown experimentally that the binding of SecA to the membrane is accomplished through a short segment of 10 amino acids located at the Nterminus of the protein, SecA2-11, with primary structure LIKLLTKVFG. The AFM tips

\footnotetext{
${ }^{1}$ The content of this chapter is based on M. Utjesanovic et al, Scientific Reports 9, 451 (2019) [1], and T.R. Matin, M. Utjesanovic et al (2019)
} 


\begin{tabular}{ccc}
\hline \hline Residue & $\Delta U_{0}\left[k_{B} T\right]$ & $\Delta x_{o}[\mathrm{~nm}]$ \\
\hline $\mathrm{L}$ & 8.0 & 1.0 \\
$\mathrm{I}$ & 10.0 & 1.3 \\
$\mathrm{~S}$ & 2.0 & 0.7 \\
$\mathrm{R}$ & 8.2 & 1.3 \\
\hline \hline
\end{tabular}

Table 3.1: PMF model parameters describing the interaction of selected residues with POPC lipid bilayer. [26, 27]

used in the experiments were functionalized with SecA2-11 through flexible PEG linkers (with $\sim 10 \mathrm{~nm}$ contour length) [2].

The detachment force, $F$, of SecA2-11 (derived from SecA) from each of the two lipid membranes mentioned above was measured in a series of high precision (sub-pN) AFM force spectroscopy experiments [10], performed under a variety of conditions. The constructed experimental $P(F)$ histograms showed significant variation from experiment to experiment, even for the same peptide-lipid membrane system. Attempts to model $P(F)$ by using the theory for a single detachment pathway failed. We attribute this failure to the complexity of the peptide-lipid membrane interactions. Here we show that in fact $P(F)$ can be modeled in a consistent way by assuming that peptide-lipid membrane detachment involves a small number (usually $N=3$ or 4 ) of dominant pathways of single and double rupture events. The modeling strategy is as follows. First, one identifies the residues at the end of the peptide that are most likely to rupture last. Next, the corresponding values of the PMF parameters, $\Delta U_{0 n}$ and $\Delta x_{0 n}$, are identified from previous MD simulation studies, $[26,27]$ which reconstructed the PMF of various residues interacting with POPC bilayers. These values are listed in Table 3.1. Finally, the intrinsic detachment rates $k_{0 n}$ (kinetic parameters) and occurrence probabilities $w_{n}$ (weight coefficients) for each participating detachment pathway $(n=1, \ldots, N)$ are determined by fitting the experimental $P(F)$ histograms using Eqs. (2.10) and (2.11). As a general rule, because the PMF parameters 
$\left(\Delta U_{0 n}\right.$ and $\left.\Delta x_{0 n}\right)$ are determined by the nature of the peptide and lipid membrane, their values should not be changed during the fitting process. At the same time, the rates $k_{0 n}$ may depend slightly on the experimental conditions (e.g., retraction speed $v$, AFM cantilever tip), but not as much as the weight factors $w_{n}$, which may change considerably from experiment to experiment. While the convergence of $w_{n}$ to a peptide-lipid membrane specific value may require a prohibitively large number of measurements, the parameters identifying the individual pathways (i.e., $\Delta U_{0 n}, \Delta x_{0 n}$, and $k_{0 n}$ ) should be obtainable from a relatively small number of single molecule experiments.

\subsection{Detachment of SecA2-11 from zwitterionic POPC bi- layer}

In the reminder of this chapter we demonstrate the viability of the multiple detachment pathways model described above by applying it to interpret the rupture force histograms, $P(F)$, and the corresponding force dependent dissociation rates, $k(F)$, of SecA2-11 interacting with a zwitterionic POPC lipid bilayer and a polar E. coli cell membrane.

\subsection{1 $P(F)$ : AFM-tip dependence}

The experimental $P(F)$ histogram of SecA2-11 interacting with POPC lipid bilayer (for $v=100 \mathrm{~nm} / \mathrm{s}$ ) shown in Fig. 3.1a can be fitted well by assuming $N=4$ detachment pathways. Two of these $(n=1,2)$ are identified as single ruptures involving the last two residues, $\mathrm{L}$ and I, of the peptide. The other two pathways $(n=3,4)$ correspond to double rupture processes involving the same residues. The corresponding PMF parameters are

listed in Table 3.1, while the fitting parameters, $k_{0 n}$ and $w_{n}$, are listed in Fig. 3.1a. Note 


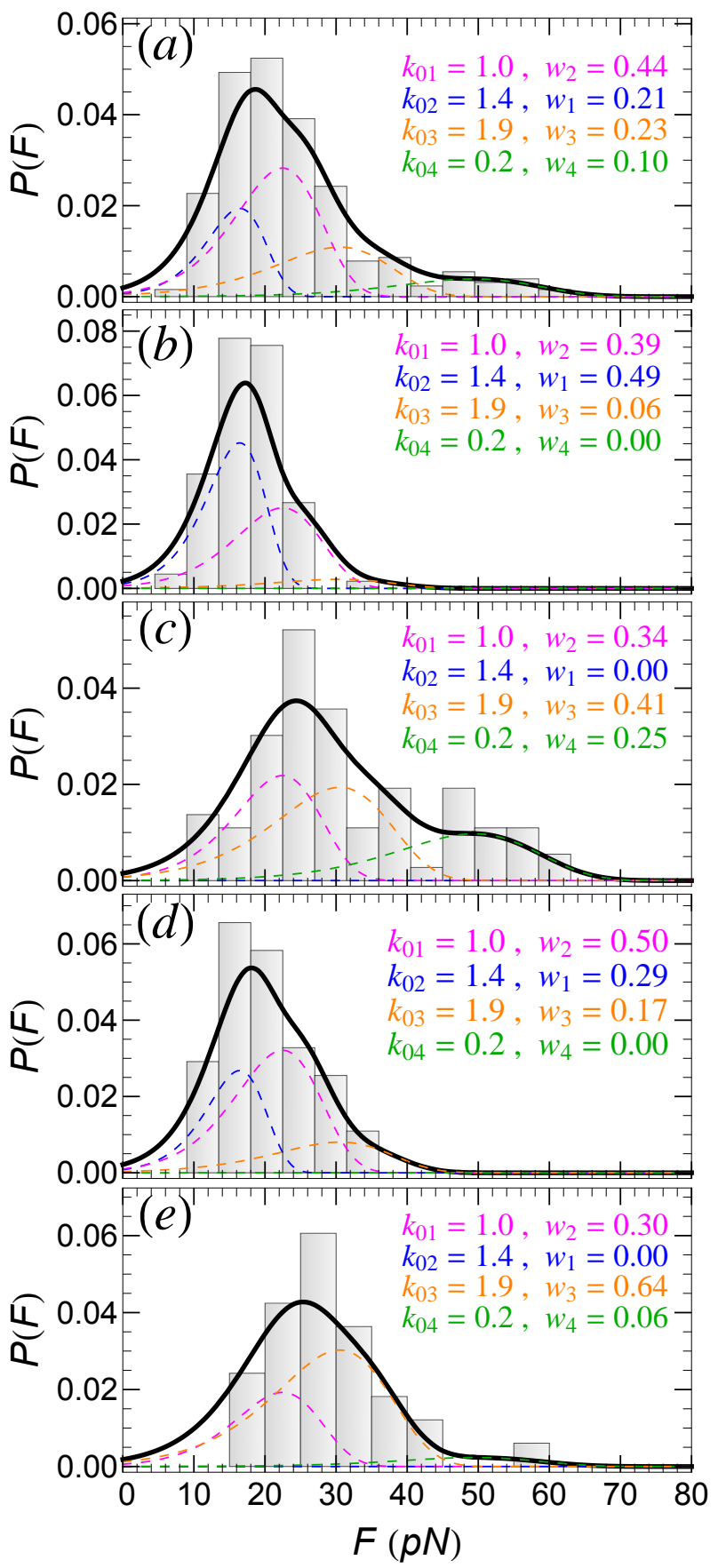

Figure 3.1: Data shown is from (a) all AFM tips, and (b)-(e) four individual AFM tips. In each case, $P(F)$ can be fitted well by using four detachment pathways (solid-thick curves). Contributions to $P(F)$ from the $n$-th $(n=1, \ldots, 4)$ detachment pathways are shown as colored dashed curves. The corresponding intrinsic rupture rates, $k_{0 n}$, and weights, $w_{n}$, are also listed. 
that, in this case, the single rupture events $(65 \%)$ are more prevalent than double ruptures (33\%). Also, the predominant four pathways account for $98 \%$ of $P(F)$; the remaining $2 \%$ correspond to other pathways that occur only seldom and, thus, can be neglected.

The $P(F)$ histogram in Fig. 3.1a contains data from several AFM retraction experiments, performed with different, but otherwise identically prepared, AFM tips. Reflecting the stochastic nature of single molecule experiments, the $P(F)$ histograms for individual tips, shown in Fig. 3.1b-e, have completely different shapes. However, all these different histograms can be well fitted assuming the same $N=4$ detachment pathways identified above, but with different weight factors $w_{n}$. This means that, in otherwise identically prepared samples, certain detachment pathways may be favored against others. While, in general, one cannot predict the prevalence of a particular pathway in individual experiments, it is still remarkable that $P(F)$ can be reproduced by only using $w_{n}$ 's as actual fitting parameters. Thus, the detachment pathways, identified through three parameters (i.e., $\Delta U_{0 n}, \Delta x_{0 n}$, and $k_{0 n}$ ), can be regarded as fingerprints of a specific peptide-lipid membrane system.

\subsection{2 $P(F)$ : retraction speed, $v$, dependence}

The experimental $P(F)$ histogram of SecA2-11 interacting with POPC lipid bilayer for six different retractions speeds are shown in Fig. 3.2. In all these cases too, $P(F)$ can be fitted well by assuming the same four detachment pathways identified in the previous section, and by simply adjusting the corresponding weight factors $w_{n}$. It appears that for the lowest retraction speeds, $v=30$ and $50 \mathrm{~nm} / \mathrm{s}$, the double rupture pathways are the dominant ones, accounting respectively for $85 \%$ and $62 \%$ of $P(F)$. For higher speeds, the situation is reversed, in favor of the single ruputre pathways. 

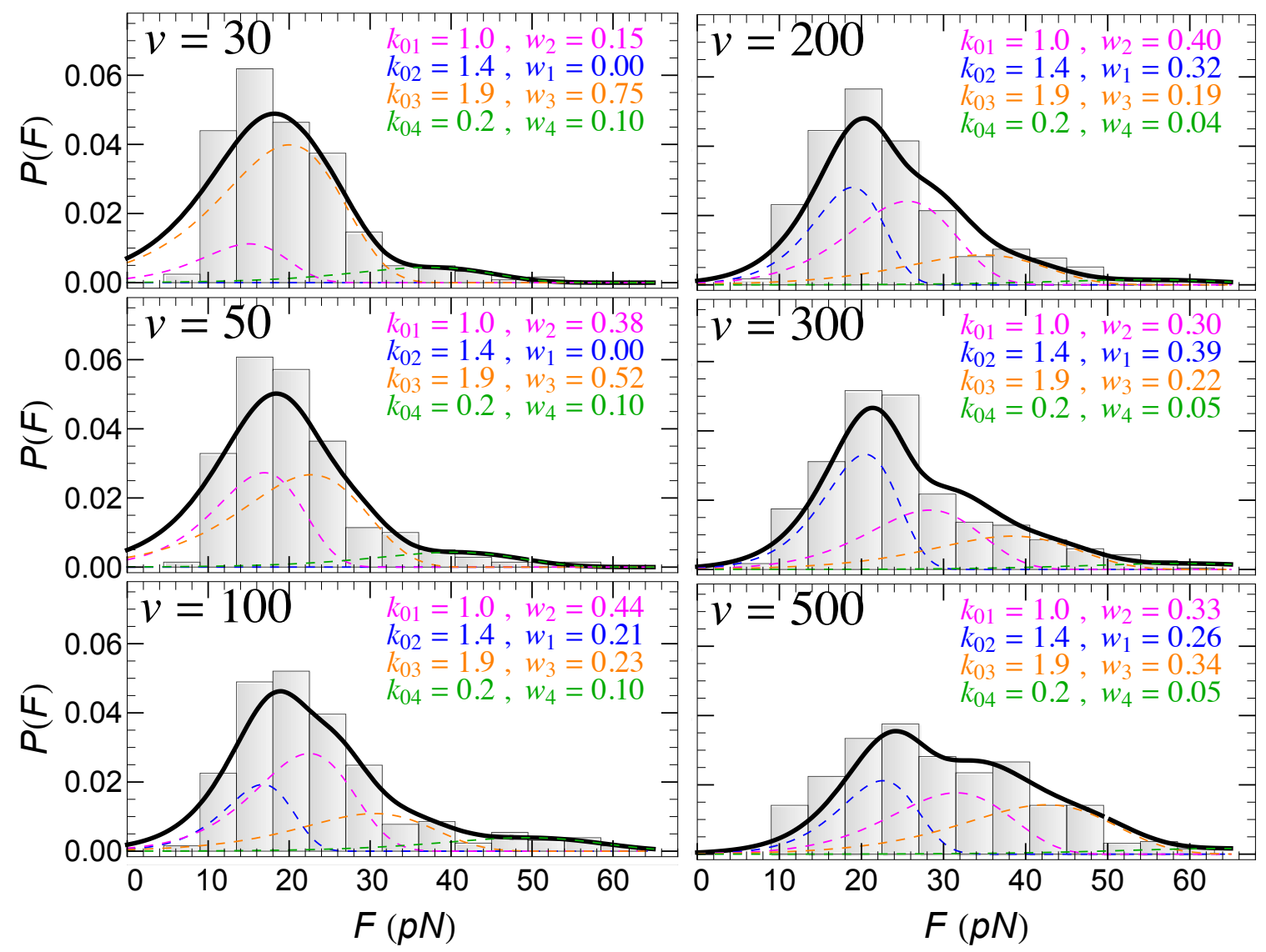

Figure 3.2: In each case, $P(F)$ can be fitted well by using four detachment pathways (solidthick curves). Contributions to $P(F)$ from the $n$-th $(n=1, \ldots, 4)$ detachment pathways are shown as colored dashed curves. The corresponding intrinsic rupture rates, $k_{0 n}$, and weights, $w_{n}$, are listed. 


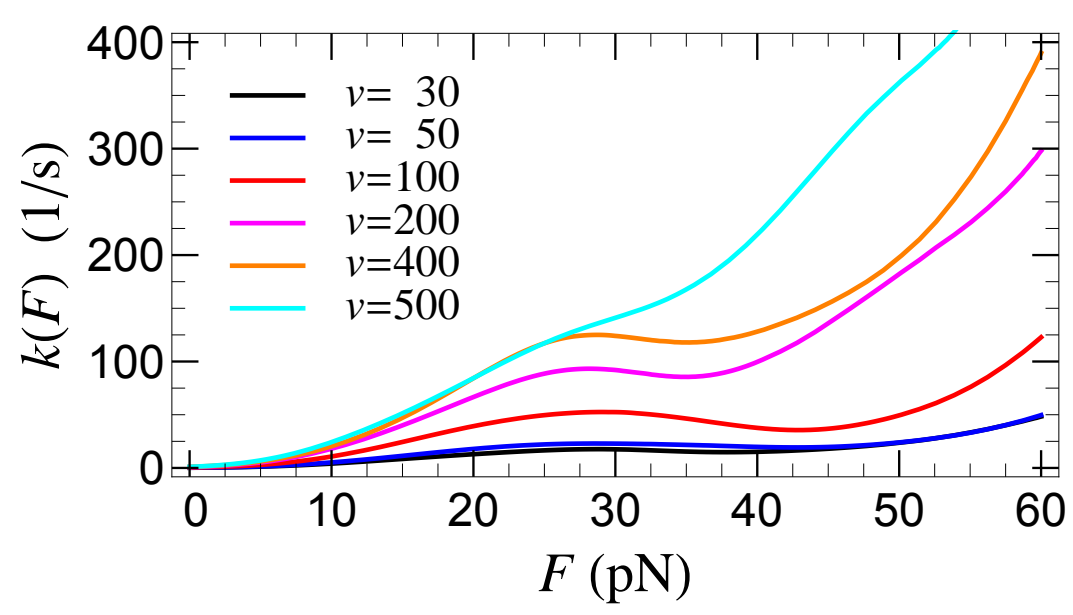

Figure 3.3: Force dependent detachment rate, $k(F)$, was determined from Eq. (2.9), for the listed retraction speeds (in $\mathrm{nm} / \mathrm{s}$ ) of the AFM tip.

\subsection{3 $k(F)$ : retraction speed, $v$, dependence}

As already mentioned, the strong $v$ dependence of $k(F)$, determined from the experimental $P(F)$ by means of Eq. (2.9), and shown in Fig. 3.3, clearly demonstrates that peptidelipid bilayer detachment cannot be described by a single detachment pathway, thus lending support to our multiple pathways method. While the individual rates, $k_{n}(F)$, for each detachment pathway are monotonically increasing with $F$, the non-monotonic behavior of $k(F)$, given by Eq. (2.12), is due to the $F$-dependent contributions, through the coefficients $\alpha_{n}(F)$, of the individual pathways. The situation is illustrated, for $v=100 \mathrm{~nm} / \mathrm{s}$, in Fig. 3.4.

It is remarkable that, for intermediate forces, the stochastic mixing between the different pathways may lead to "catch-bond" behavior [28-30], where counterintuitively the detachment rate decreases with the increase of the applied force (Fig. 3.4b). Note that the origin of the catch-bond in this case is quite different from the "two-pathway" model, according to which catch-bond behavior in ligand-receptor systems comes about through pathway switching (inner conversion) [30]. In our case, individual peptide-lipid detach- 


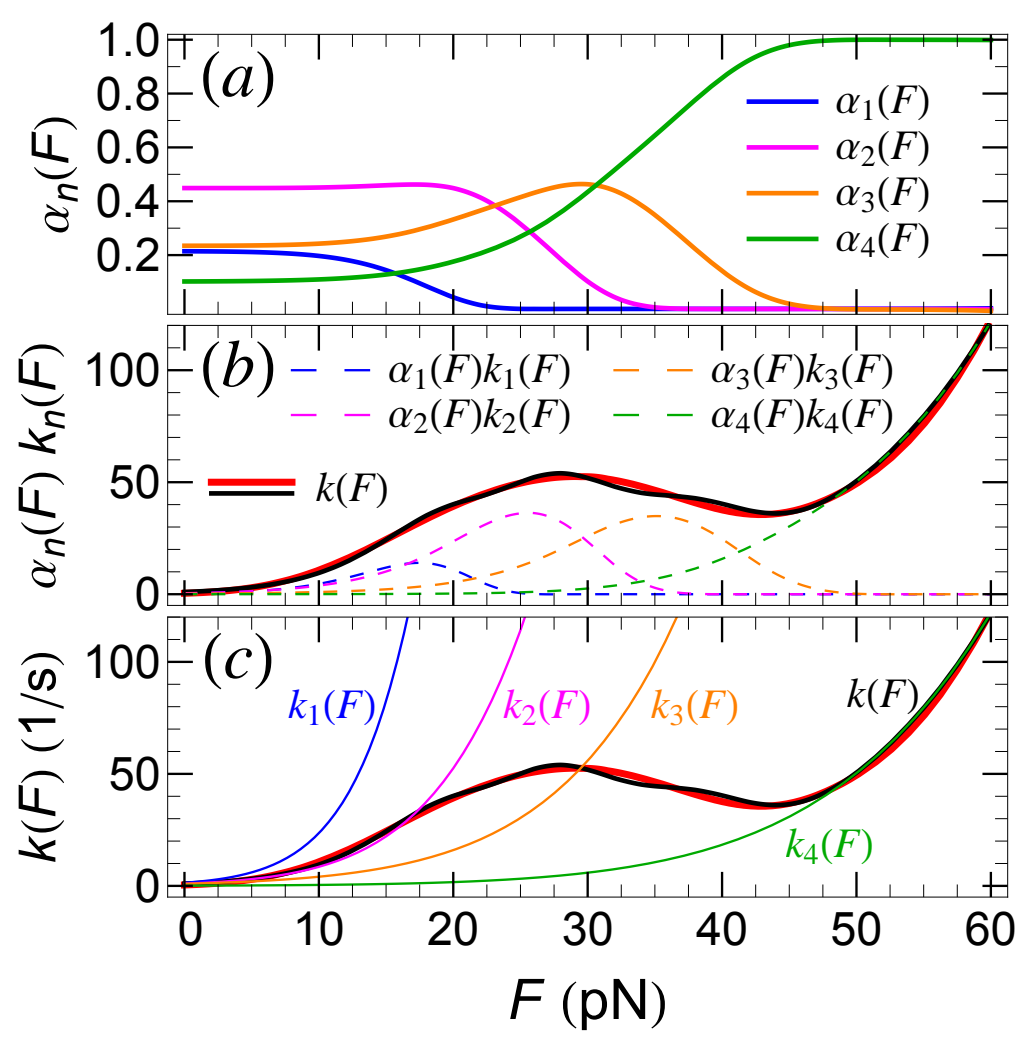

Figure 3.4: (a) Force dependent weight factors for each detachment pathway. (b) $k(F)$ derived using $P(F)$ from experiment (thick, solid red curve) and our theoretical modeling (solid black curve). The weighted contributions of individual pathways to $k(F)$, i.e., $\alpha_{n}(F) k_{n}(F), n=1, \ldots, 4$, are also shown (dashed curves). (c) Pathway specific, force dependent rupture rates, $k_{n}(F)$. For reference, the experimental and theoretical $k(F)$ are also shown as in (b). 
ment events occur, with a given probability, along well defined pathways, which do not cross or convert during rupture. The force dependent coefficients $\alpha_{n}(F)$ in Eq. (2.6) are all positive but their derivatives, $d \alpha_{n} / d F$, may become negative for certain force values which gives rise to catch bond behavior (see Fig. 3.4a). Thus, we are dealing with a new catch-bond mechanism that is specific to peptide-lipid membrane dissociation (or similar molecular processes) [1].

\subsection{Detachment of SecA2-11 from polar $E$. coli membrane}

Next, we consider the detachment of SecA2-11 from the biologically relevant $E$. coli cell membrane, which is a mixture of anionic POPG (20 mol \%) and zwitterionic POPE (70 mol \%) lipids, and cardiolipin (10 mol \%). We begin with comparing the detachment force distributions, $P(F)$, for the two lipid membranes, i.e., POPC and E. Coli.

\subsection{1 $P(F)$ : dependence on the type of lipid membrane}

Figure 3.5 compares the rupture force distributions, $P(F)$, of SecA2-11 interacting with a supported (a) POPC lipid bilayer, and (b) patch of E. coli membrane. In both cases, the theoretical models (black lines) used to fit the experimental $P(F)$ histograms (red lines) contain four dominant detachment pathways, corresponding to two single $(n=1,2)$ and two double $(n=3,4)$ rupture events. The two single ruptures are assigned to the last two residues (i.e., $\mathrm{L}$ and I) at the N-terminus of SecA2-11. For the corresponding activation energies $\left(\Delta U_{0 n}\right)$ and lengths $\left(\Delta x_{0 n}\right)$ we have used the same values as for the POPC bilayer (see Table 3.1). The justification for this choice is twofold: (i) there is no PMF data for residues in E. coli membrane, and (ii) the fraction of the zwitterionic lipids in E. coli is much larger 


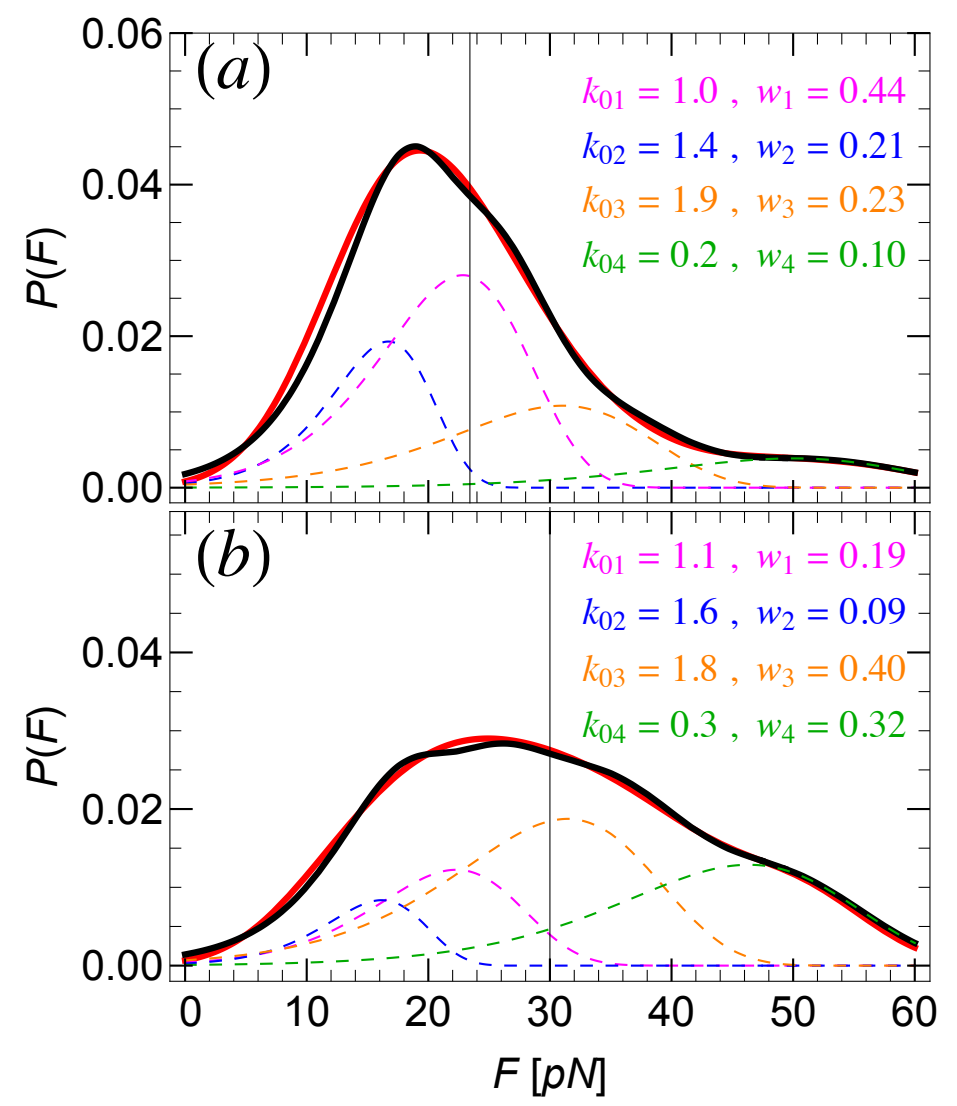

Figure 3.5: Experimental (solid red line) and theoretical model (solid black line) detachment force distribution $P(F)$ for SecA2-11 in (a) POPC lipid bilayer and (b) E. coli cell membrane. The theoretical $P(F)$ required four model force distributions (colored dashed lines) corresponding to distinct dissociation pathways, as described in the text. The intrinsic rupture rates, $k_{0 n}$, and weights, $w_{n},(n=1, \ldots, 4)$, are also listed. The vertical lines indicate the mean rupture force. 
than that of charged lipids. While for individual pathways the intrinsic rates, $k_{0 n}$, show only weak membrane species dependence, the weight coefficients, $w_{n}$, vary considerably, likely due to the complex nature of peptide-lipid membrane interactions.

$P(F)$ is significantly broader in the case of the anionic E. coli membrane (mean rupture force $\bar{F}=30 \mathrm{pN})$ than for the zwitterionic PC bilayer $(\bar{F}=23 \mathrm{pN})$. Indeed, the kurtosis of $P(F)$ is much larger for $\mathrm{PC}(\beta=5.2)$ than for E. coli $(\beta=2.2)$, meaning that the force distribution for PC (E. coli) is sharper (broader) than a normal distribution (for which $\beta=3$ ). The reason for this is that double rupture events (with higher rupture forces) are more abundant for E. coli $(>70 \%)$ than for POPC $(<35 \%)$. In addition to direct electrostatic effects, such dramatic increase of double ruptures in E. coli membrane could also be due to the presence of secondary structure elements that the peptide adopts when bound to E. coli polar lipid. Helical structure, if present, could give extra rigidity to the peptide that would favor the (almost) simultaneous dissociation of two residues.

\subsection{2 $P(F)$ : retraction speed, $v$, dependence}

Figure 3.6 shows the experimental $P(F)$ (red lines) for SecA2-11 interacting with $E$. coli membrane for four different retractions speeds, $v=50,100,200,300 \mathrm{~nm} / \mathrm{s}$. It is remarkable that, in spite of the strong $v$-dependence, the rupture force distribution can be fit well (black lines) by using the same four dissociation pathways identified above. Note that, apart from minor adjustment of the intrinsic dissociation rates for the double ruptures, the fitting simply requires the recalculation of the weight factors $w_{n}$. For retraction speeds $v<300 \mathrm{~nm} / \mathrm{s}$, the contribution to $P(F)$ of the double rupture pathways dominate $(>50 \%)$. However, at the highest pulling speed $v=300 \mathrm{~nm} / \mathrm{s}$, this contribution decreased below 40\%, and single ruptures dominate. A plausible explanation is that the higher the retraction speed the higher 


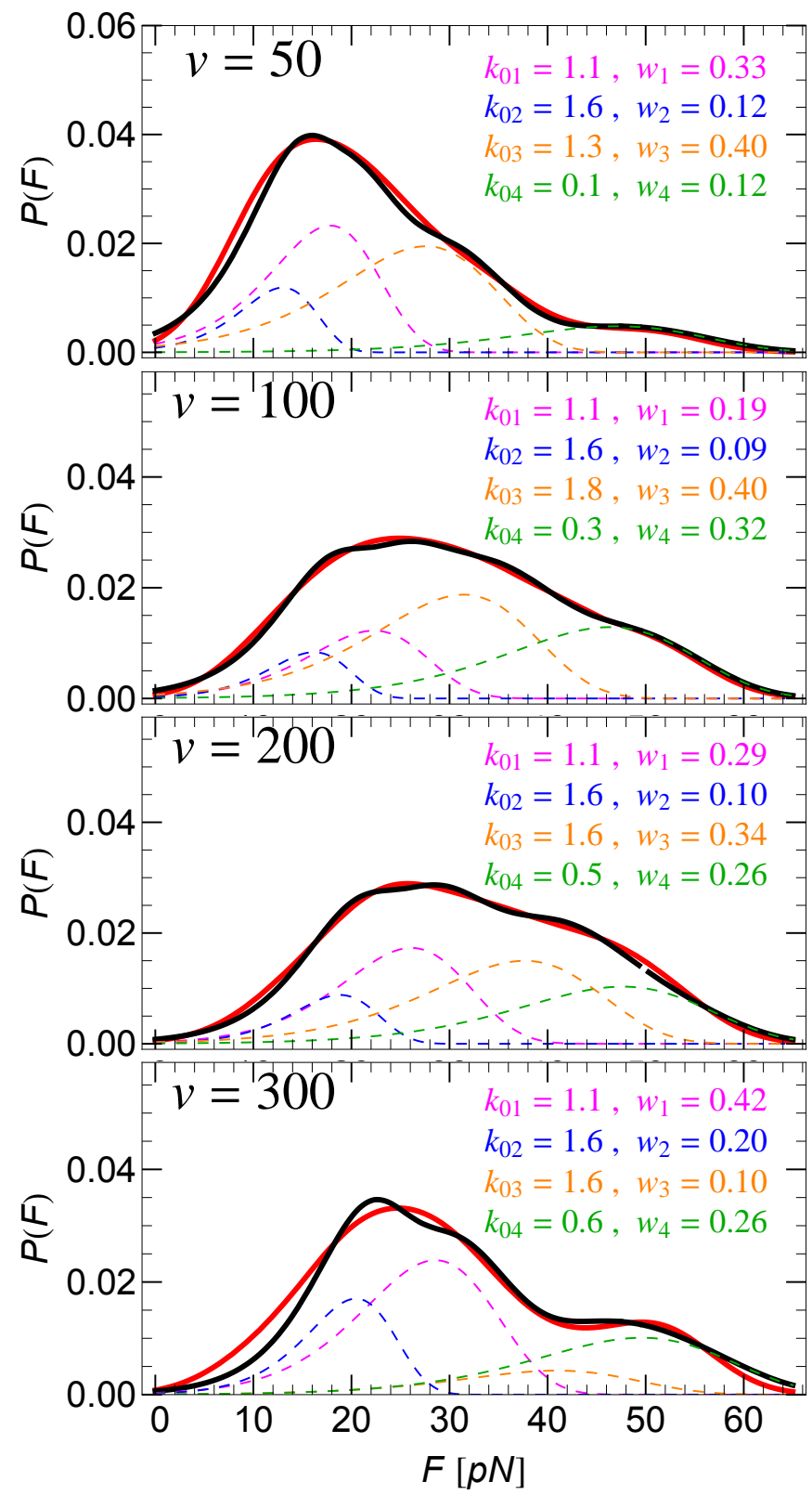

Figure 3.6: The experimental results (solid red curves) are matched well be the theoretical model (solid black curves) by using four detachment pathways. Contributions to $P(F)$ from the individual pathways are shown as colored dashed curves; the corresponding intrinsic rupture rates, $k_{0 n}$, and weights, $w_{n}$, (with $n=1 \ldots 4$ ) are also listed. 


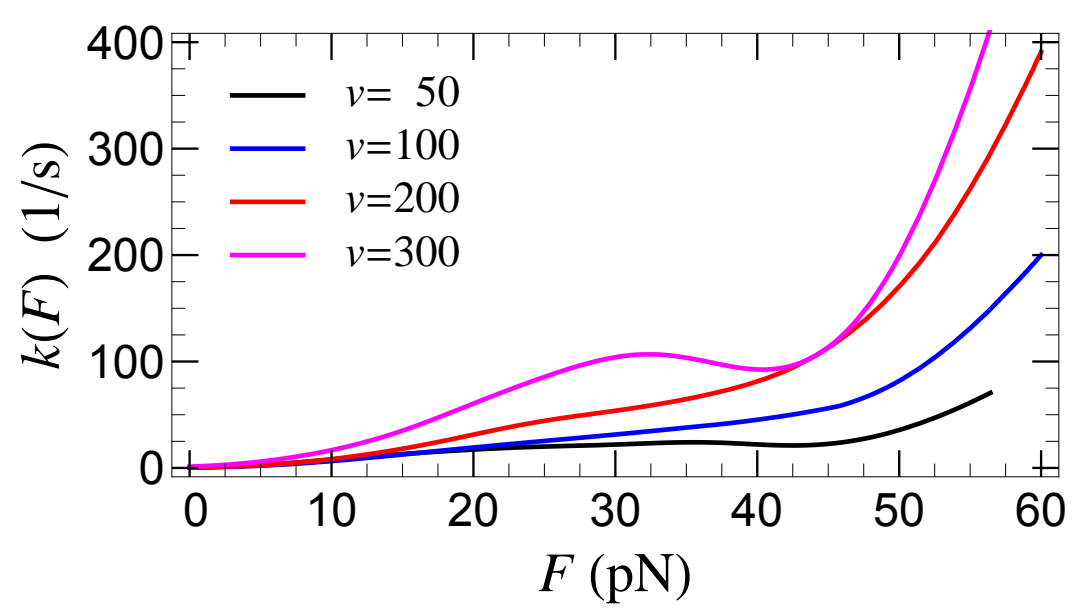

Figure 3.7: Force dependent detachment rate, $k(F)$, of SecA2-11 from E. coli membrane, for the listed retraction speeds (in $\mathrm{nm} / \mathrm{s}$ ) of the AFM tip.

the probability that the peptide completely unfolds before detachment from the membrane, thus significantly reducing the occurrence of double ruptures.

\subsection{3 $k(F)$ : retraction speed, $v$, dependence}

In case of SecA2-11 interacting with E. coli membrane, the force and retraction speed dependent detachment rate, $k(F)$, can be calculated (in model independent fashion) from $P(F)$, by using Eq. (2.9). The results are shown in Fig. 3.7. Again, the strong $v$-dependence and non-monotonic behavior of $k(F)$ is a clear indication that peptide-lipid bilayer detachment is a multi-pathway process [1]. Note that while the individual single-detachment rates, $k_{n}(F)$, increase monotonically with $F$, the effective $k(F)$ is non-monotonic, as it can be inferred from Eq. 2.11, in which the coefficients $\alpha_{n}(F)$ are force dependent.

Furthermore, $k(F)$ may exhibit catch bond behavior [1], where counter-intuitively the dissociation rate decreases with increasing applied force, i.e., $d k(F) / d F<0$, [28-30]. A catch bond region is manifest in the $v=300 \mathrm{~nm} / \mathrm{s}$ curve at $F \approx 35 \mathrm{pN}$ (Fig. 3.7). 


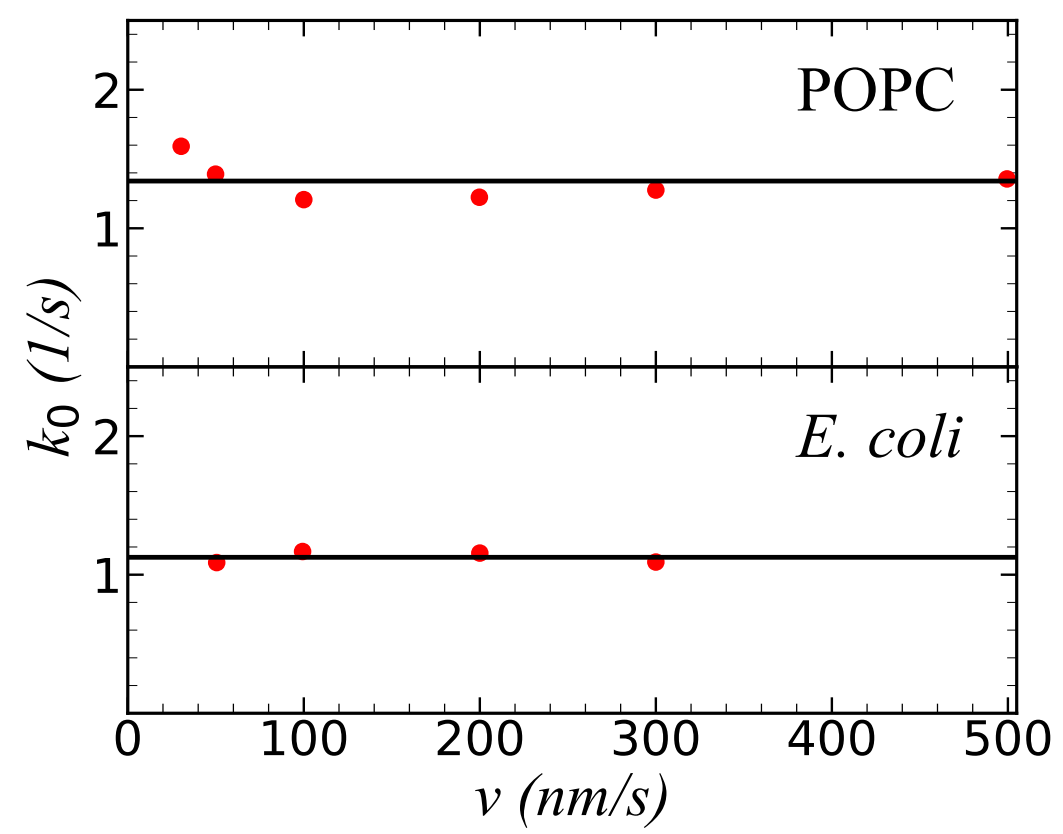

Figure 3.8: The intrinsic detachment rate, $k_{0}=k(F=0)$ (red filled-circles) of SecA211 interacting with POPC bilayer (top panel) and E. coli membrane (bottom panel) show independence on AFM retraction speed, $v$. Horizontal black lines represent the mean value $\left\langle k_{0}\right\rangle$.

\subsection{Dissociation rate of SecA2-11 from POPC and E. coli lipid membranes}

In spite of the significant $v$-dependence of $k(F)$, one finds that the intrinsic (dissociation) off-rate of SecA2-11 from both POPCE. coli membrane, $k_{0}=k(0)=\sum_{n=1}^{4} w_{n} k_{0 n}$, is essentially independent of $v$, as shown in Fig. 3.8. The off-rate we obtained here, $k_{0}=1.13 \pm 0.04 \mathrm{~s}^{-1}$ (mean \pm standard deviation), is about $12 \%$ lower than that for the same peptide interacting with a PC bilayer $\left(k_{0}=1.27 \pm 0.09 \mathrm{~s}^{-1}\right)$ [1]. These, normally expected but non-trivial results, lend further support to our multiple detachment pathways model. 


\subsection{Conclusions}

We have formulated and validated a general theoretical approach that provides a quantitative description of the detachment force distribution of a peptide (e.g., SecA2-11) from a lipid membrane (e.g., POPC, E. coli). The proposed model assumes that detachment occurs, with certain probability $\left(w_{n}\right)$, along a few dominant diffusive pathways, characterized by three parameters $\left(\Delta U_{0 n}, \Delta x_{0 n}\right.$, and $\left.k_{0 n}\right)$. We have identified these pathways with lastrupture events involving one or two residues, in general, located at the end of the peptide. The values of the energetic $\left(\Delta U_{0 n}\right)$ and geometric $\left(\Delta x_{0 n}\right)$ parameters, which are residue and lipid species specific, can be derived from existing free energy profile studies, while the kinetic parameters $\left(k_{0 n}\right)$ and pathway weights $\left(w_{n}\right)$ are used as fitting parameters. This new theoretical approach allowed for a consistent interpretation of the considered experimental data. Interestingly, even for the same peptide-lipid membrane system, the occurrence frequency of different dominant detachment pathways (measured through $w_{n}$ ) showed significant AFM tip dependence, although these tips were prepared identically. However, even if one cannot predict the occurrence probability of a particular pathway in individual experiments, it is quite remarkable that, once the pathways have been identified and characterized, the rupture force histogram $P(F)$ can be matched by only using $w_{n}$ 's as fitting parameters.

Our theoretical model also reproduced accurately both retraction speed and non-monotonic force dependence of the rupture rate $k(F)$. For moderate $v$ and intermediate $F$ values, $k(F)$ exhibits catch-bond behavior, namely a decrease with increasing $F$, before continuing to increase again, as normally expected. According to our model, the origin of the catchbond mechanism in peptide-lipid membrane interactions is the stochastic mixing of individual detachment pathways, which do not convert or cross during rupture. This catch-bond 
mechanism is manifestly different from the commonly used "two-pathway" model [30] for ligand-receptor systems.

Finally, the calculated intrinsic dissociation (or off) rate, $k(0)=\sum_{n=1}^{N} w_{n} k_{0 n}$, of the peptide SecA2-11 from the considered lipid membranes, was independent (within margin of errors) of the retraction speed, which is quite remarkable if we take into account that the pathway weights $w_{n}$ are $v$-dependent while the individual rates $k_{0 n}$ are not. 


\section{Chapter 4}

\section{MD simulations of SecA2-11 interacting with zwitterionic POPC and charged POPG bilayers ${ }^{2}$}

While AFM based single molecule force spectroscopy is very efficient in measuring, with high accuracy, the strength of the interaction between peptide and lipid membrane, unfortunately, it cannot provide any direct information about the conformational details of the system. Therefore, to gain insight into the conformational dynamics of SecA2-11 during its interaction with lipid bilayers, we have used long time scale, all-atom MD simulations. To explore the limiting cases, we have built two membrane systems, one comprised of $100 \%$ anionic PG lipid, and the other $100 \%$ zwitterionic PC lipid. In principle, a linear combination of the obtained results can be used to approximate the behavior of the E. coli polar lipid mixture.

Previous circular dichroism (CD) spectrum measurements showed that SecA2-11 has

\footnotetext{
${ }^{2}$ The content of this chapter is based on T.R. Matin, M. Utjesanovic et al, PNAS (2019)
} 
no to little secondary structure in POPC bilayer, whereas it acquires a measurable fraction of helical structure in E. coli. Due to the lipid composition of the E. coli membrane, it is reasonable to assume that the presence of the helical secondary structure in SecA2-11 is facilitated by its interaction with the anionic PG lipids. Along this line of reasoning, one expects that the helical content of SecA2-11 should be even higher in a pure POPG bilayer. Indeed, our MD results show that SecA2-11 acquires elements of helical structure upon binding to charged POPG lipid bilayer, but not to POPC. Importantly, the mean penetration depths of the individual amino acids, as determined via simulations, were in good overall agreement with previously published power saturation electron paramagnetic resonance (EPR) results emerging from full length SecA [36]. The agreement between the results for SecA2-11 and the full-length SecA implies that indeed the extreme N-terminus of SecA acts as an independent structural entity through which the protein binds to the cell membrane. At the same time, this important result lends further support to our theoretical and computational approach to describe peptide-lipid membrane interactions.

\subsection{Methods}

\subsubsection{Building the systems}

A completely extended, atomistic model of the peptide SecA2-11 (LIKLLTKVFG-C) was created in the Molefacture plugin in VMD [37]. To closely mimic the experimental system, in which the peptide is covalently bonded through a Cys residue to a PEG linker attached to a functionalized AFM tip, a Cys residue was added to the C-terminus of SecA2-11 [2]. Two model systems were then built by placing a copy of the peptide parallel to the surface of 
fully solvated (and pre-equilibrated) lipid bilayers made respectively of zwitterionic POPC (system PC), and anionic POPG lipids (system PG). Each bilayer had 64 lipids per leaflet, i.e., a total of 128 lipids. The POPC bilayer was built using the Membrane plugin in VMD [37] and solvated with 5895 TIP3P water molecules [38], while the POPG bilayer was built in CHARMM-GUI $[39,40]$ and solvated with 4971 TIP3P waters. Using the Autoionize plugin in VMD, system PC (PG) was neutralized by adding $2 \mathrm{Cl}^{-}\left(2 \mathrm{Cl}^{-}\right.$and $128 \mathrm{~K}^{+}$) counter ions. Prior to adding the peptide, each bilayer was first equilibrated for $\sim 100 \mathrm{~ns}$ in the NApT ensemble. During this time, the dynamics of lipids was monitored (i.e., by following the bilayer thickness and the acyl chain order parameter) to make sure that the systems reached equilibrium (Fig. 4.1). Finally, system PC (PG) comprised a total of 35030 (31490) atoms, and (after equilibration) occupied an orthorhombic unit cell with dimensions $72 \times 64 \times 72 \AA^{3}\left(72 \times 64 \times 66 \AA^{3}\right)$.

\subsubsection{MD simulations}

All simulations were performed using NAMD2.12 [41] and the CHARMM36 force field [42-44]. The MD equations of motion were integrated with a multiple time stepping scheme [45] with the r-RESPA algorithm [46]. The used time steps were, respectively, 1,2 and 4 fs for bonded, short-range (van der Waals) and long-range (electrostatic) interactions. Van der Waals interactions were cut off at $12 \AA$ with a smooth switching function starting at $10 \AA$ A Long-range electrostatic interactions were computed with the Particle Mesh Ewald (PME) method [47], with a grid spacing of $1 \AA$. After proper minimization and several stages of equilibration, $0.5 \mu$ s long production runs of equilibrium MD in the $N P T$ ensemble were performed with both PC and PG. The temperature was kept constant at $T=300 \mathrm{~K}$ by using a Langevin thermostat [48] with a damping coefficient of $1 \mathrm{ps}^{-1}$. 

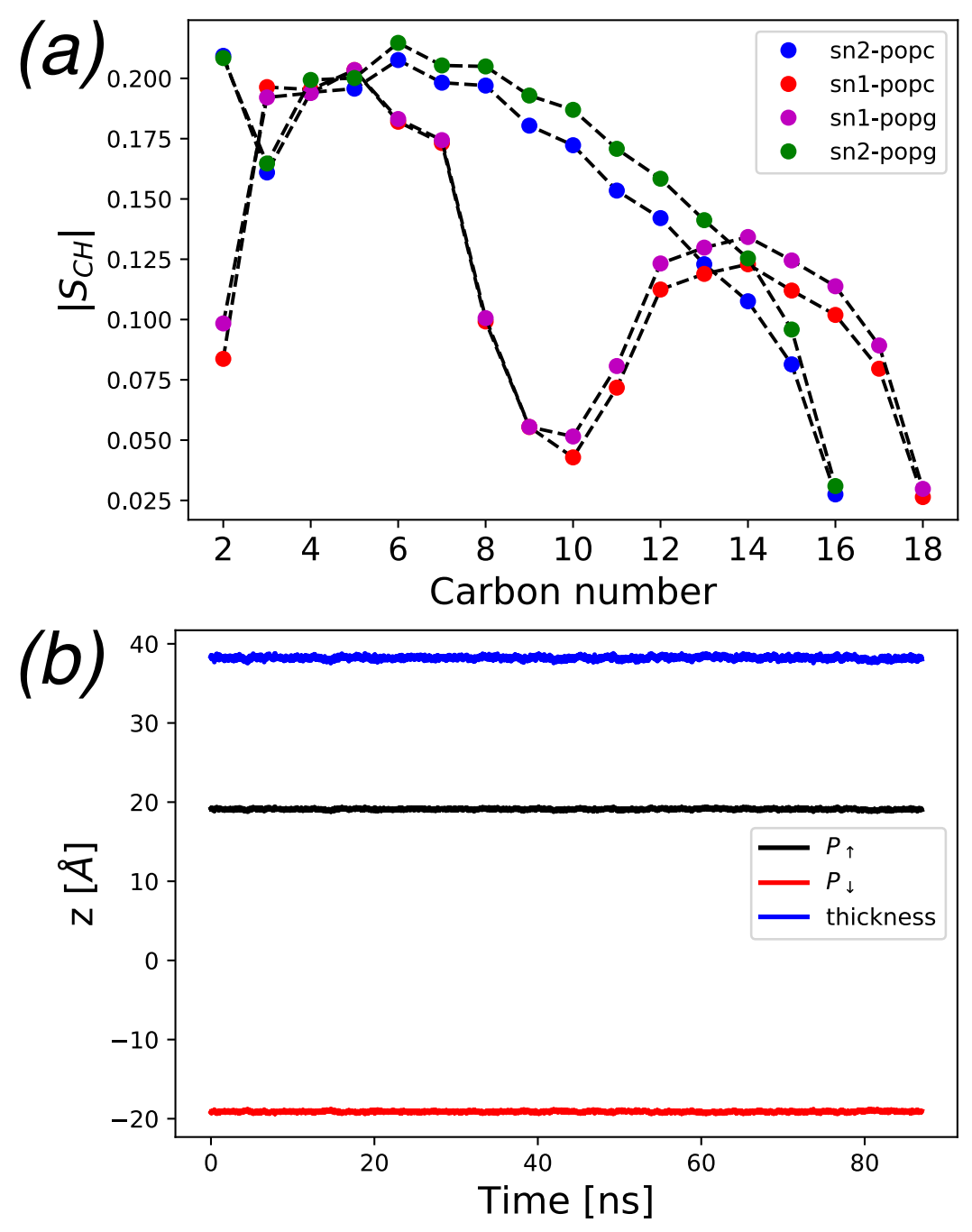

Figure 4.1: (a) Acyl chain order parameters (sn1 and sn2) for systems PC (blue and red) and PG (magenta and green), averaged over the last $80 \mathrm{~ns}$ of equilibrium run. (b) Time evolution of the z-coordinate (direction normal to the plane of the bilayer, and origin at the center of the membrane) of the center of mass of the phosphate atoms in the upper (black) and lower (red) leaflets, respectively. The time evolution of the thickness of system PC is shown in blue. On the scale of the figure, the results for system PG are indistinguishable from PC and, thus, are omitted. 
The pressure was maintained at normal value $(P=1 \mathrm{~atm})$ by employing the Nosé-Hoover Langevin piston barostat [49] with period of $100 \mathrm{fs}$ and decay time of $50 \mathrm{fs}$.

In order to mimic the supported lipid membranes used in the AFM experiments, the phosphorous (P) atoms from the lower leaflet of the phospholipid bilayer in both PC and PG systems were harmonically restrained $\left(k=5 \mathrm{kcal} / \mathrm{mol} / \AA^{2}\right)$ during the MD simulations, thus preventing the drift of the membrane along the $z$-direction, normal to its plane. We have verified that the applied restrains did not alter the dynamics and behavior of the systems, as no noticeable change of the bilayer thickness, the density profile of P (and other lipid) atoms, or the acyl chain order parameter were observed. The MD simulations were carried out on several GPU accelerated workstations equiped with 36 Intel Xeon CPUs cores, with a performance of around $36 \mathrm{~ns} /$ day. All analyses (i.e., relative penetration depth, helical content, solvent-accessible-surface-area) of the MD simulations were performed with MDAnalysis [50,51], MDTraj [52], and VMD [37].

\subsection{Results and Discussion}

The two $0.5 \mu \mathrm{s}$ long MD trajectories of PC and PG provide insight, at atomistic level, in the partitioning of SecA2-11 from solution to POPC and POPG lipid bilayer, respectively. In both simulations the peptide inserts spontaneously into the bilayers on a time scale of $\sim 100 \mathrm{~ns}$.

\subsubsection{Membrane penetration depth of SecA2-11}

To quantify the degree of penetration into the membrane of the peptide, the position of its center of mass $(\mathrm{COM})$ relative to the surface of the membrane, $\Delta z(t)$, was calculated. The 


\begin{tabular}{|c|c|c|c|}
\hline System & $E_{\text {es }}[\mathrm{kcal} / \mathrm{mol}]$ & SASA $\left[\AA^{2}\right]$ & $R_{g}[\AA]$ \\
\hline \hline PC & $-258.35 \pm 82.14$ & $1712.55 \pm 57.39$ & $8.46 \pm 0.47$ \\
PG & $-294.05 \pm 102.04$ & $1645.59 \pm 57.56$ & $7.28 \pm 0.65$ \\
\hline
\end{tabular}

Table 4.1: Electrostatic interaction energy $\left(E_{\mathrm{es}}\right)$, solvent accessible surface area (SASA) and radius of gyration $\left(R_{g}\right)$ of SecA2-11 in PC and PG.

surface of the bilayer was defined by the mean position of the $\mathrm{P}$ atoms in the considered leaflet. The time evolution of the peptide-membrane separation, $\Delta z(t)$, in both PC and PG, is plotted in Fig. 4.2. As expected, the results show that the COM of the positively charged peptide penetrates deeper in the neutral POPC than in the anionic POPG bilayer.

After 300 ns, both systems appear to be well equilibrated, with the COM of SecA211 fluctuating about a mean level located slightly below (above) the surface of the POPC (POPG) bilayer. Overall, SecA2-11 in POPG is located mostly in the headgroup region, while in POPC it penetrates deeper into the membrane. This result seems to be consistent with the fact that the electrostatic interaction between the peptide and membrane is stronger in PG than in PC (see Table 4.1).

Furthermore, due to the stronger peptide-membrane interaction, SecA2-11 has a more compact configuration in POPG than in POPC (Fig 4.2a-d), consistent with its smaller solvent-accessible-surface-area (SASA) and radius of gyration (see Table 4.1).

A more precise quantitative description of the penetration of the peptide in a membrane is to specify $\Delta z$ for each residue (identified, e.g., through the $\mathrm{C}_{\alpha}$ atom or the COM of its sidechain) with respect to the surface of the membrane. The $\Delta z$ values for the ten SecA211 residues in both POPC and POPG, obtained from the MD simulations, are shown in Fig. 4.3a. Note that the values of $\Delta z$ depend only slightly whether the residues are identified through their $\mathrm{C}_{\alpha}$ atom or their sidechain's COM. Also, while the $\Delta z$ values for the five residues from the C-terminus are similar in both POPC and POPG, these values for the five 

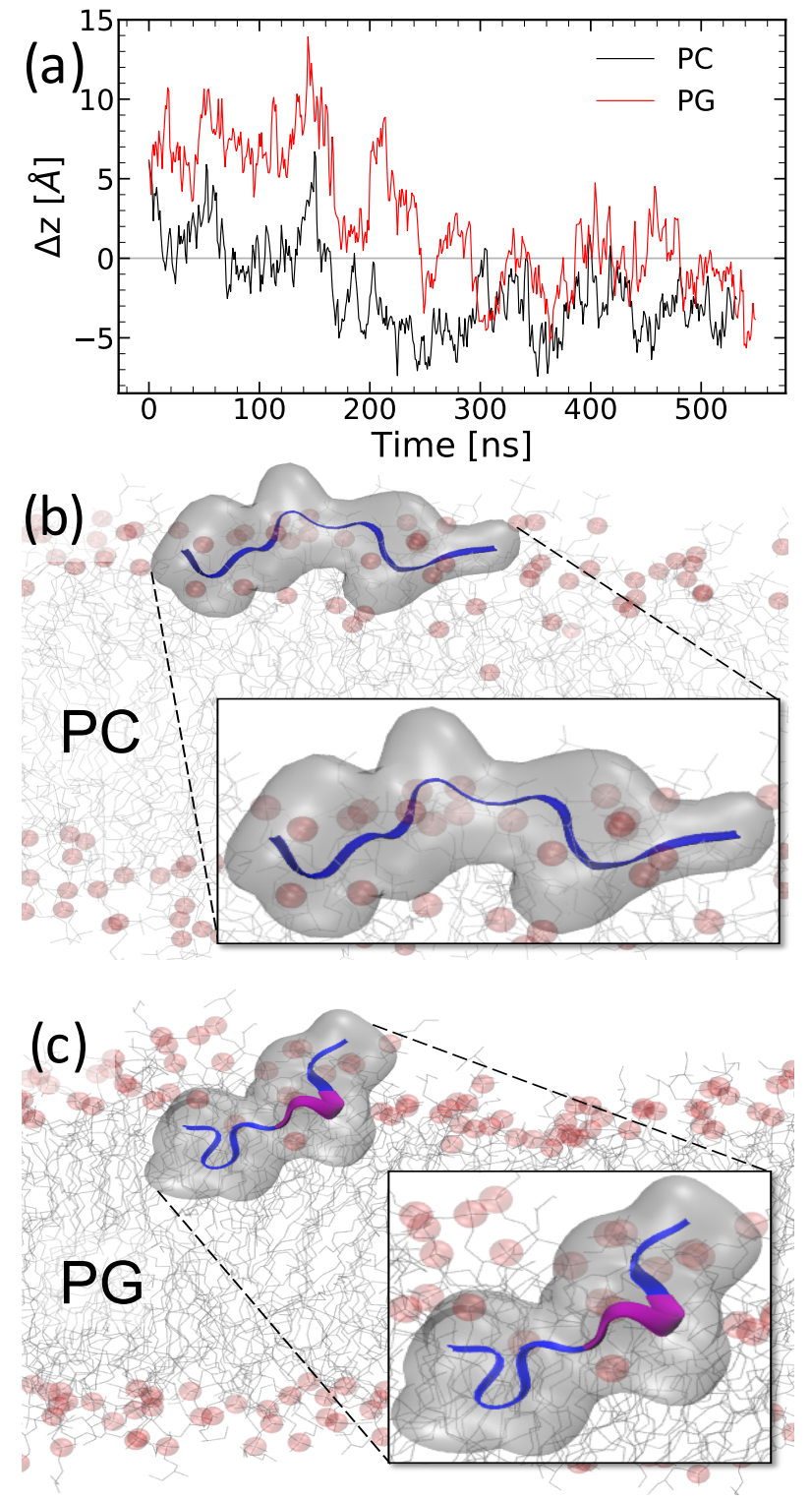

Figure 4.2: (a) Time evolution of the position, along the z-axis, of the COM of SecA2-11 relative to the surface of the membrane (defined by the mean position of the $\mathrm{P}$ atoms, which was set as origin, $z=0$ ) in both PC (black line) and PG (red line). Representative snapshots of (b) PC and (c) PG taken respectively at 350 and $360 \mathrm{~ns}$. The backbone of the peptide is shown in cartoon representation and is colored according to secondary structure (magenta for $3_{10}$ helix and blue for random coil). The transparent surface representation indicates the spatial extent of SecA2-11. The P atoms in the headgroup of the lipids are shown as red spheres and, for clarity, water molecules were omitted. All snapshots were rendered using VMD [37]. 


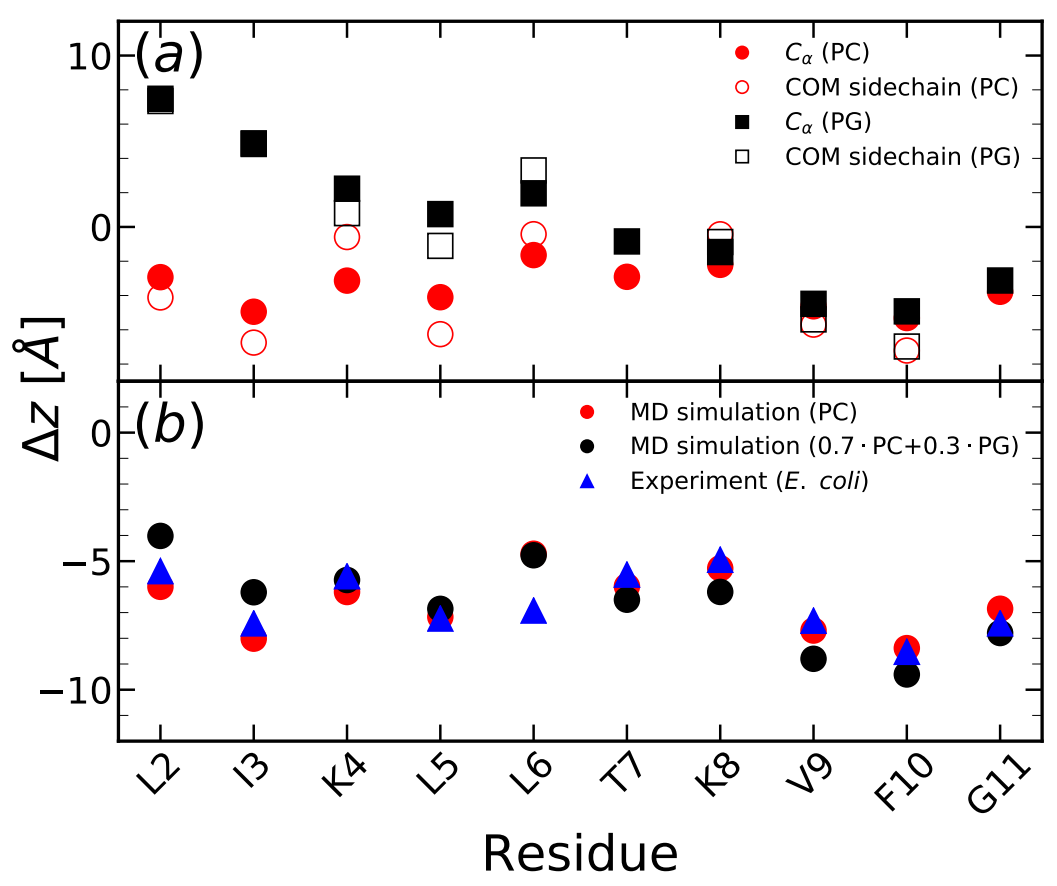

Figure 4.3: (a) Average penetration depth $(\Delta z)$ of the SecA2-11, per residue and averaged over simulation time. $\Delta z$ is calculated with respect to the phosphate centers in upper leaflet of PC (red) and PG (black), expressed in terms of the position of $C_{\alpha}$ atom (filled markers) and side chain COM (hollow markers). (b) Comparison between the EPR experimental [36] (triangles) and MD simulation (circles) results for $\Delta z$ of the SecA2-11 residues. The $-3 \AA$ overall shift applied to the MD data is comparable to the uncertainty in defining the position of the membrane surface. The MD data correspond to SecA2-11 in PC (red circles) and an $E$. coli membrane mimic (black circles), determined as a weighted average of the PC $(70 \%)$ and PG (30\%) results. 
residues from the $\mathrm{N}$-terminus are noticeably larger in POPG than in POPC. This result is consistent with the time evolution of the distance of the COM of SecA2-11 from the surface of the two bilayers shown in Fig. 4.2 and discussed above.

In a recent experimental study of the interaction between the full protein SecA with the E. coli membrane [36], the penetration depth of the ten residues of SecA2-11, with respect to the surface of the membrane defined by the phosphate group, was measured. As shown in Fig. $4.3 \mathrm{~b}$, the measured $\Delta z$ values appear to be in good agreement with our MD simulation results for PC and, also, for an $E$. coli membrane mimic for which $\Delta z$ was calculated as a weighted average for PC (70\%) and PG (30\%). Note that the MD results were subject to a $-3 \AA$ overall shift, which is comparable to the size of the phosphate group and, thus, to the uncertainty in defining the position of the membrane surface.

\subsubsection{Helical content of SecA2-11}

To estimate SecA20-11's helical content in POPC (POPG), we have used the DSSP algorithm [53] for secondary structure assignment. In POPC, just like in solution, SecA2-11 is an extended random coil, with no secondary structure. Conversely, in POPG, due to the stronger peptide-membrane interaction, SecA2-11 acquires intermittent, partial (one turn) $3_{10}$ helical structure. The three residues involved in the formation of the $3_{10}$ helical turn are $\mathrm{Lys}^{4}, \mathrm{Leu}^{5}$ and $\mathrm{Leu}^{6}$. The time series of the fraction of residues that form $3_{10}$ helical structure are shown in Fig. 4.4. Our MD simulation results are in semi-quantitative agreement with the experimental CD spectrum measurements, according to which SecA2-11 has no to little secondary structure in POPC, while it acquires a measurable fraction of helical structure in E. coli membrane, most likely due to its POPG lipid content. 


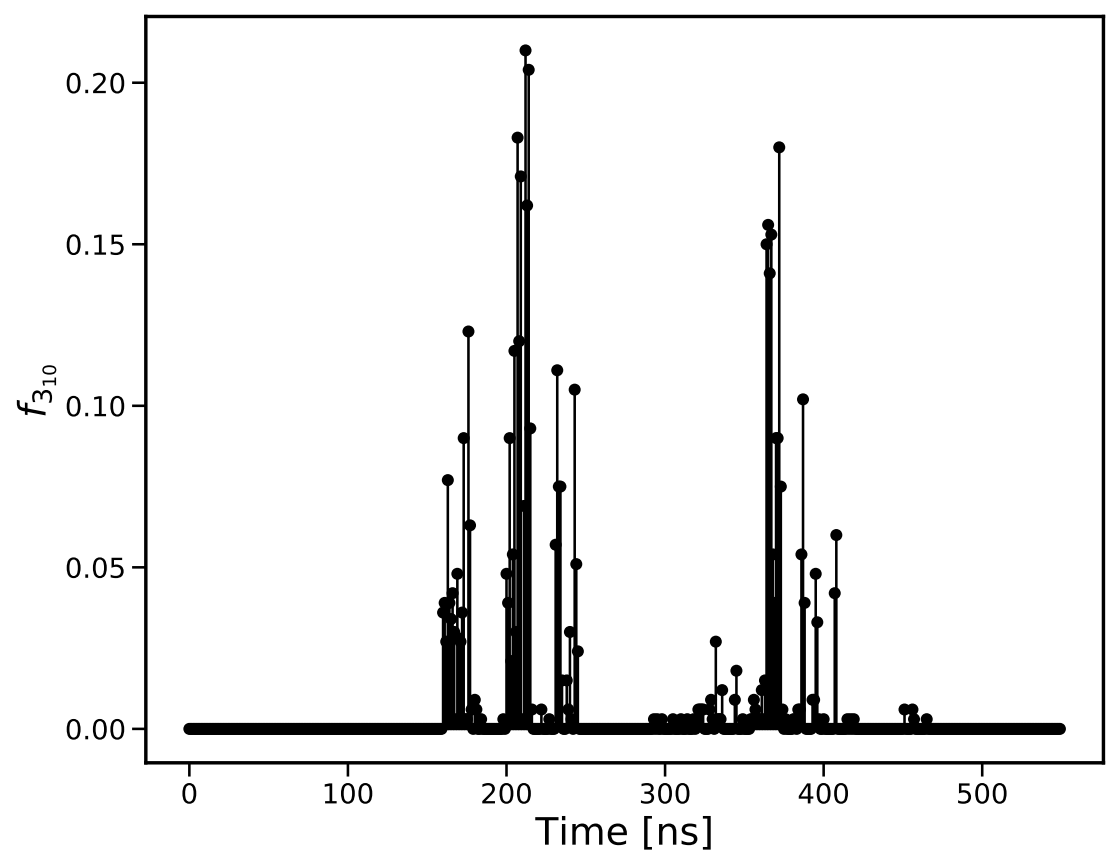

Figure 4.4: Result shown are averaged over $1 \mathrm{~ns}$ of simulation time, i.e. 100 frames. $f_{3_{10}}$ represents fraction of residues that participate in $3_{10}$ helix. 


\subsection{Conclusions}

The good agreement between the MD simulations of SecA2-11 and EPR results using full length SecA lends support not only to the validity of the MD simulation methodology, but also to the significance of the extreme $\mathrm{N}$-terminus of SecA as an adhesion element to the membrane, and further, to the notion that the $\mathrm{N}$-terminal region of SecA acts independently from the remainder of the the protein [54].

In addition, the qualitative agreement between the MD simulations of SecA2-11 and the $\mathrm{CD}$ experiments confirmed that it is more likely for the peptide to form (structurally more robust) helical structure in a polar E. coli membrane than in a zwitterionic POPC lipid bilayer. This may explain why there are more double-residue rupture events (resulting in broader detachment force, $P(F)$, distribution) in the case of the detachment of SecA2-11 from E. coli membrane compared to POPC bilayer. 


\section{Chapter 5}

\section{Conformational dynamics and energetics of melittin and its diastereomer interacting with POPC and POPG lipid bilayers: a molecular dynamics study}

\subsection{Introduction}

It was argued in Ch. 3 that significantly larger number of double-residue ruptures of SecA211 in E. coli membrane than in zwitterionic POPC is due to the presence of helical structure. In general, secondary structure elements provide extra rigidity to the peptide, thus favoring the simultaneous dissociation of two (i.e., double rupture) or more residues. One may assume that in such situation the secondary structure of the peptide survives the dissociation process. By contrast, when secondary structure dissolves during the retraction of the peptide, before its full detachments from the membrane, the occurrence of single ruptures are much more likely than double (or multiple) ruptures. 
In order to test this hypothesis one needs to study and compare the interaction between a lipid membrane with two conformers of the same peptide, i.e., peptides with same primary, but different secondary structures. Ideal candidates for this purpose are wild type melittin (MWT) and its diastereomer, $\mathrm{D}-\mathrm{V}^{5,8}, \mathrm{I}^{17}, \mathrm{~K}^{21}$-melittin (MD4). In aqueous solution, at low salt concentration and neutral pH, MWT has low helical content [55-59]. However, when it binds spontaneously to the surface of a zwitterionic or anionic phospholipid bilayer, MWT assumes a linear $\alpha$-helical conformation [60-62]. Unlike MWT, MD4 binds only to charged (e.g., POPG, E. coli) membranes and has reduced helical content even on a lipid bilayer interface. The latter is due to the four enantiomers (i.e., D-amino acid substitutions of $\mathrm{V}^{5,8}, \mathrm{I}^{17}$ and $\mathrm{K}^{21}$ ) that act as "helix-breakers".

Melittin is an extensively studied peptide and a major component of the honey bee venom Apis mellifera [63]. The amphiphilic nature of melittin, due to its hydrophobic Nand hydrophilic C-terminal regions, plays a major role in its interaction with lipid membranes [64-67] (Fig. 5.1). The relatively strong binding of melititn to both zwitterionic and polar lipid membranes can be attributed to a combination of (at least) four factors [68], namely the (i) electrostatic attraction between the positively charged residues to the hydrophilic lipid head group, (ii) insertion of the hydrophobic helical segment into the lipid bilayer, (iii) peptide folding, and (iv) peptide aggregation in the lipid phase.

The axis of melittin's $\alpha$-helix can be oriented either parallel (i.e., inactive conformation) or perpendicular (i.e., active conformation associated to pore formation) to the plane of the lipid bilayer [69]. The reorientation of melittin from its inactive to active conformation is crucial for pore formation and can only occur for sufficiently high protein-lipid $(\mathrm{P} / \mathrm{L})$ ratios $[66,67,70-72]$. In fact, the lytic (antimicrobial) character of melittin manifests through the formation of tetrameric pores across membranes [73], which leads to a leakage of the 
intracellular content [74-79].

Computer simulations provide detailed, microscopic level description of peptide-lipid membrane systems on length and time scales that are inaccessible to experimental measurements. Most of the existing MD studies of melittin mainly focus on the formation and stability of toroidal pores across the membrane $[59,66,67,71,80-83]$. Melittin mediated pore formation is a relatively slow process that takes place on a larger than millisecond time scale $[84,85]$. Previous MD studies have overcame this limitation either by placing multiple regularly arranged melittin copies inside of a bilayer as a part of initial structure [86-89] or by using coarse-grained simulations [90].

Our goal here is to investigate the conformational dynamics and energetics of MWT and MD4 interacting with zwitterionic POPC and anionic POPG lipid bilayer by using all-atom MD simulations. In particular, we seek to (i) assess the strength of peptide-lipid membrane interaction through the degree of penetration of MWT and MD4 into POPC and POPG lipid bilayers; (ii) estimate the degree of helical (secondary structure) content of MWT and MD4 in POPC and POPG; and (iii) determine the free energy profile (potential of mean force or PMF) of MWT and MD4 with parallel orientation to the surface of POPC and POPG, respectively.

While there are a few MD studies of MWT oriented parallel to the surface of a lipid membrane $[84,85,91]$ to the best of our knowledge, there are no such studies for MD4. Unlike in previous studies $[84,85,91]$, in our MD simulations we have followed the conformational dynamics of melittin (both MWT and MD4) by starting from a completely folded configuration. Thus, by resorting to (only) hundreds of ns long free MD simulations, we were able to readily identify whether, under the considered conditions, the peptide had a stable secondary structure (i.e., when it remained folded) or not (i.e., when it unfolded). 

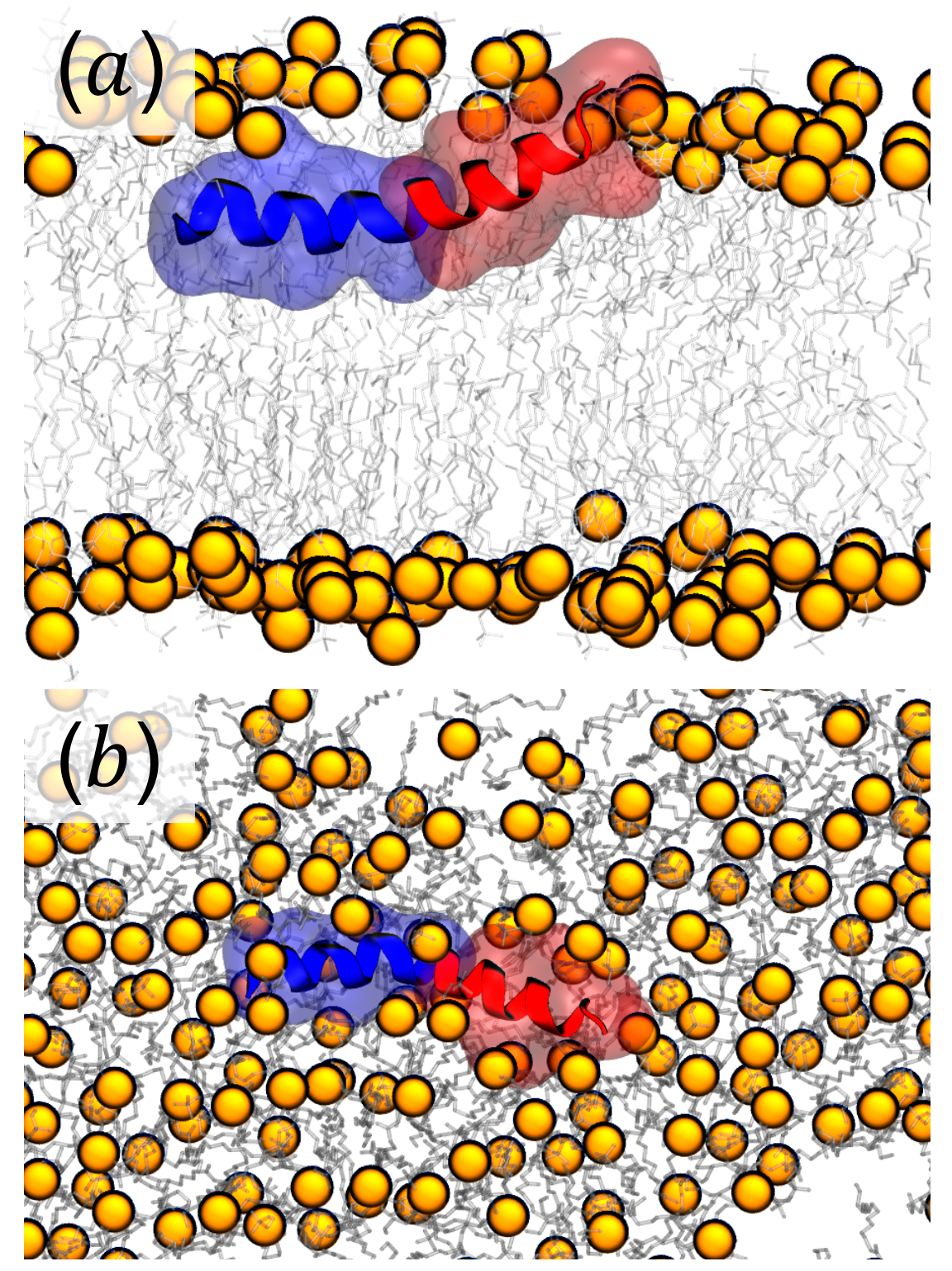

Figure 5.1: (a) Side and (b) top view of MWT in POPC lipid bilayer. The hydrophobic segment formed by residues 1-14 (blue) penetrate deeper in the bilayer than the hydrophilic segment comprising residues 15-26 (red). MWT is shown in cartoon representation; the transparent surface indicates its spatial extent. The folded, helical conformation of the peptide persisted throughout the simulation. The P-atoms of the lipids are shown as orange VDW spheres. The images were rendered using VMD. 
By contrast, when starting from an unfolded conformation, one cannot rule out the possible existence of a stable folded state if the peptide does not fold by the end of the longest MD simulation that one can afford.

From our MD simulations we have found that (i) both MWT and MD4 are mostly unstructured in solution (with $0.1 \mathrm{M}$ salt concentration); (ii) MWT binds to both POPC and POPG lipid bilayers, keeping most of its helical content; and (iii) MD4 binds only to the POPG bilayer and loses most of its helical content, in agreement with previous experimental results $[3,68,92,93]$. Also, we have corroborated and further quantified these results by determining and comparing the potential of mean force (PMF), as a function of peptide-lipid membrane separation, for MWT and MD4 interacting with both POPC and POPG bilayers. In particular, the obtained PMFs allowed us to estimate the binding energy of both MWT and MD4 (in their inactive conformation) to each of the two lipid bilayers. Moreover, in each case, we were able to established a direct correlation between the obtained binding energy and the mean number of hydrogen bonds involved.

\subsection{Methods}

\subsubsection{Building the systems}

\section{Peptides}

The crystal structure of fully-folded melittin was obtained from the Protein Data Bank (PDB code 2MLT, chain A). The sequence (primary structure) of the 26 residue long melittin is $\mathrm{H}_{3} \mathrm{~N}^{*}$-GIGAVLK${ }^{*}$ VLTTGLP-ALISWIK ${ }^{*} \mathrm{R}^{*} \mathrm{~K}^{*} \mathrm{R}^{*} \mathrm{QQ}-\mathrm{C}-\mathrm{CONH}_{2}$, where the charged residues are marked by “*”. Melittin has a net charge of $+6 e$ due to the protonated N- 


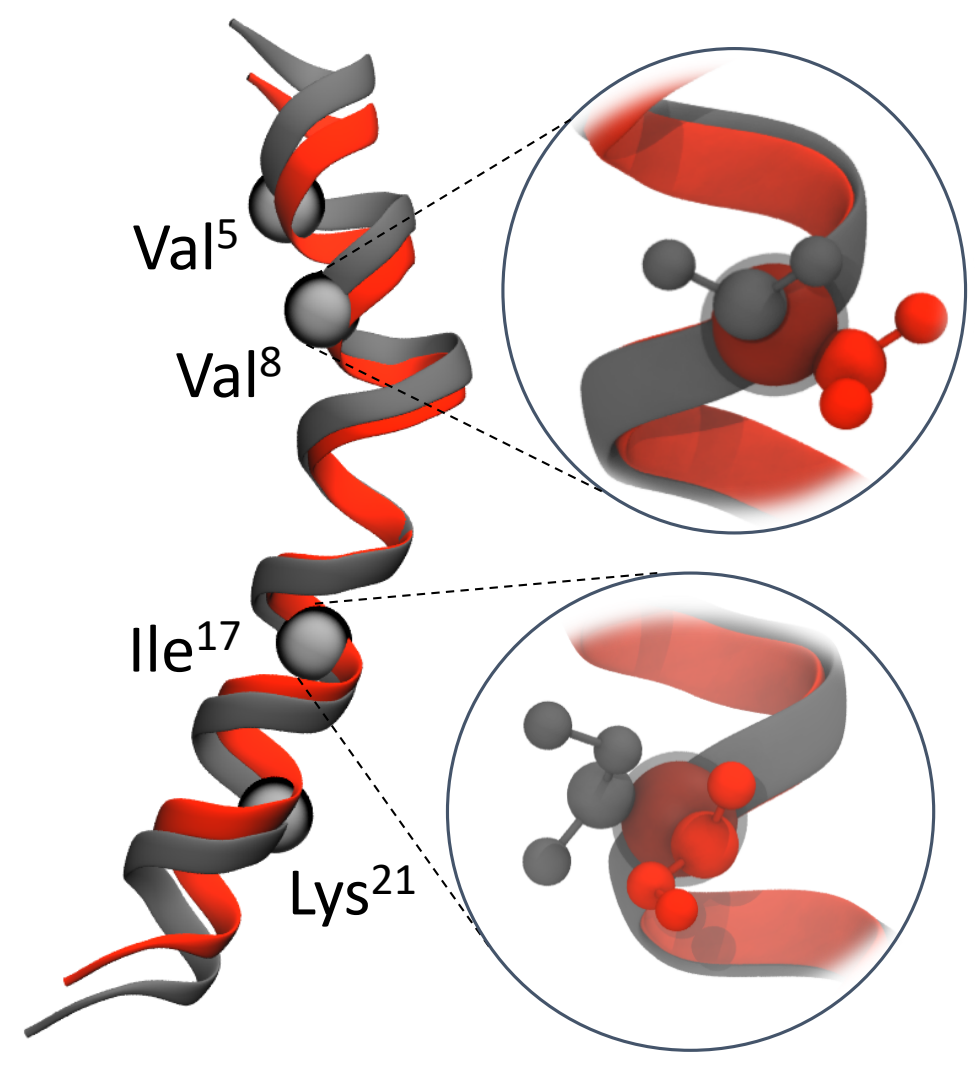

Figure 5.2: Initial structures of MWT and MD4 peptides. Aligned backbones (coil representation) of MWT (grey) and MD4 (red) show initially folded structure of peptides. We also show $C_{\alpha}$ atoms of $\mathrm{Val}^{5}, \mathrm{Val}^{8}, \mathrm{Ile}^{17}$ and $\mathrm{Lys}^{21}$ (grey vdW sphere) for reference. By zooming in $\mathrm{Val}^{8}$ and $\mathrm{Ile}^{17}$, the difference between the sidechains (CPK representation) of L(grey) and D-enantiomers (red) can be observed. All snapshots were created in VMD [37].

terminal and the 5 charged residues. Furthermore, a cysteine residue was added to the C-terminus of both MWT and MD4, in order to closely mimic the experimental system in which the peptide is covalently bonded through a Cys residue to a PEG linker attached to a functionalized AFM tip [2]. MD4 was built by employing SwissSideChain parameters $[94,95]$ in Chimera [96], where $\mathrm{Val}^{5,8}, \mathrm{Ile}^{17}$ and $\mathrm{Lys}^{21}$ residues of MWT were replaced with their D-enantiomers. Finally, by aligning the backbones MWT's and MD4's, we compare the initial structures of peptides in Fig. 5.2. 


\section{MWT/MD4 in aqueous solution}

Single copy of MWT (MD4) was solvated with 6548 (6631) TIP3P [38] water molecules. $12 \mathrm{~K}^{+}$and $18 \mathrm{Cl}^{-}$ions were added to each system to create a $0.1 \mathrm{M}$ neutral solutions that mimic physiological conditions. Each system occupied a periodic cubic cell of $60 \AA$ side.

\section{MWT/MD4 in zwitterionic POPC and anionic POPG lipid bilayer}

POPC and POPG bilayers, solvated with TIP3P water molecules [38], were generated in Membrane Plugin in VMD [37] and CHARMM-GUI [39, 97, 98], respectively. Peptides were placed parallel to the equilibrated bilayer surface, at distance of $\sim 1 \mathrm{~nm}$ from phosphate centers. To improve sampling, two copies of a same peptide were used, one on each side of membrane (Fig. 5.3).

\subsubsection{MD simulations}

All MD simulations were performed using NAMD2.12 [41] and CHARMM36 [42-44] force field. Following energy minimization, each system was equilibrated for $5 \mathrm{~ns}$ with position of heavy backbone atoms harmonically restrained $\left(k=2 \mathrm{kcal} / \mathrm{mol} / \AA^{2}\right)$. To facilitate the interaction with the lipid bilayer, we pulled the COM of the peptides towards the closest surface of the bilayer, defined by the phosphorus $(\mathrm{P})$ atoms of the corresponding leaflet. This was accomplished by applying a harmonic guiding potential $\left(k=1 \mathrm{kcal} / \mathrm{mol} / \AA^{2}\right)$, to the COMs of the first (residues 1-14, SEG1) and second (residues 15-26, SEG2) segment of the peptide, thus keeping it in a parallel conformation [91]. The center of the guiding potential was moved uniformly $(v=0.1 \mathrm{~nm} / \mathrm{ns})$ towards the plane of the bilayer. Once the peptide reached the surface of the bilayer, the guiding potential was kept fixed and its force 


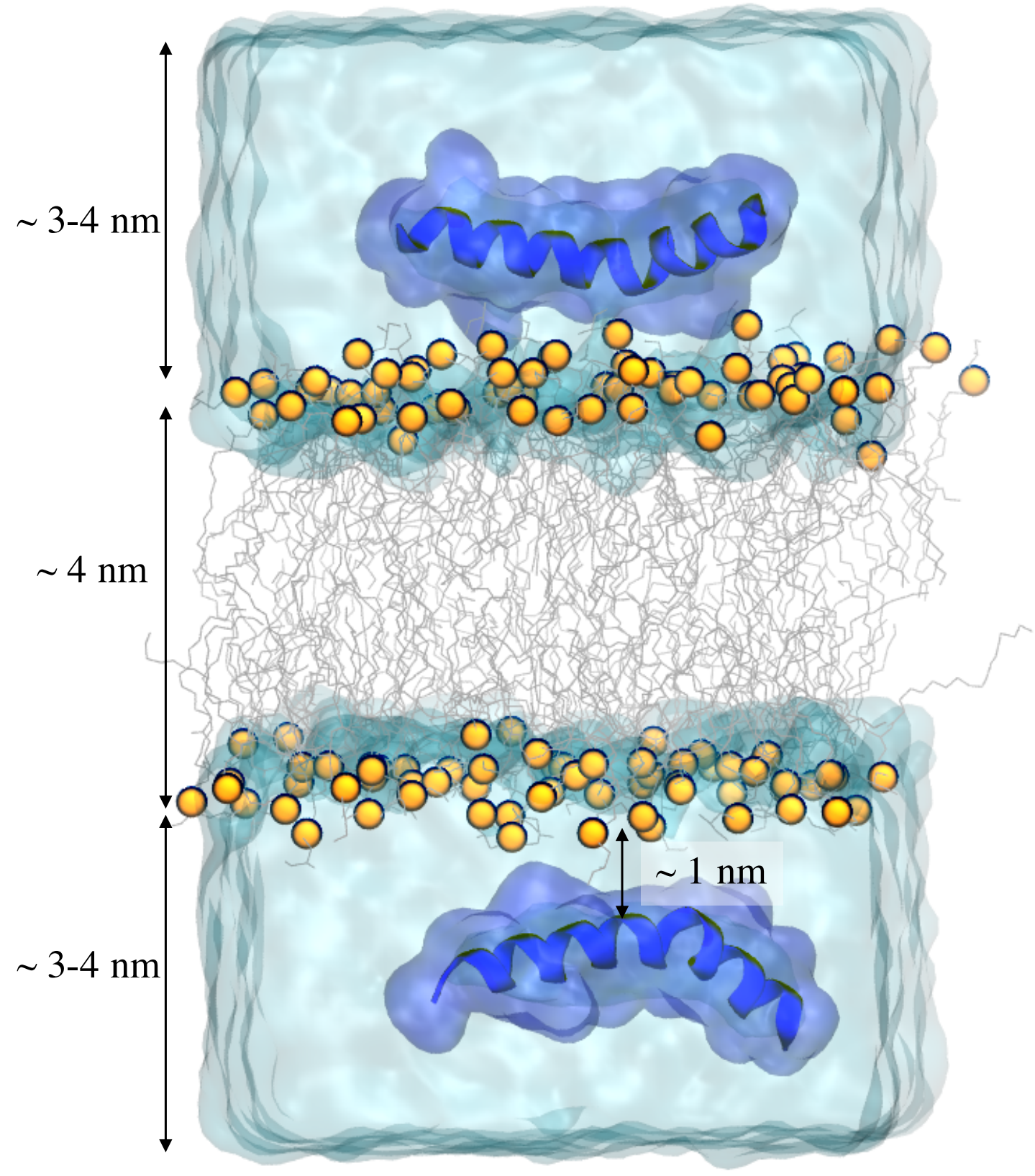

Figure 5.3: Initial configuration of the peptide-lipid bilayer system. Waters are shown as transparent surface (cyan), lipids as grey lines, and P-atoms as orange spheres. The folded peptides are shown in cartoon representation (blue helices); the transparent blue surfaces indicate the spatial extent of peptides' atoms. 
constant decreased gradually over a period of $10 \mathrm{~ns}$. The removal of the harmonic restraint was followed by free equilibration. All simulations (see Table 5.1) were performed in the NpT ensemble, using periodic boundary conditions (PBC) with flexible unit cell. In case of the MD4-PC system, a soft, reflective boundary $\left(k=1 \mathrm{kcal} / \mathrm{mol} / \AA^{2}\right)$ was placed at $z=2$ $\mathrm{nm}$ from the surface of the lipid bilayer on each side. This choice was based on the results obtained from a $600 \mathrm{~ns}$ long simulation we had originally ran, during which, neither one of MD4 peptide was interacting with the bilayer (Appendix D). In order to facilitate the interaction between the MD4 peptides and POPC lipid bilayer, we have applied harmonic boundaries, as described above. No restraints were applied in the other MD simulations.

The pressure was maintained constant $(\mathrm{p}=1 \mathrm{~atm})$ by employing the Nosé-Hoover Langevin piston method $[49,99,100]$, with100 fs period and decay time of $50 \mathrm{fs}$. By coupling the system to a Langevin thermostat ( $1 \mathrm{ps}^{-1}$ damping coefficient), the temperature was kept constant at $300 \mathrm{~K}$. Long-range electrostatic interactions were computed with the particle Mesh Ewald method [47]. The rRESPA multiple-timescale integration mehtod was used, with time steps of 1, 2, and 4 fs for bonded, short-range, and long-range interactions. Van der Waals interactions were cut off at $12 \AA$, with smooth switching function starting at $10 \AA$ A. A pair list distance of $13.5 \AA$ was used for smoothening non-bonded interactions.

\section{Umbrella sampling simulations}

Umbrella sampling (US) simulations [101] were used to compute the free energy profile and explore the dissociation of parallel peptide from the lipid bilayer surface. The simulations were carried out with the collective variables module [102] in NAMD [41].

Harmonic umbrella potentials, with $k=5 \mathrm{kcal} / \mathrm{mol} / \AA^{2}$, were used. The reaction coor- 
Table 5.1: List of all MD simulations of MWT and MD4. Simulations in aqueous solution are denoted with "sol", whereas "PC" and "PG" indicate POPG and POPC bilayers, respectively. Suffix "us" refers to umbrella sampling simulations.

\begin{tabular}{llcccc}
\hline Simulation & Peptide & Copies & Lipid & Time (ns) & Windows \\
\hline \hline MWT-sol & MWT & 1 & & 500 & \\
MD4-sol & MD4 & 1 & & 500 & \\
\hline MWT-PC & MWT & 2 & POPC & 400 & \\
MD4-PC & MD4 & 2 & POPC & 400 & \\
\hline MWT-PG & MWT & 2 & POPG & 400 & \\
MD4-PG & MD4 & 2 & POPG & 400 & \\
\hline MWT-PCus & MWT & 1 & POPC & 250 & 25 \\
MD4-PCus & MD4 & 1 & POPC & 250 & 25 \\
\hline MWT-PGus & MWT & 1 & POPG & 250 & 25 \\
MD4-PGus & MD4 & 1 & POPG & 250 & 25 \\
\hline
\end{tabular}

dinate (RC), $\Delta z \equiv z-z_{P}$, was defined as the separation between the COMs of SEG1 and $\operatorname{SEG} 2\left(z_{1}=z_{2} \equiv z\right)$ and the surface of the lipid bilayer $\left(z_{P}\right)$. Initially, the COM of the peptide was in the plane of the membrane (i.e., $z_{1_{0}}=z_{2_{0}}=z_{P}$ ). We used 25 sampling windows, evenly spaced by $1 \AA$ along the RC, which spanned the interval from 0 to $25 \AA$. Each window was sampled for $10 \mathrm{~ns}$ out of which the first $2 \mathrm{~ns}$ of the MD trajectories were discarded. Significant overlap between position histograms of adjacent windows showed that the RC was, indeed, well sampled. Finally, the PMFs were determined by applying Grossfield's implementation of the weighted histogram analysis method (WHAM) [103].

The MD simulations were carried out on several GPU accelerated workstations, each equipped with 36 Intel Xeon CPU cores, with a performance of around $32 \mathrm{~ns} /$ day. 


\subsubsection{Analysis methods}

In addition to free energy calculations, relative penetration depth and helical content were obtained. The degree of penetration was defined through the separation between the COM of SEG1/SEG2 from the COM of lipid bilayer. All analyses were done with VMD [37], MDAnalysis [50,51] and MDTraj [52]. Besides DSSP algorithm [53], a standard method for assigning secondary structure to the amino acids of a protein $[56,58,59]$, previous publications also calculated number of hydrogen bonds (usually formed between $\mathrm{CO}_{n}$ and $\mathrm{NH}_{n+4}$ backbone groups) and root mean square deviation (RMSD) [57]. We also applied the following methods: (i) STRIDE [104] (implemented through VMD [37]) and (ii) "custom", i.e. the residues whose backbone dihedral angles deviate $\pm 15^{\circ}$ from the ideal $\alpha$-helical values $\left(-57^{\circ},-47^{\circ}\right)$ were considered to contribute to total helical content [85]. All the methods gave very similar results, some of which will be reported in the following section. However, we found DSSP to be the most consistent (i.e., no sudden jumps over short periods of time), which is why it was used for further analysis. 


\subsection{Results and Discussion}

\subsubsection{MWT and MD4 in solution}

MWT is typically unstructured in solution. After 500 ns of simulations, more than $50 \%$ of MWT and $10 \%$ of MD4 was to found to be a random coil (Fig. 5.4a). Helical content of SEG1 dissolved more rapidly than it was the case for SEG2 (Fig. 5.4a-b). Nonetheless, in case of MD4, both segments appeared to be unstructured after 350 ns (Fig. 5.4c). Notable difference observed between MD4 and MWT can only be due to the presence of D-enatiomers (i.e., D-Val ${ }^{5,8}, \mathrm{Ile}^{17}$ and $\mathrm{Lys}^{21}$ ) in MD4.

Fig. 5.5 illustrates how much the helical content along peptide's sequence changed, on average, during the first and last $100 \mathrm{~ns}$ of total simulation time. Note that according to our results (Fig. 5.5), it would be more natural to define SEG1 as residues 1-12, and SEG2 as residues 13-26. However, we will continue to refer to SEG1 and SEG2 as they were originally defined in 5.2.2 [91].

Fig. 5.6 shows good overall agreement between results obtained with DSSP algorithm [53] and "custom" (described in 5.2.3 section) method (average difference was 6.8\%). Additionally, we calculated RMSD of SEG1 and SEG2 for both peptides. Increase of RMSD indicates less compact structure and as such is directly correlated with helical content (Fig. 5.7).

\subsubsection{MD simulations of MWT and MD4 partitioning into POPC lipid bilayer}

$400 \mathrm{~ns}$ of free simulation time was performed for each of MWT-PC and MD4-PC systems. 2/128 protein-to-lipid ratios were used for better statistics. Interestingly, in the case of 


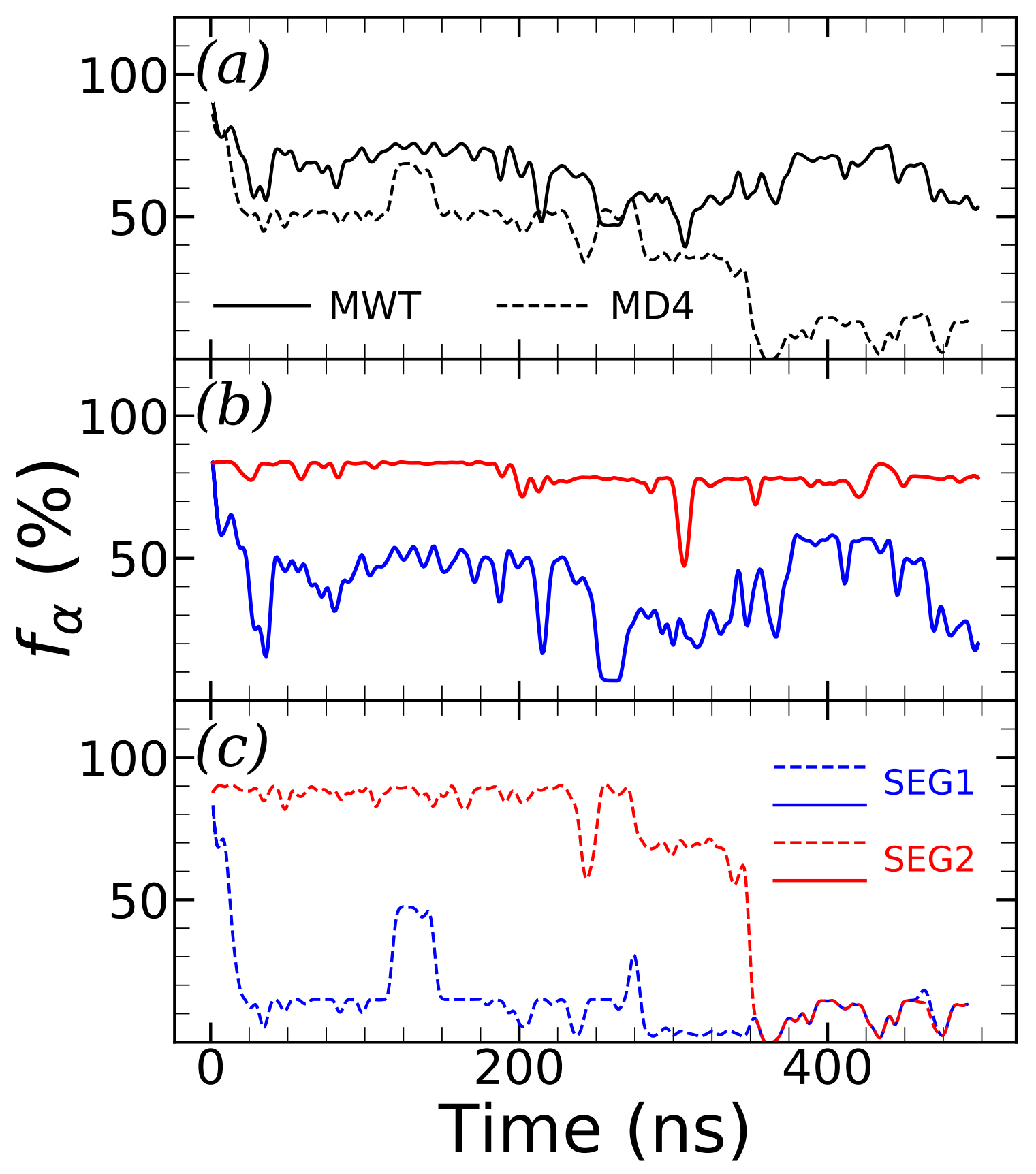

Figure 5.4: (a) Helical content of MWT (black solid line) and MD4 (black dashed lines). Time evolution of $\alpha$-helical helical content of SEG1 (blue) and SEG2 (red) of MWT (b) and MD4 (c). 


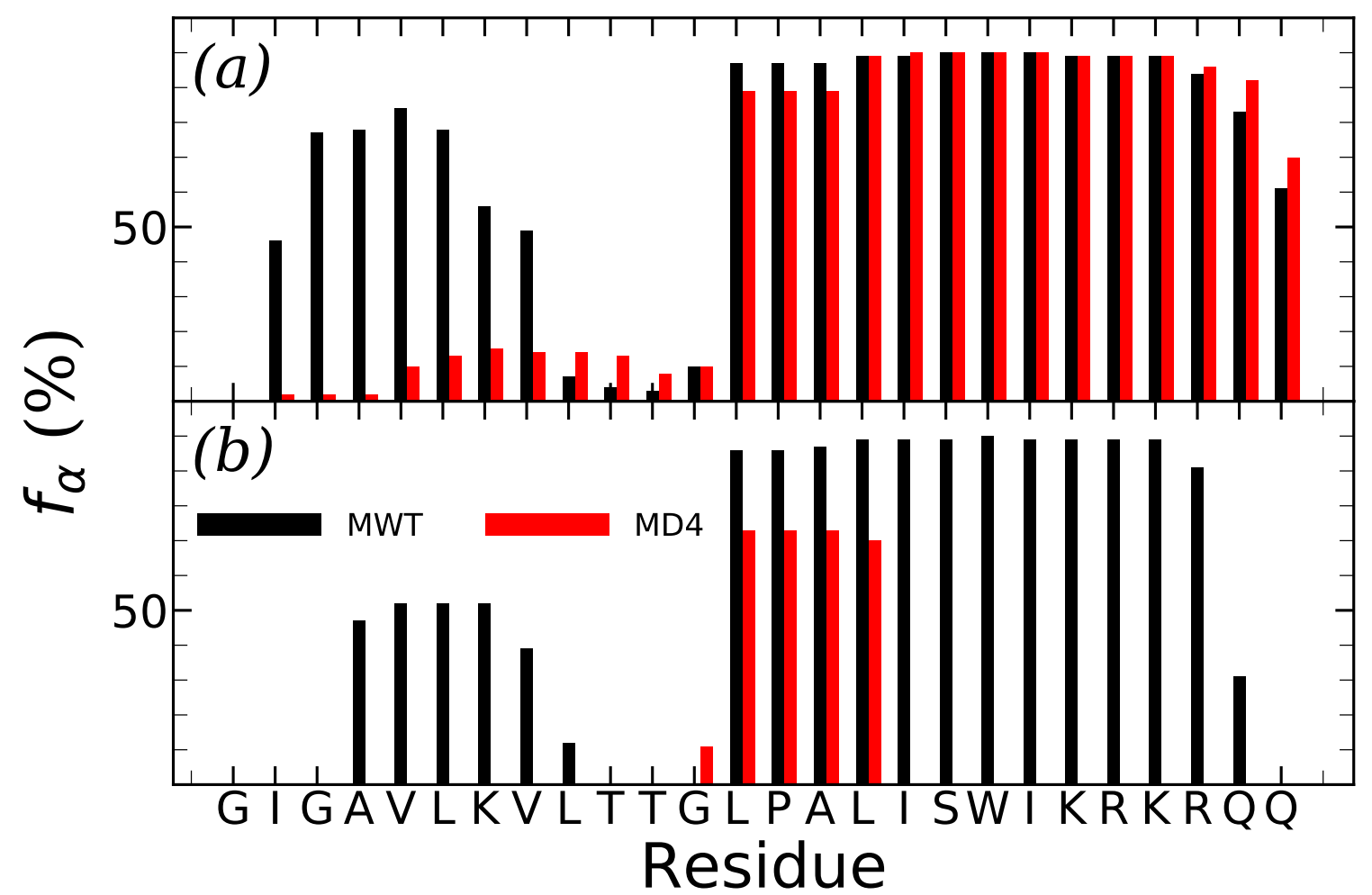

Figure 5.5: Helical content along the sequence of MWT (black) and MD4 (red). (a) Time average over first $100 \mathrm{~ns}$ and $(b)$ last $100 \mathrm{~ns}$, of total simulation time $(0.5 \mu s)$. 


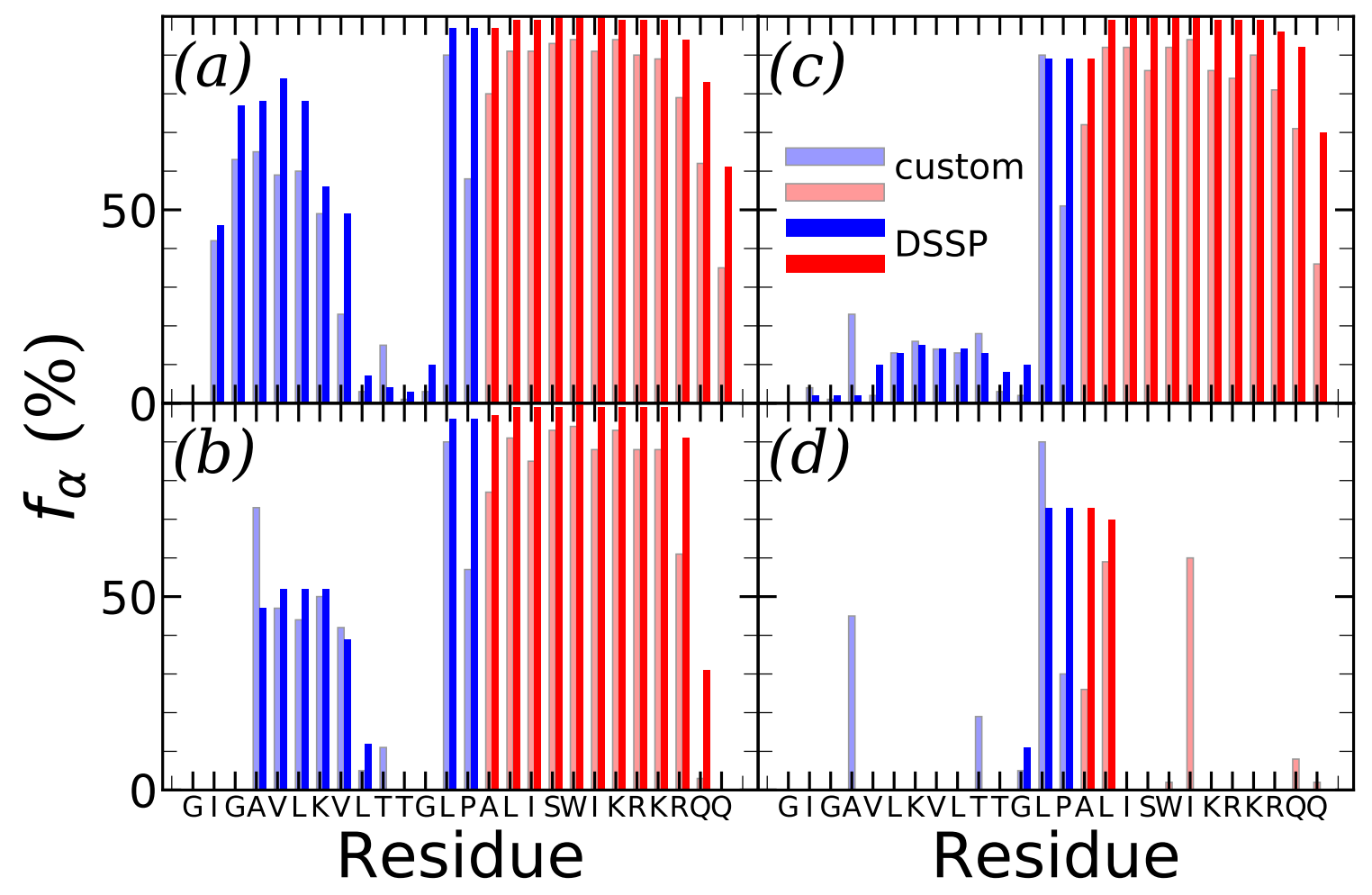

Figure 5.6: Helical content along the peptide sequence, compared between DSSP [53] (bright colors) and "custom" (faded colors) methods. (a) and (b) refer to MWT in solution, during the first and last $100 \mathrm{~ns}$, respectively, whereas $(c)$ and $(d)$ refer to MD4. SEG1 is colored in blue and SEG2 in red. 


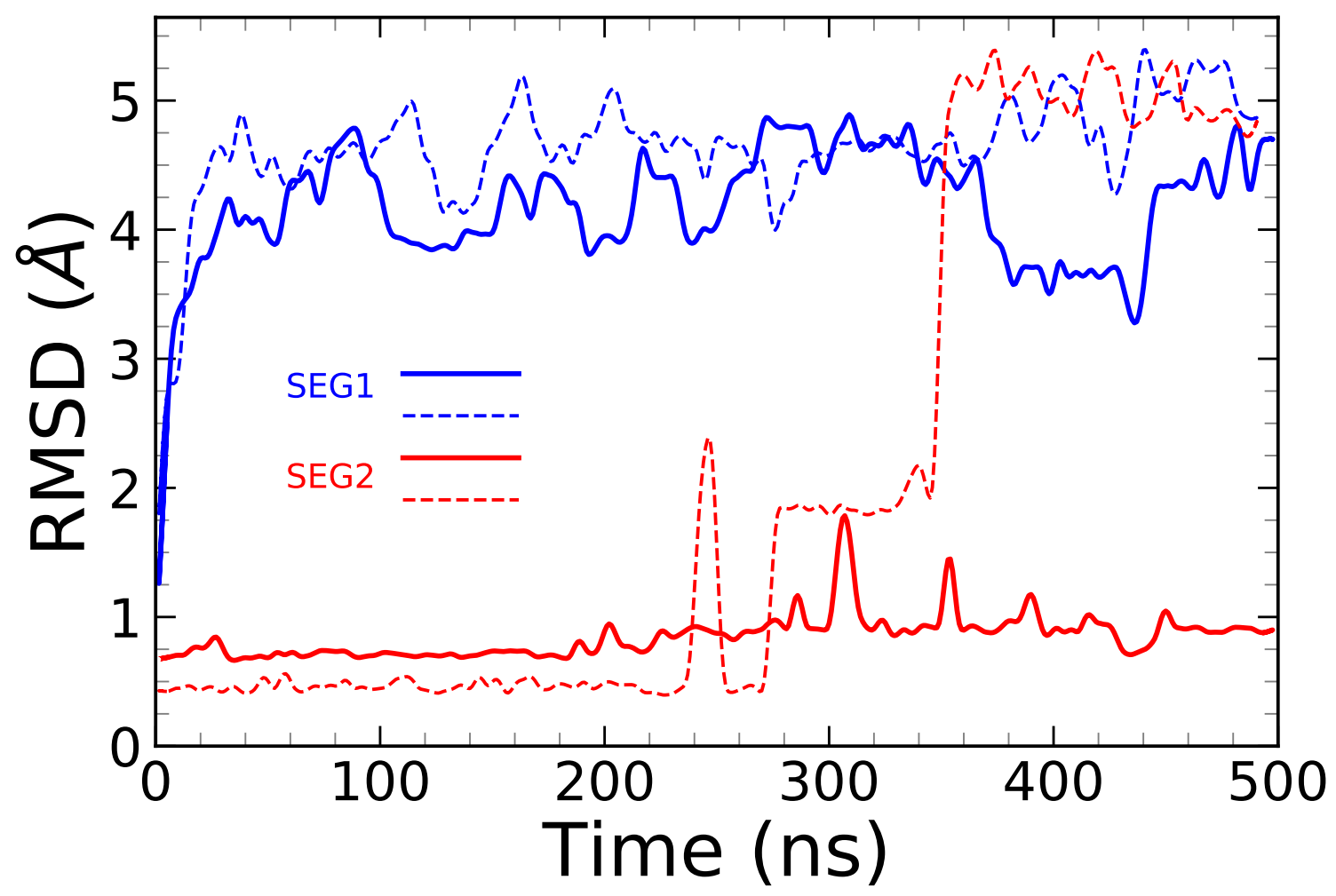

Figure 5.7: Time evolution of RMSD of MWT (solid lines) and MD4 (dashed lines) peptides from their initial structures. Blue corresponds to SEG1 and red to SEG2 of each peptide. 
MWT only one peptide copy was found in the bound state (Fig. 5.8a-b). Furthermore, in case of MD4 spontaneous insertion never took place (Fig. 5.8c-d), which is an expected outcome according to the experimental results [3].

To facilitate the binding of MD4 to POPC lipid bilayer, a harmonic reflective wall $(k=1$ $\mathrm{kcal} / \mathrm{mol} / \AA^{2}$ ) was placed at $2 \mathrm{~nm}$ from lipid-phosphate centers. Thus, the COM of each copy of MD4 thus remained in close proximity of the bilayer throughout the simulation $\left(z_{1}=z_{2}=4.0 \pm 0.6 \mathrm{~nm}\right.$ from the bilayer COM). However, to consider insertion of a peptide into a bilayer, $\mathrm{COM}$ of a peptide needs to be found below the plane formed by phosphorus atoms (i.e., $z_{P} \approx 2 \mathrm{~nm}$ ) over extended period of time, which never took place for any of the peptides in MD4-PC system. Helical content of top (bottom) MD4 decreased to 53\% (59\%). Note that bottom MD4 copy had $<10 \%$ of helical content during last 40 ns of the free production run (Fig. 5.9c-d).

Conversely, in the case of MWT-PC, the bottom MWT copy positioned itself below the headgroup region after $100 \mathrm{~ns}$, where it stayed throughout the simulation. Hydrophobic SEG1 of bottom MWT had the mean z-coordinate $z_{1}=-12.7 \AA$, while hydrophilic SEG2 was anchored at $z_{2}=-19.3 \AA$ (i.e., $6.9 \AA$ and $0.3 \AA$, respectively, below the lipidphosphate centers). The top copy of MWT never partitioned the same bilayer, which may be due to the electrostatic repulsion, how the size of our system (i.e., membrane patch) was relatively small. Consequentially, bottom MWT had high helical content (over 80\%), while the helical content of the top MWT decreased as the separation of the peptide from the COM of lipid bilayer increased, with an average of $70 \%$ over the entire simulation time (Fig. 5.9a-b).

Similar results were obtained after $600 \mathrm{~ns}$ of free simulation time which involved $+5 \mathrm{e}$ charged MWT and MD4 peptides (i.e., with standard, deprotonated C-terminus) and POPC 


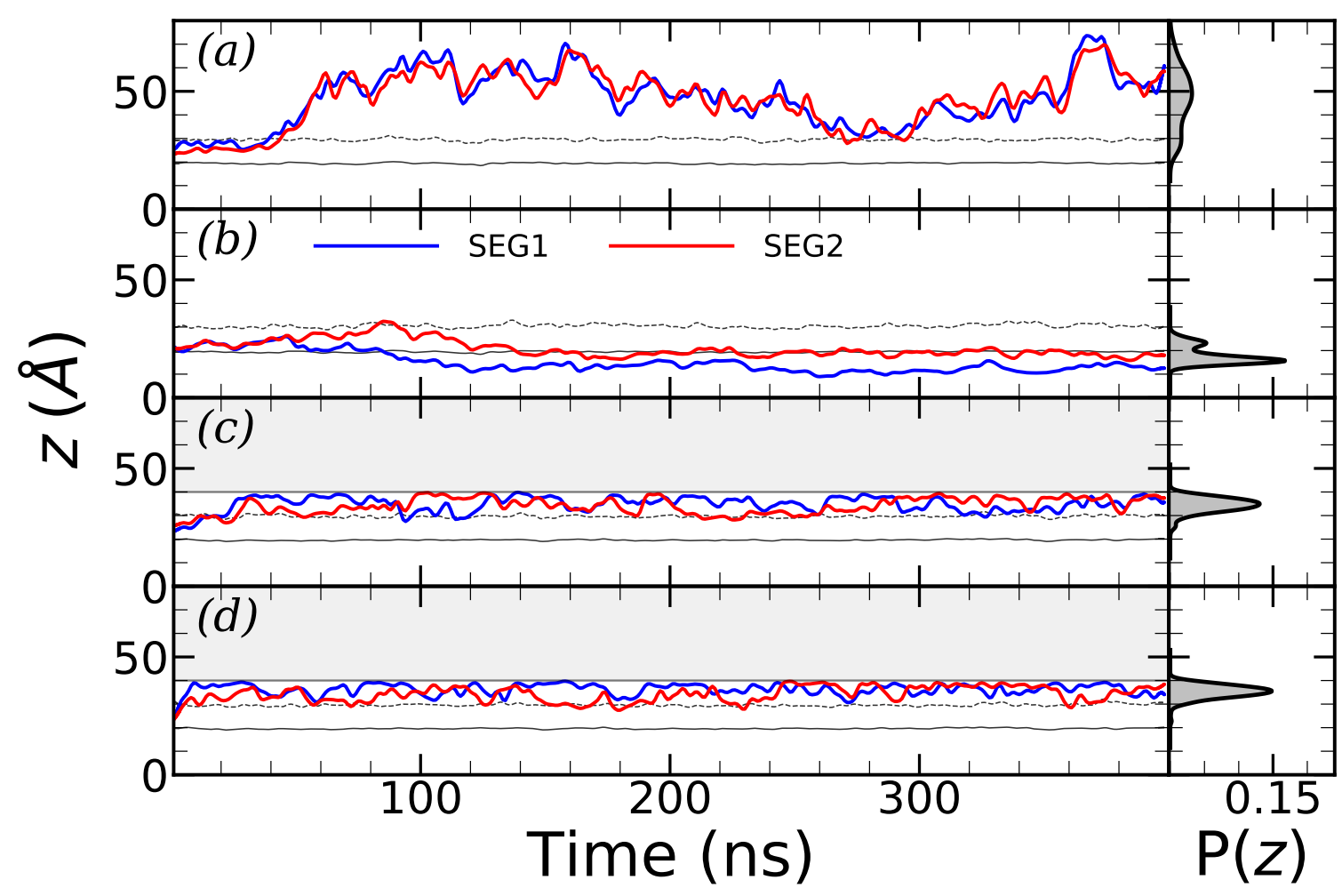

Figure 5.8: Time evolution (panels on the left) and cumulative histograms (panels on the right) of separation $(z)$ between SEG1 (blue) and SEG2 (red) of MWT $((a)-(b))$ and MD4 $((c)-(d))$ and COM of POPC lipid bilayer. Panels on the left also show the time evolution of POPC lipid-phosphate centers (black solid line) and maximum $z$-coordinate for all lipid atoms (black dashed line), located at $19.5 \AA$ above (below) the COM of the bilayer.

lipid bilayers. Additionally, we simulated a single MWT copy and POPC lipid bilayer. Single (and only) copy of MWT spontaneously inserted itself into the bilayer on a time scale $<50$ ns (more in Appendix D).

\subsubsection{MD simulation of MWT and MD4 partitioning into POPG lipid bilayer}

MWT-PG and MD4-PG systems were simulated for total of $400 \mathrm{~ns}$, during which, distances of COMs of SEG1 and SEG2 relative to the COM of POPG lipid bilayer were analyzed so 


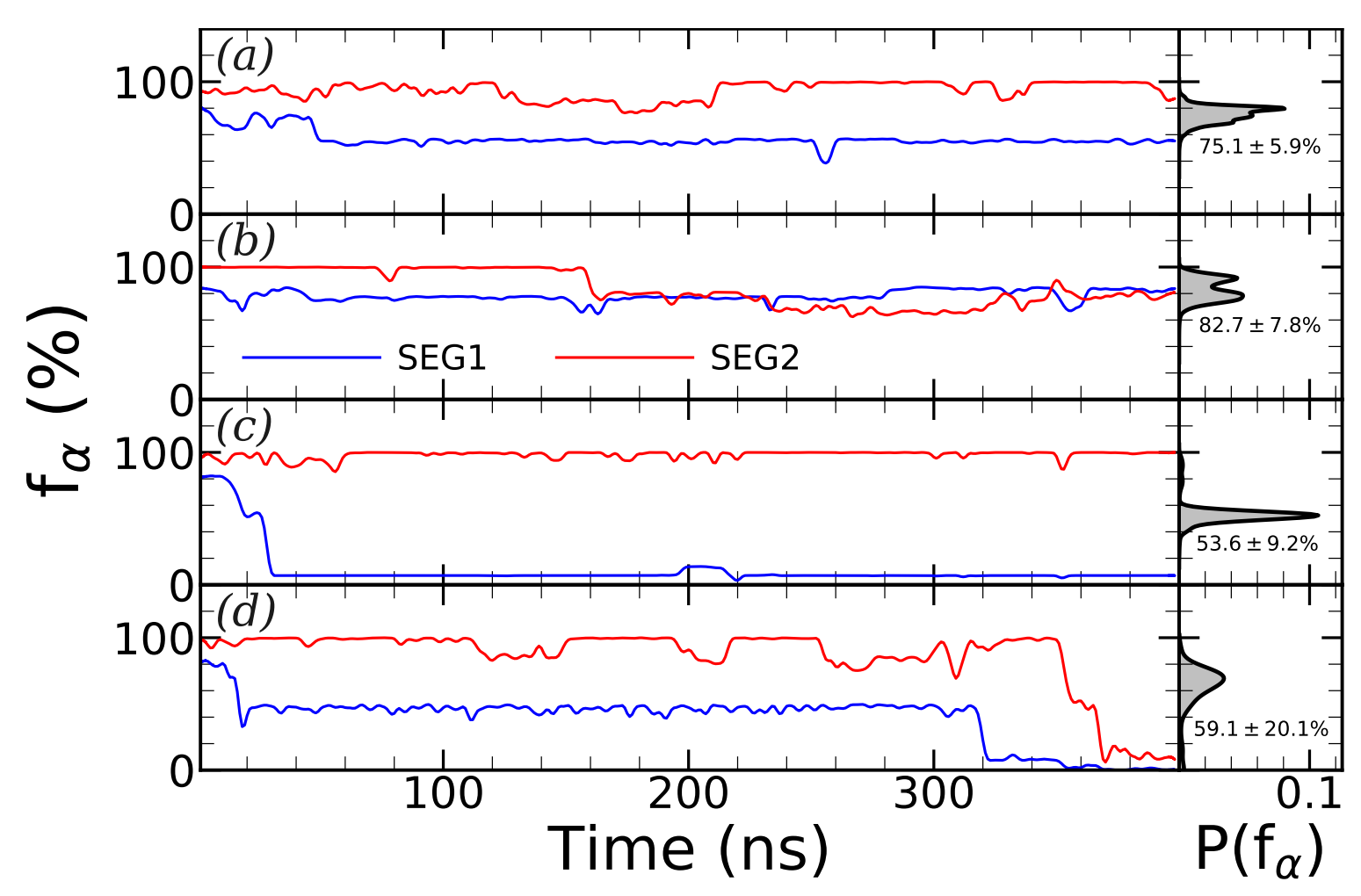

Figure 5.9: Time evolution of helical content of MWT and MD4 peptide in MWT-PC and MD4-PC systems, calculated according to the DSSP algorithm [53]. (a) and (b) refer to top and bottom copy of MWT. $(c)$ - (d) refer to the 2 copies of MD4. Panels on the left show how helical content of SEG1 (blue) and SEG2 (red) was changing over total simulation time. On the right are cumulative histograms of helical content of the entire peptides. 
we could establish the degree of interaction between the peptides and the bilayer. Corresponding helical content was also calculated.

After $\sim 100 \mathrm{~ns}$, top MWT was spontaneously inserted into the bilayer. Particularly, COM of hydrophobic SEG1 $\left(z_{1}=12.96 \AA\right)$ penetrated deeper in the core of POPG bilayer, than it was the case for COM of charged SEG2 $\left(z_{2}=18.87 \AA\right)$, which remained anchored in the bilayer headgroup region. Bottom MWT stayed in the head-group region with COM of SEG1 and SEG2 at $z_{1}=-25.83 \AA$ and $z_{2}=-23.48 \AA$, respectively, below the COM of POPG bilayer (Fig. 5.10a-b). Helical content of both MWT peptides remained high (75.53\% and $77.46 \%$, for top and bottom copy, respectively). These results are comparable to the ones obtained in POPC (Fig. 5.11a-b).

In contrast to MD4-PC, both copies of MD4 stayed relatively close to POPG lipidphosphate centers, with $z_{1}=18.99(-15.03) \AA$ and $z_{2}=17.27(-21.43) \AA$ from the center of POPG bilayer for top (bottom) peptide (Fig. 5.10c-d). Nevertheless, helical content decreased to $43.07 \%$ (46.81\%) for the top (bottom) MD4 (Fig. 5.11c-d). Similar to the results in solution and POPC bilayer (i.e., top MD4), hydrophilic SEG2 had higher helical content than the hydrophilic SEG1.

\subsubsection{Potential of Mean Force (PMF)}

We preformed US simulations for a total of $1.04 \mu$ s for all the systems (Table 5.1), in order to quantify the interaction between peptides (i.e., MWT and MD4) and lipid bilayers (i.e., PC and PG). Each PMF was calculated as a function of separation between COM of SEG1 and SEG2 of each peptide and lipid-phosphate centers (Fig. 5.12). Value of $U(z)$ in the solution was taken as zero.

Free energy profiles obtained for MWT-PC and MD4-PC systems were qualitatively 


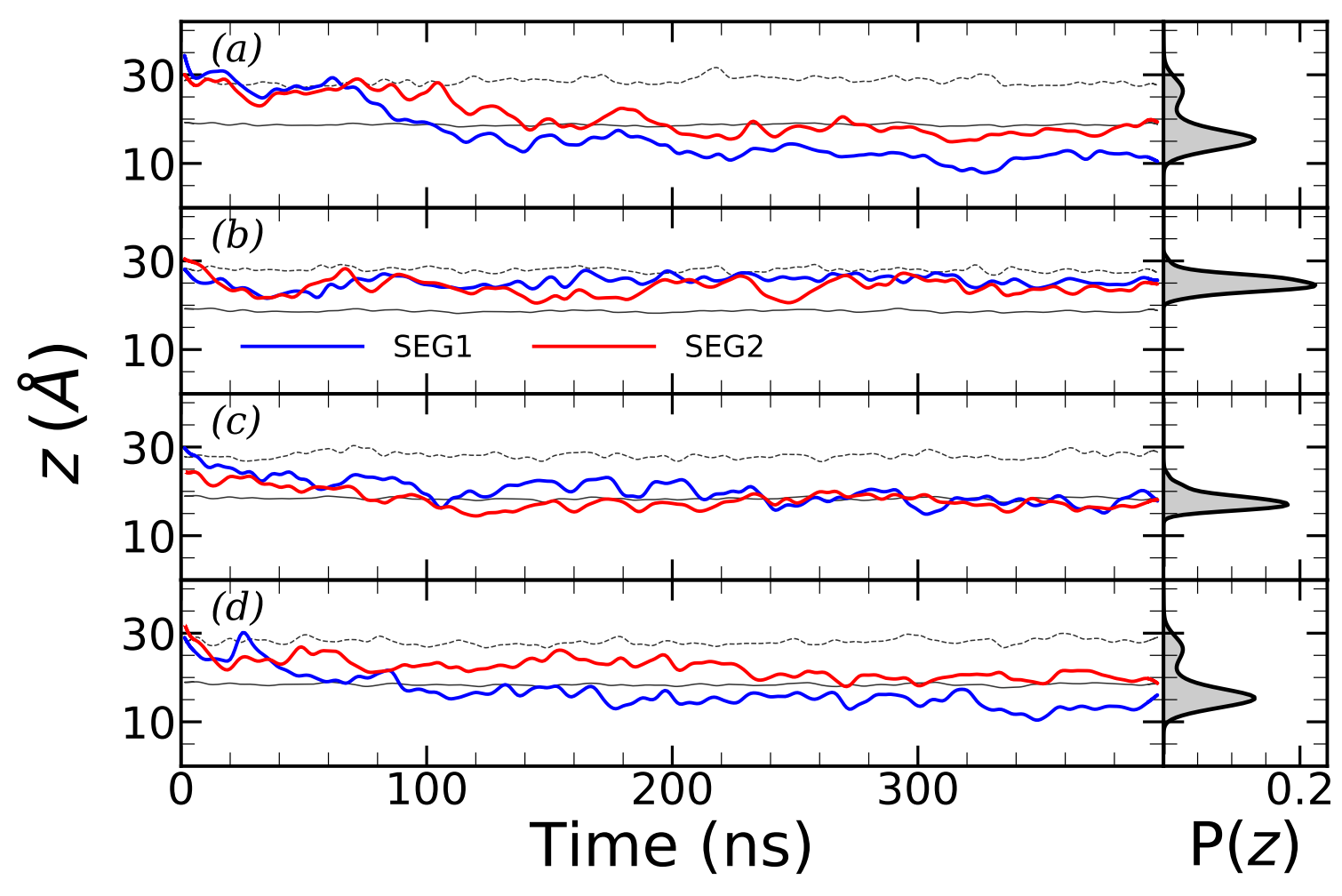

Figure 5.10: Time evolution (panels on the left) and cumulative histograms (panels on the right) of separation ( $z$ ) between SEG1 (blue) and SEG2 (red) of MWT ((a) - (b)) and MD4 $((c)-(d))$ and COM of POPG lipid bilayer. The time evolution of POPG lipid-phosphate centers (black solid line) show them being located at $18.7 \pm 0.3 \AA$ above (below) bilayer's COM, while maximum $z$-coordinate of all lipid atoms (black dashed line) was located $\sim 1$ $\mathrm{nm}$ above (below) the latter. 


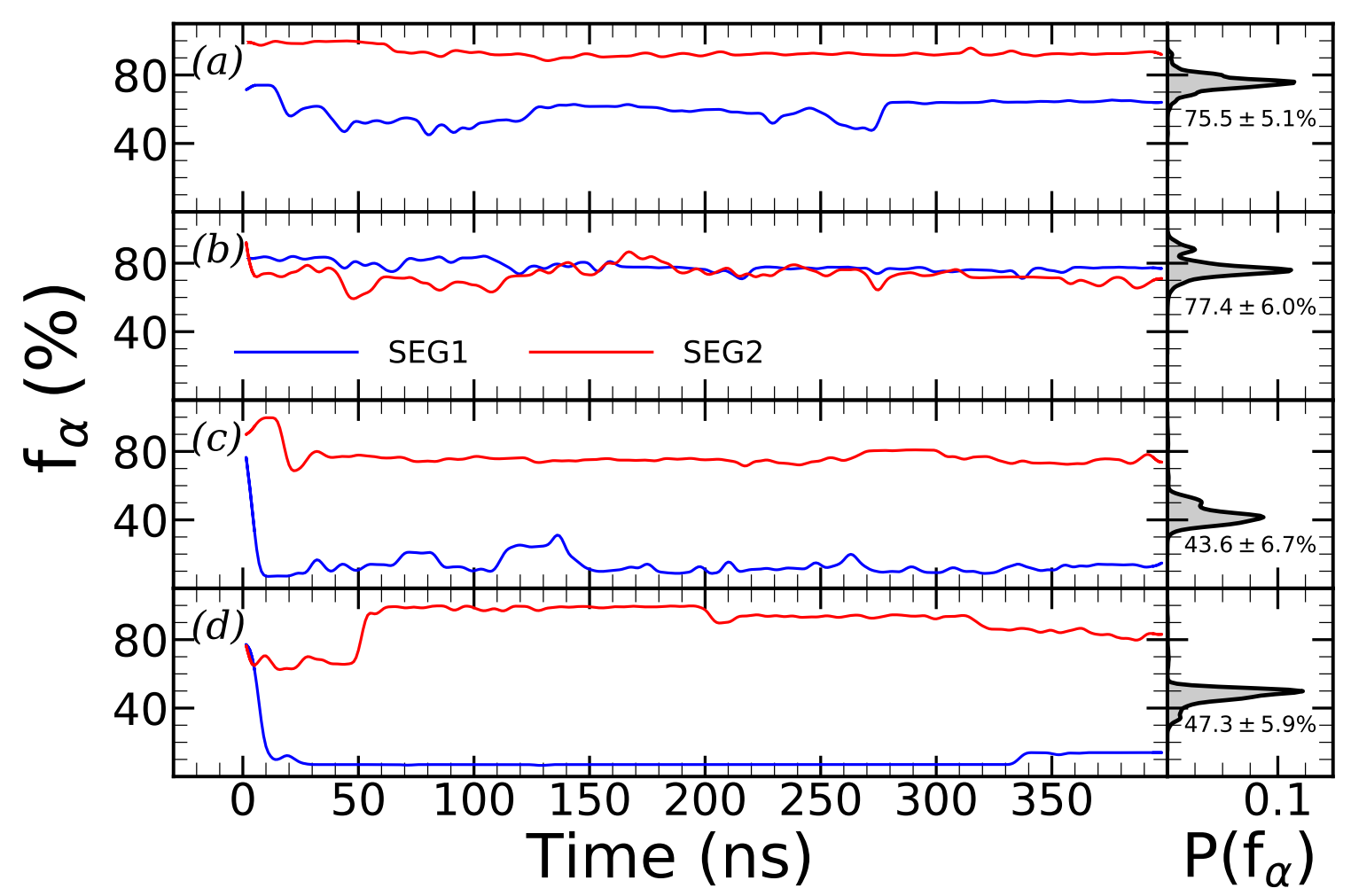

Figure 5.11: Helical content of peptides in MWT-PG $((a)-(b))$ and MD4-PG $((c)-(d))$, calculated for SEG1 (blue) and SEG2 (red) with DSSP algorithm [53]. On the right are cumulative histograms of helical content for the entire peptides. 
different. Namely, in case of MD4, there was a free energy barrier of $\approx 5 \mathrm{kcal} / \mathrm{mol}$, located in the headgroup region of POPC lipid bilayer. This was consistent with the experimental findings [3], as well with our previously obtained results from the free energy simulations. By contrast, dissociation energy of $\approx-15 \mathrm{kcal} / \mathrm{mol}$ indicated strong affinity of MWT to stay in the bound state. These results also seem to be in agreement of relative penetration depths (Fig. 5.8), as one might expect.

On the other hand, both MWT and MD4 peptides showed strong affinity towards the POPG bilayer. Dissociation energies of MWT-PG and MD4-PG systems were approximately $-21 \mathrm{kcal} / \mathrm{mol}$ and $-15 \mathrm{kcal} / \mathrm{mol}$, respectively. Stronger interaction in case of MWTPG can be attributed to 35\% higher helical content than in MD4-PG system. Furthermore, there is a direct relationship between $\alpha$-helical content and number of hydrogen bonds, that promote the secondary structure. Thus, we calculated the number of inter-peptide hydrogen bonds from the obtained US trajectories. Total number of hydrogen bonds was then averaged over $10 \mathrm{~ns}$, during which each window was sampled (Fig. 5.13). Lowest energy associated with a hydrogen bond is approximately $1 \mathrm{kcal} / \mathrm{mol}$ [105]. Due to criteria that was used for estimation of number of hydrogen bonds, we are on the lower end of the scale. Finally, the difference in the dissociation energies of $6 \mathrm{kcal} / \mathrm{mol}$, extracted from PMFs, directly relates to the difference in the number of hydrogen bonds, which was 6 .

In addition, figs. 5.14 and 5.15 compare results obtained from US trajectories with the free simulations results. The difference in the number of hydrogen bonds was $\sim 8$ (6) in case of PC (PG) systems. 


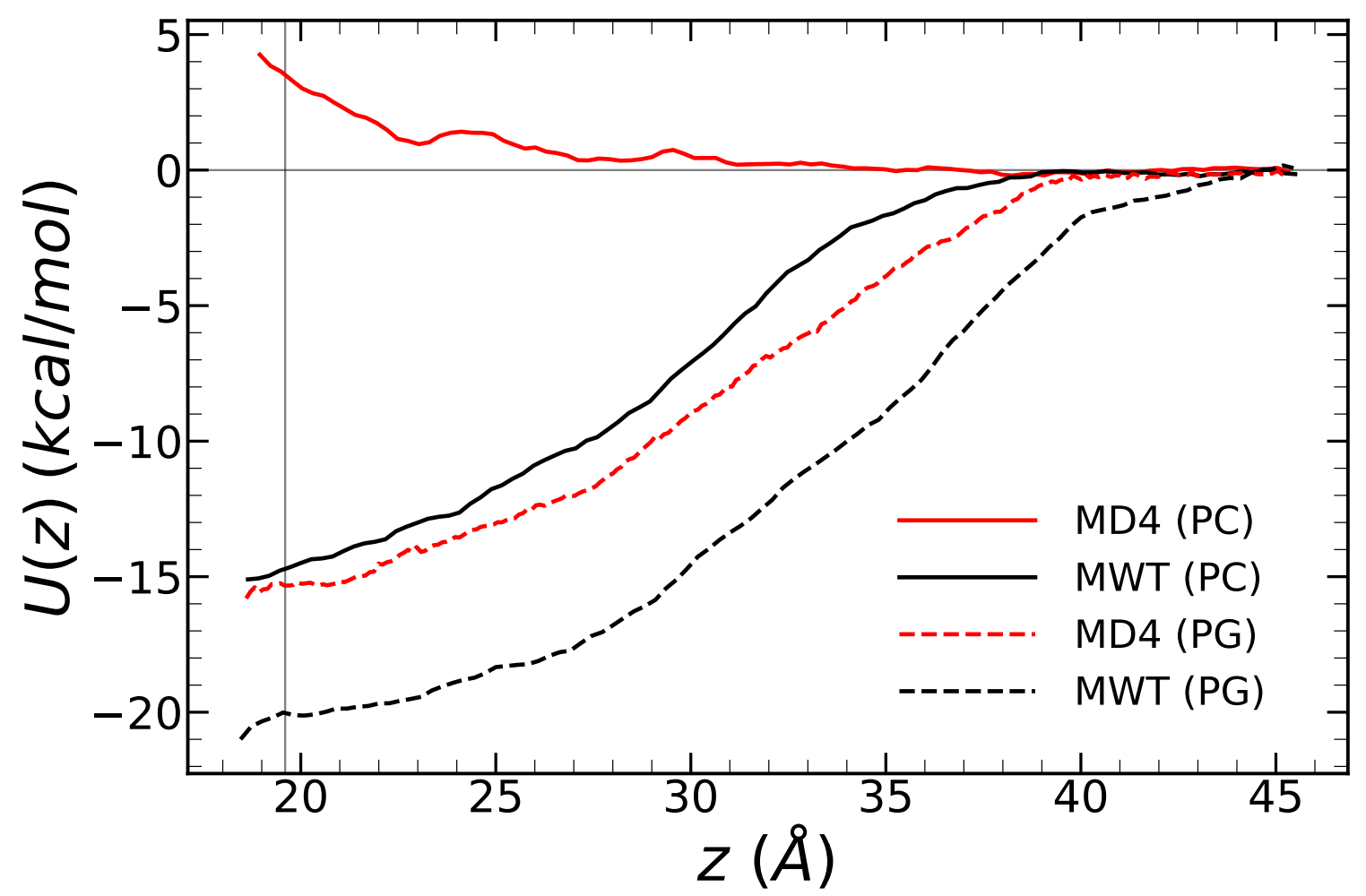

Figure 5.12: Potentials of mean force for MWT (black) and MD4 (red) in POPC (full line) and POPG (dashed line) lipid bilayers, as a function of separation between COM of SEG1 (SEG2) and phosphate centers of POPC and POPG bilayers. The separation $(z)$ uniformly increased from $20 \AA$ to $45 \AA$. Vertical (grey) line at $20 \AA$ indicates the position of lipidphosphate centers. 


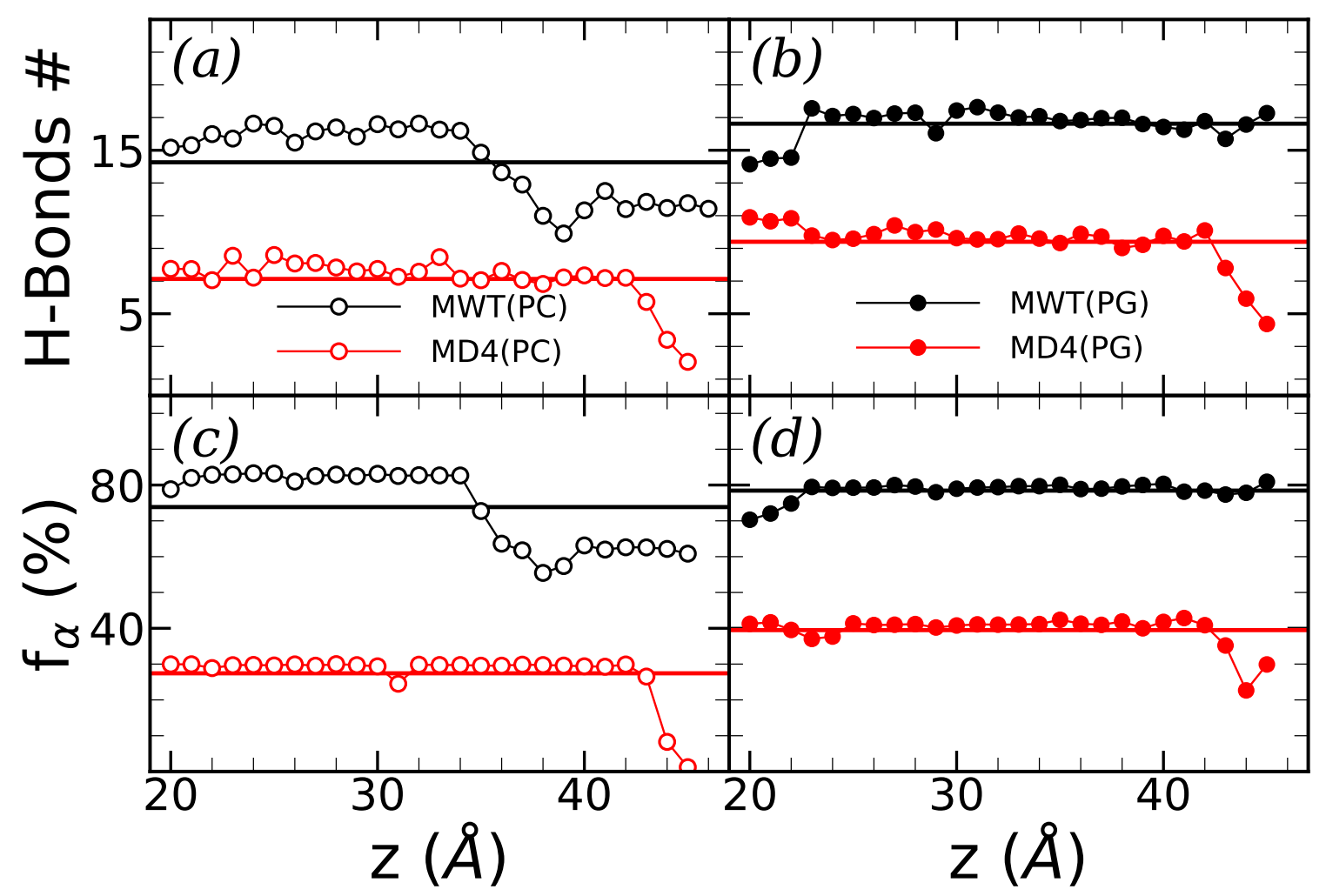

Figure 5.13: Number of hydrogen bonds and helical content of MWT (black) and MD4 (red) peptides in POPC (hollow circles) and POPG (filled circles) lipid bilayer during US simulations. (a) Number of hydrogen bonds and (c) helical content of MWT and MD4 in POPC. Similarly, $(b)$ the number of hydrogen bonds and $(d)$ helical content of MWT and MD4 in POPG. 


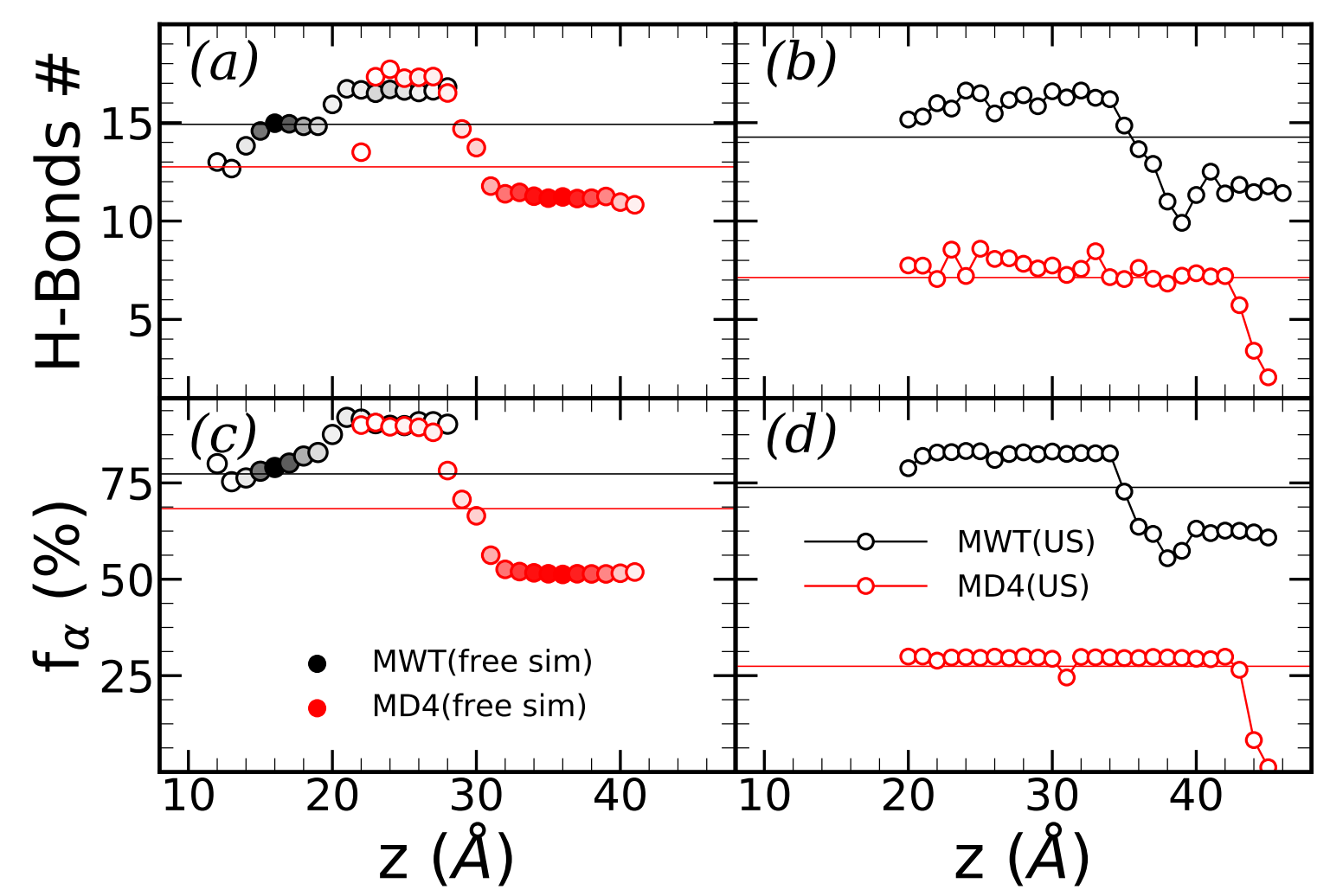

Figure 5.14: Number of hydrogen bonds and helical content of MWT (black) and MD4 (red) in POPC lipid bilayer and as a function of distance from COM of the bilayer, compared between free production runs and US simulations. (a) Number of hydrogen bonds for MWT (circles colored with shades of black) and MD4 (circles colored with shades of red). Positions that were visited more frequently during the free simulation correspond to darker shades of color. (b) Number of hydrogen bonds during the US simulation of MWT (hollow black circles) and MD4 (hollow red circles). (c) Helical content of MWT and MD4 peptides during free simulation and, similarly, $(d)$ helical content of MWT and MD4 peptides during US simulations. 


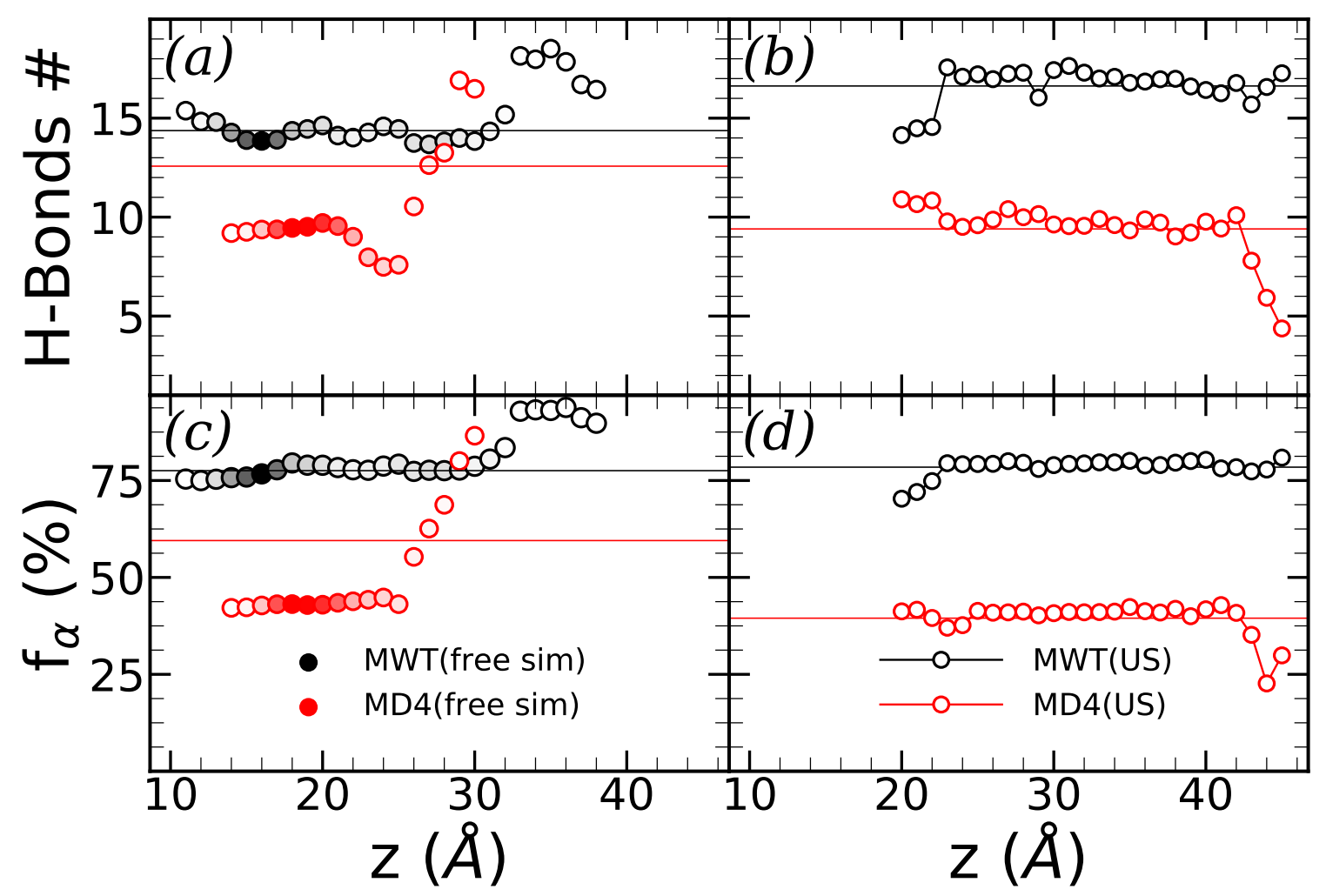

Figure 5.15: Number of hydrogen bonds and helical content of MWT (black) and MD4 (red) in POPG lipid bilayer and as a function of distance from COM of the bilayer, compared between free production runs and US simulations. (a) Number of hydrogen bonds for MWT (circles colored with shades of black) and MD4 (circles colored with shades of red). Positions that were visited more frequently during the free simulation correspond to darker shades of color. (b) Number of hydrogen bonds during the US simulation of MWT (hollow black circles) and MD4 (hollow red circles). (c) Helical content of MWT and MD4 peptides during free simulation and, similarly, $(d)$ helical content of MWT and MD4 peptides during US simulations. 


\subsection{Conclusions}

In order to assess the importance of the secondary structure in peptide-lipid membrane interactions, we needed to directly compare the interaction between peptides of same primary but different secondary structures and two different types of lipid bilayers. Since MD4 is melittin's diasteromer, the two peptides (i.e., MD4 and MWT), naturally, have same primary structure. After completing all of our MD production runs, a significant difference in helical content between the two peptides was observed. MWT kept majority of its helical content ( $\sim 80 \%)$, unless in aqueous solution. By contrast, MD4 was mostly unstructured, which was independent on the proximity of a bilayer. These results indicated that MWT and MD4, indeed, were an ideal choice for our study.

Our free MD simulations supported the results of previous studies, according to which MWT binds to and assumes parallel orientation in both zwitterionic POPC and anionic POPG bilayer. By contrast, MD4 only binds to the latter. To the best of our knowledge, this is the first MD study of MD4.

The affinity of the peptides for different bilayers was further quantified by calculating

the corresponding PMFs. In each of the US simulations, we started with peptides in a parallel bound conformation. MWT-PC and MD4-PC systems had qualitatively different PMFs due to absence of a free energy well in case of the latter. This was in agreement with results obtained from the free MD simulations, during which, MD4 was never found in the bound state.

Difference of $\sim 5 \mathrm{kcal} / \mathrm{mol}$ in dissociation energies was obtained between MWT-PG and MD4-PG systems, even though both MD4 and MWT interacted with POPG bilayer during the free MD simulations. Furthermore, after the number of hydrogen bonds was calculated from same US simulations, we found that MWT had 6 inter-peptide hydrogen bonds more 
than MD4 peptide. Finally, if energy of $1 \mathrm{kcal} / \mathrm{mol}$ is associated with a single hydrogen bond, then hydrogen bonding energy directly correlates to the difference in dissociation energies extracted from the PMFs. 


\section{Chapter 6}

\section{Summary and concluding remarks}

This thesis presents three interrelated theoretical and computational studies involving peptidelipid membrane interactions, a topic of great conceptual and practical importance in molecular biology and biophysics. A brief summary of the obtained results follows.

First, we have formulated a general theoretical approach that provides a quantitative description of the detachment force distribution of a peptide from a lipid membrane [1]. The proposed model assumes that detachment occurs, with certain probability $\left(w_{n}\right)$, along a few dominant diffusive pathways, characterized by three parameters $\left(\Delta U_{0 n}, \Delta x_{0 n}\right.$ and $\left.k_{0 n}\right)$. We identified these pathways as ruptures involving single or double residues, most likely located at the end of a peptide. The values of the energetic $\left(\Delta U_{0 n}\right)$ and geometric $\left(\Delta x_{0 n}\right)$ parameters, which are residue and lipid species specific, can be obtained from existing MD studies [26,27]. The kinetic parameters $\left(k_{0 n}\right)$ and pathway weights $\left(w_{n}\right)$ can then be determined by fitting the theoretical prediction to the experimental $P(F)$. The multiple detachment pathways model was validated in the forced dissociation of the peptide SecA2-11 from a zwitterionic POPC and a polar E. coli lipid membranes. Even though the measured 
$P(F)$ histograms differed considerably for different lipids, AFM tips (prepared under identical conditions) and retraction speeds of the AFM cantilever, the experimental data was interpreted in a consistent manner by assuming $N=4$ pathways (i.e., two single- and two double-residue ruptures). Once the pathway specific parameters $\Delta U_{0 n}, \Delta x_{0 n}$ and $k_{0 n}$ were determined for a given peptide-lipid bilayer system (apart from minor adjustment of the intrinsic dissociation rates, $k_{0 n}$ ), the fitting of the experimental detachment force histograms, $P(F)$ s, simply required the recalculation of the weight factors, $w_{n}$. Retraction speed and non-monotonic force dependence of the corresponding rupture rate, $k(F)$, was also accurately reproduced. Finally, we found that the peptide-lipid membrane dissociation (off-) rate, $k(0)$, was independent (within margin of errors) of the retraction speed, in spite of the significant $v$-dependence of both $P(F)$ and $k(F)$.

Second, in order to gain insight into the conformational dynamics of SecA2-11 during its interaction with zwitterionic POPC and anionic POPG lipid bilayers, we have performed long time scale, all-atom MD simulations. We have found that SecA2-11 in POPG became more compact, intermittently forming a helical turn, while in POPC remained mostly a random coil. These findings are consistent with $\mathrm{CD}$ measurements, which indicate the presence of secondary structure elements in SecA2-11 when interacting with E. coli, but not with POPC. Furthermore, the mean penetration depths of the individual SecA2-11 residues, determined from MD simulations, were in good overall agreement with previously published power saturation electron paramagnetic resonance results for the full length SecA in E. coli membrane [36], suggesting that the extreme $\mathrm{N}$-terminus of SecA indeed acts as an independent structural entity through which the protein binds to the cell membrane. This result lends further support to our theoretical and computational approach for describing peptide-lipid membrane interactions. 
Finally, in third project, we performed a comparative MD study of the conformational dynamics and energetics of (wild type) melittin (MWT) and its diasteromer (MD4) interacting with a zwitterionic POPC and a charged (anionic) POPG lipid bilayer. We showed that MWT binds to both POPC and POPG, remaining mostly folded (with $\gtrsim 80 \%$ helical content). By contrast, we found that MD4 binds only to POPG and it is always disordered. These results are in agreement with previous experimental studies [3]. Furthermore, to the best of our knowledge, this is the first long time scale, all-atom MD simulations study of the MD4 peptide. In addition, the dissociation energies of MWT and MD4 (oriented parallel to the surface of the membrane) from POPC and POPG were extracted from the potentials of mean force (PMFs) determined from umbrella sampling simulations and WHAM. In particular, we found that the difference between the dissociation energies of MWT and MD4 in POPG was in quantitative agreement with the energy of the extra hydrogen bonds in MWT, due to its higher helical content than MD4. Finally, the absence of a PMF well near the surface of the POPC bilayer in case of MD4 was in agreement with the experimental finding [3] and our free MD simulations that MD4 does not bind to POPC.

The work and results presented in this thesis can be extended along several directions. First, it should be possible to extend the multiple detachment pathways model [1] to interpret intermediate rupture events recorded in the full retraction force time series, $F(t)$, thus gaining further insight into the complexity of peptide-lipid membrane interaction. In the framework of our theoretical approach, intermediate rupture events should be regarded as detachments of intermediate residues from the lipid membrane. Second, AFM force spectroscopy data of MWT and MD4 in POPC and E. coli membranes should be use to further elucidate the role of helical secondary structure in the occurrence of double (or even multiple) rupture events. Finally, a systematic MD simulation study that mimics single molecule, 
constant velocity AFM force spectroscopy experiments could provide a better understanding of the microscopic mechanisms involved in peptide-lipid membrane interactions. 


\section{Appendix A}

\section{Peptide-lipid affinity assay that reveals an interplay between solution structure and partitioning}

\section{A.1 Methods}

The three constructs exhibited distinct signatures in force spectra and significant differences in membrane activity (i.e., the probability of partitioning into the membrane). The partitioning measurements were corroborated with solution structures, as determined via MD simulations. Taken together, this work engenders confidence in a single-molecule peptidelipid bilayer affinity assay and provides novel characterization of a peptide-lipid interaction related to the activity of an important peripheral membrane protein.

Three geometrically distinct constructs were made: (a) single copy SecA2-11, LIKLLTKVFGC, (b) series, LIKLLTKVFG-GGSGG-LIKLLTKVFG-C, and (c) parallel, $2 \times$ [LIKLLTKVFGGG]-K-C (shown in Fig. A.1). A cystine (C) residue was added to the C-terminus of 

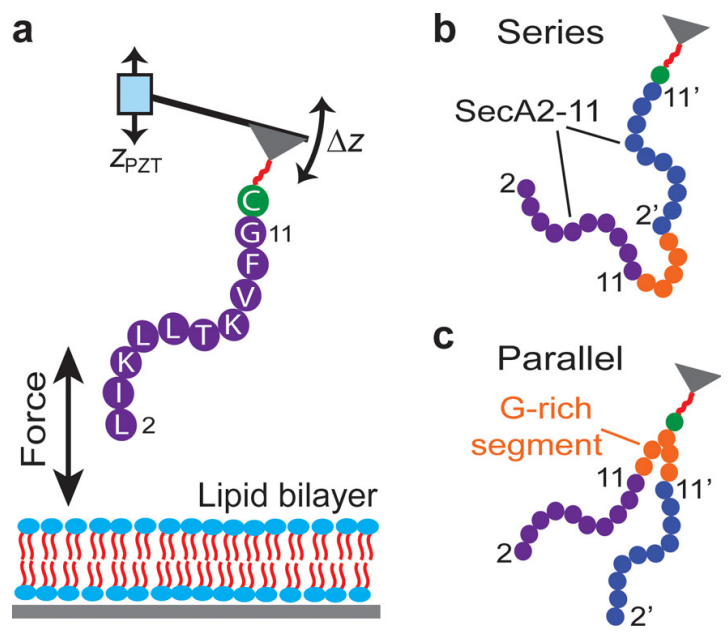

Figure A.1: (a) Single copy SecA2-11 (purple) covalently affixed to the AFM tip through a flexible PEG linker (red, not to scale). Sketches of (b) two copies of SecA2-11 linked in series and (c) two copies of SecA2-11 linked in parallel. Dual-copy sequences were separated by glycine-rich segments (orange); primed notation (2'-11', blue) identifies the copy nearest the tip for the series construct and the isopeptide-bonded branch for the parallel construct.

SecA2-11 [2] to closely mimic the experimental system, in which the peptide is covalently bonded through cysteine residue to a PEG linker attached to a functionalized AFM tip.

All atom models of these three constructs, including glycine-rich linkers, were built from the primary structure by using the Molefacture plugin in the VMD [37] molecular visualization and modeling software. Building the parallel SecA2-11 system required the insertion of an unconventional isopeptide bond (at the branching point), formed between the carboxyl terminus of G12' and the amino group of the side chain of the branched K13 residue (more in Appendix B). For all three systems, PDB (protein data bank) files with the atomic coordinates and protein structure (PSF) files were generated with the PSFGEN plugin in VMD. Next, by using the Solvate plugin of VMD, the three protein systems were solvated with TIP3P water molecules, which were pre-equilibrated under constant temperature $(T=300 \mathrm{~K})$ and pressure $(p=1 \mathrm{~atm})$. Each simulation box was made sufficiently large to avoid self-interaction of the peptide with its own images during the simulations 
under periodic boundary conditions. The final single copy, series, and parallel systems contained, respectively, a total of 10350 (3373), 33289 (10914), and 58109 (19160) atoms. To mimic the physiological ionic strength of the solution used in the AFM experiments $(300 \mathrm{mM})$, total of $19(21), 109(113)$, and $62(67) \mathrm{K}^{+}\left(\mathrm{Cl}^{-}\right)$ions were added to the three systems. For these purposes, Autoionize plugin in VMD was employed. In each case, the two extra anions were needed to neutralize the $+2 e$ charge of SecA2-11.

To eliminate bad contacts, the SecA2-11 systems were energy minimized and then equilibrated for several tens of nanoseconds using the molecular dynamics program NAMD 2.9 [41] with the CHARMM36 force field $[42,43]$. The simulations were carried out under normal temperature and pressure (as stated above for pre-equilibration). Periodic boundary conditions were used to reduce finite size effects. To mimic the experimental conditions, the alpha carbon $\left(C_{\alpha}\right)$ of the cysteine residue was harmonically restrained $\left(k=7 \mathrm{kcal} / \mathrm{mol} / \AA^{2}\right)$, as this end is connected to the PEG linker, which is in turn covalently affixed to the AFM tip. Van der Waals interactions were truncated at the cutoff distance of $12 \AA$ with a smooth switching function starting at $10 \AA$. Long-range electrostatic interactions were computed using the particle mesh Ewald method [47] with a grid spacing of $1 \AA$. The MD equations of motion were integrated with a multiple time step algorithm: 1 fs for bonding interactions, 2 fs for nonbonding interactions, and $4 \mathrm{fs}$ for electrostatic interactions. Constant temperature was maintained by coupling the system to a Langevin thermostat with a coupling coefficient of $1 \mathrm{ps}^{-1}$. The pressure was kept constant by using the Nosé-Hoover Langevin piston method [106] with a decay period of $100 \mathrm{fs}$ and a damping timescale of 50 fs. Following equilibration, a $30 \mathrm{~ns}$ long MD production run was carried out for each system. The coordinates of all atoms were saved every 10 ps and subsequently used to study the conformational dynamics of the SecA2-11 peptides. The MD simulations were carried out on 48 
Haswell cores with a performance of approximately $5 \mathrm{~ns} /$ day.

\section{A.2 Results and Discussion}

Activity, as obtained from an AFM experiment, is defined as a ratio of the number of events, $N_{e}$ and total number of attempts, $N_{a}$. Experimental results show that activity is 12,7 and $33 \%$ for single, series and parallel construct respectively. The observed difference in the activity for different constructs is significant and somewhere unexpected. It only seems counterintuitive for activity of series construct to be almost 2 and 5 fold smaller than for the single and parallel, respectively. This was the motivation behind the hypothesis that intra-peptide interactions between repeated sequences in the series and parallel constructs actually compete with the lipid bilayer, which could furthermore justify the observed differences in partitioning activity.

To determine where the decreased activity in the case of serial dimer comes from, MD simulations were employed and their results analyzed (Fig. A.2). As one way to quantify the conformational differences between parallel and series construct, time evolution and statistics of the angle $\theta$, between the axes of the two peptides were calculated (Fig. A.2a-c). The peak position in the distribution function $P(\theta)$ for the series construct is considerably smaller than that for the parallel, indicating that the two SecA2-11 monomers in the series system tend to attract each other, whereas, in the parallel case, they repel. The dominant orientation for the parallel peptide is splayed outward (Fig. A.2b), with a $\sim 100^{\circ}$ angle between the repeated SecA2-11 sequences, stabilized by hydrogen bonding. With both $\mathrm{N}$-termini available, this splayed geometry is poised to interact with the bilayer surface significantly more than the compacted series construct (Fig. A.2c). There might be additional factors 
that are likely contributing to the observed variations in the membrane activity.

To then express the degree of compactness of a single SecA2-11 peptide, radius of gyration of the $(N=10) C_{\alpha}$ atoms was calculated as $R_{g}=\sqrt{\sum_{i}\left(r_{i}-r_{c}\right)^{2} / N}$, where $r_{i}$ is the position vector of the $i$-th $C_{\alpha}$ (at a given time) and $r_{c}$ is the corresponding centroid. The small standard deviations $(<1 \AA$ ) of the radius of gyration for each SecA2-11 peptide imply that the systems were well-equilibrated. As the results for the $R_{g}$ show (Fig A.3), the series forms a more compact structure than the parallel, also in the agreement with the $P(\theta)$ results (Fig. A.3).

We believe that, to a great extent, membrane affinity of SecA2-11 is likely due to the hydrophobic leucine residues located at the extreme N-terminus (positions 5 and 6). Taken together, these three residues constitute $30 \%$ of the core SecA2-11 sequence. To see if this assumption is in agreement with previously obtained results, the freely accessible surface area (SASA) [107] of this lipophilic residue was evaluated. The results showed $>25 \%$ enhancement in the accessibility of leucine for the parallel construct compared with the series (Fig. A.4). Therefore, factors including a less compact, splayed orientation, and greater accessibility of hydrophobic residues impart significant advantages upon the parallel construct for partitioning when compared with the series construct. To summarize, MD simulations revealed that both the series and parallel constructs interacted strongly with themselves; however, the consequences of the intra-peptide interactions on the conformations were distinct and provide a molecular-level justification for the experimental observations. 


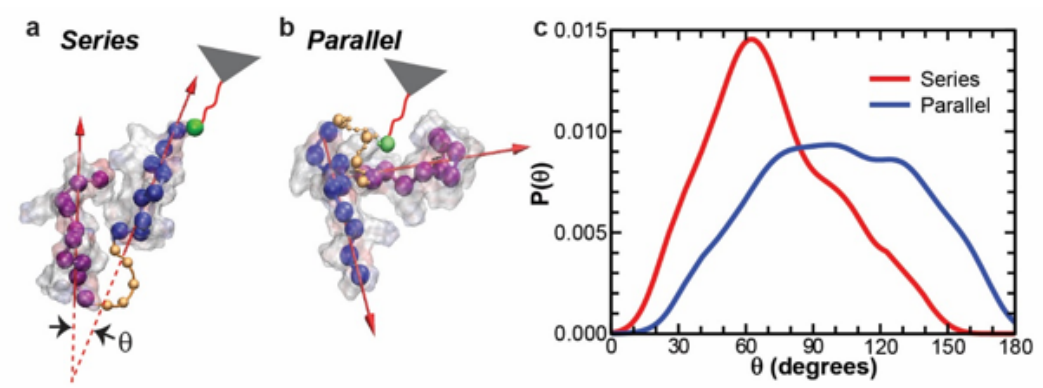

\begin{tabular}{|c|c|c|c|c|c|c|}
\hline & Residue & $\begin{array}{c}\text { Radius of } \\
\text { gyration }[\AA \AA]\end{array}$ & \multicolumn{2}{|c|}{$\begin{array}{l}\text { H-bonding } \\
\text { [\% occupied] }\end{array}$} & $\begin{array}{l}\text { Leu accessibility } \\
\text { to solvent }\left[\AA^{2}\right]\end{array}$ & $\theta$ [deg] \\
\hline Single & $2-11$ & $6.7 \pm 0.8$ & L5 $\cdots$ T7 & $4 \%$ & $490 \pm 31$ & \\
\hline \multirow{3}{*}{ Series } & $2-11$ & $6.9 \pm 0.4$ & $\begin{array}{r}\mathrm{K} 8^{\prime} \cdots \mathrm{L} 2 \\
\mathrm{~L} 2 \cdots \mathrm{T} 7\end{array}$ & $\begin{array}{l}21 \% \\
5 \%\end{array}$ & $379 \pm 44$ & \multirow{3}{*}{$72 \pm 29$} \\
\hline & $2^{\prime}-11^{\prime}$ & $6.6 \pm 0.8$ & T7... L2 & $1 \%$ & $396 \pm 78$ & \\
\hline & All & $9.9 \pm 1.7$ & \multicolumn{2}{|c|}{$31 \%$} & $775 \pm 105$ & \\
\hline \multirow{3}{*}{ Parallel } & $2-11$ & $7.3 \pm 0.9$ & K8' ...G11 & $20 \%$ & $501 \pm 46$ & \multirow{3}{*}{$101 \pm 35$} \\
\hline & $2^{\prime}-11^{\prime}$ & $6.8 \pm 0.5$ & $\mathrm{~L}^{\prime} \cdots \mathrm{G} 13$ & $13 \%$ & $480 \pm 38$ & \\
\hline & All & $12.0 \pm 2.2$ & \multicolumn{2}{|c|}{$62 \%$} & $981 \pm 60$ & \\
\hline
\end{tabular}

Figure A.2: Snapshots of (a) series and (b) parallel constructs in solution at room temperature (water has been removed for clarity). Unprimed residues are drawn purple, primed blue (as defined in Fig. A.1). The orientation of the repeated peptide sequences was characterized by the angle $\theta$ between the axes (red arrows) of the peptides. (c) Comparison between the probability distributions $P(\theta)$ for the two constructs indicating that the parallel construct is more open, in general, whereas the series is more compact and folded upon itself. Table: parameters calculated from the MD simulations. For series and parallel constructs, quantities were calculated for each copy of SecA2-11, as well as for the complete peptide.

\section{A.3 Conclusion}

Using series and parallel peptides with different geometries, but near identical chemical composition, provided a means to isolate the role of the peptide structure in partitioning. MD simulations revealed solution structures that would clearly modify the membrane activity and do so in a manner consistent with the experimental results, which showed an ap- 

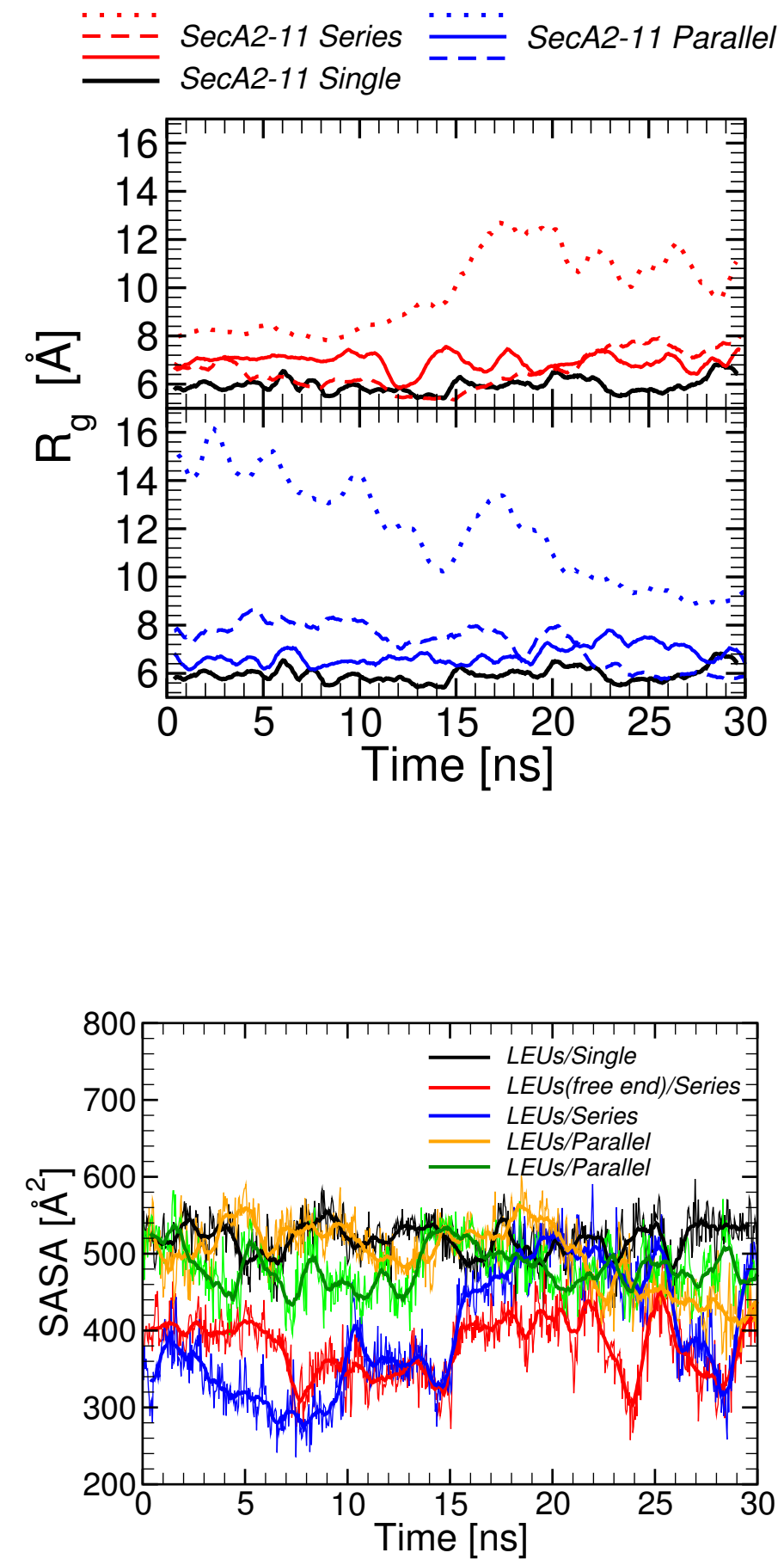

Figure A.3: The radius of gyration $\left(R_{g}\right)$ of the $C_{\alpha}$ atoms of SecA2-11 in the series (top panel, red) and parallel (bottom panel, blue) constructs plotted versus time. Solid and dashed curves correspond to the two SecA2-11 monomers in the series (red) and parallel (blue) systems, and show similar behavior to $R_{g}$ for the single copy system (black, shown in both panels). In contrast, $R_{g}$ for the entire series (parallel) construct, shown as dotted red (blue) curve, exhibits significant variation in time, indicative of noticeable conformational changes as described in this chapter.

Figure A.4: Time evolution of SASA for each construct, averaged for all three leucines (LEUs). Black line represents single copy, red represents SecA2-11 copy from the series construct and located at the free end of peptide, while the blue represents the other copy (closer to the cystine). Copies constituting parallel geometry are colored in green and yellow. 
proximately 5-fold enhancement in bilayer association probability for the parallel peptide compared with the series. In summary, we united high-precision single-molecule methods with analytical modeling, computational simulations, and bulk biochemical techniques and thus characterized a peptide-lipid bilayer interaction related to the mode of action of a model peripheral membrane protein. More generally, our work provides a framework to advance the understanding of other protein-lipid interactions, including with biologically relevant lipid mixtures. 


\section{Appendix B}

\section{Isopeptide bond}

Building the parallel SecA2-11 construct (described in Ch. 3) required a patch in PSFgen to build an unconventional isopeptide bond, which is formed at the branching point, between the carboxyl terminus of G13 and the amino group of the sidechain of the branched K residue. Thus, we were able to use CHARMM36 peptide bond parameters.

\section{B.1 Lysine and default peptide bond topology entry}

In the Fig. B.1, RESI statement indicates that LYS is the residue name with the total charge of +1.00 . All of the atoms in the residue (starting with the ATOM statements) are listed next. Each atom has a name (e.g. N, HN, CA) and the type (e.g. NH1, H, CT1), and combination of these two is unique for each atom in the system. The latter is especially important for taking the parameters from the parameter file (while running a NAMD simulation). Next to the each atom, partial charge is listed (e.g. -0.47, 0.31, 0.07). The GROUP statements are grouping the atoms into integer-charge groups, which is mostly done for clarity, 
but

are

are

not

used

by

NAMD.

Connectivity between atoms is listed next. Each BOND statement followed by a list of pairs of atoms to be connected with bonds (the DOUBLE statement states if there is a double bond, but it does not affect the resulting PSF file). By the default, atom $\mathrm{C}$ is bonded to $+\mathrm{N}$, where " + " implies the following residue. Similarly, a bond between $\mathrm{N}$ and $-\mathrm{C}$ indicates that $-\mathrm{C}$ is the preceding residue. Order of atoms within a bond or the order of bonds itself is not significant. The angle and dihedral terms are autogenerated and therefore, do not have to be specified (same applies to IC, i.e. internal coordinates). If auto-generated angles are not optimal, that will be corrected during the energy minimization and later, from by reading the correct values from parameter

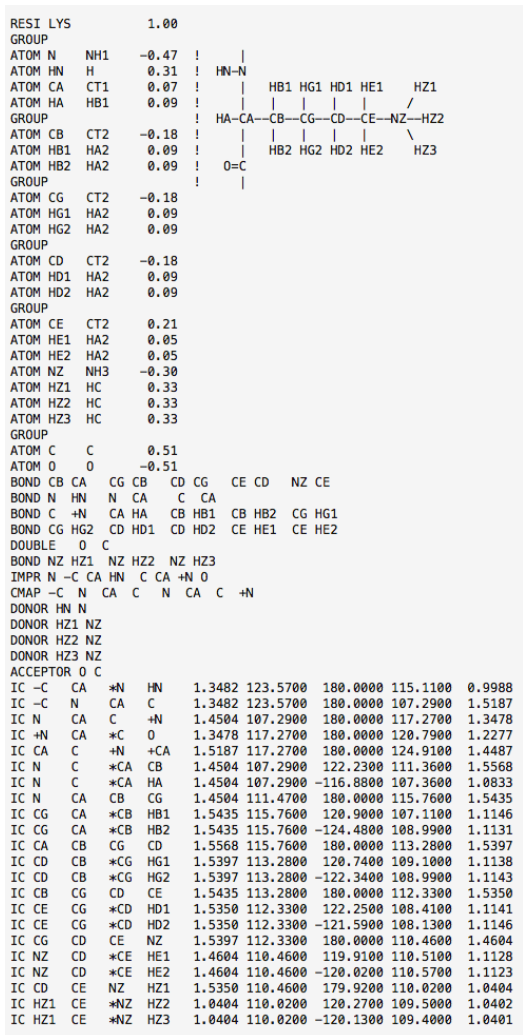
file. IMPR, i.e., less common improper dihedrals, must be listed explicitly. In this case, there are two impropers, that maintain the planarity of the peptide bond and thus protein backbone. Each improper is represented by sets of four atoms and atom that is listed first is the one to which other three are bonded. The DONOR and ACCEPTOR statements specify pairs of atoms eligible to form hydrogen bonds, but note that these will not be included in PSF file and are ignored by psfgen module. Internal coordinates (IC) come in the following form $I C A B C D$ [bond $(A B)]$ [angle(ABC)] [dihedral $(A B C D)][a n-$ $\operatorname{gle}(B C D)]$ [bond $(C D)]$. If the third atom is marked with asterisk (i.e. ${ }^{*} C$ ), that means 
that dihedral is an improper and marked atom $(C$, in this example) is a chiral center. Peptide bond topology entry (Fig. B.2) only states atoms are to be bonded (C atom from previous residue and $\mathrm{N}$ atom from following residue) and planarity of peptide bond. These two entries were used as starting point for building the isopeptide patch.

\section{B.2 Isopeptide bond patch}

Isopeptide bond (IPB)

has the same (neutral)

charge, as does the pep-

tide bond. Next, how

backbone $\mathrm{N}$ that partici-

pates in peptide bond is

only bonded to $-\mathrm{C}$ and

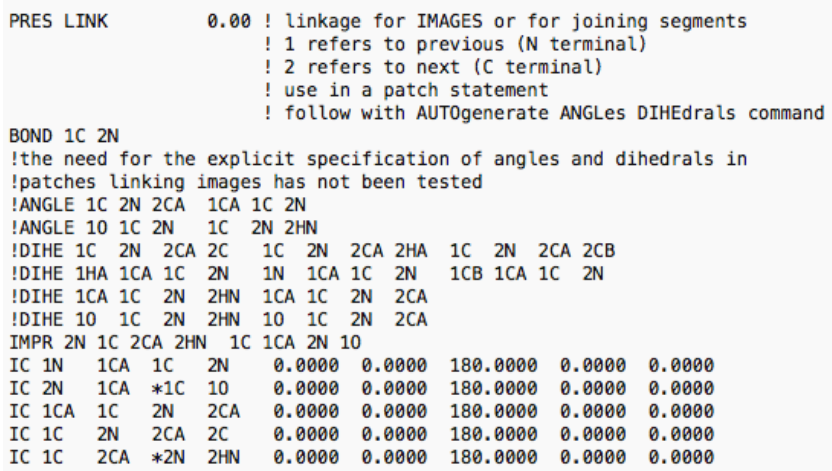

Figure B.2: Topology entry for peptide bond one $\mathrm{H}$ atom, in the same manner, we need to delete 2 "extra" $\mathrm{H}$ atoms that are bonded to $\mathrm{N}$ at the end of sidechain that will now be bonded to C-terminal of G12'. Following is the reassignment of charge, so that isopeptide bond maintains neutral. This is done by employing logic and algebra (otherwise, we would need to use Gaussian or a similar program). Then the glycine's default impropers need to be deleted and redefined in such way so $\mathrm{N}(\mathrm{CA})$ is replaced by NZ (CE) atom. Zoomed-in isopeptide bond is shown in Fig. B.4. 


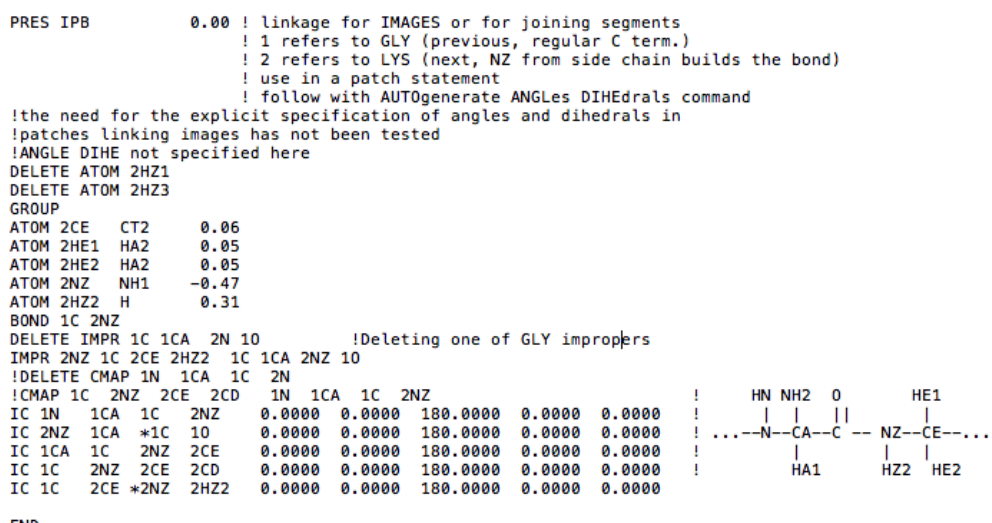

Figure B.3: Script for isopepeptide bond (IPB)

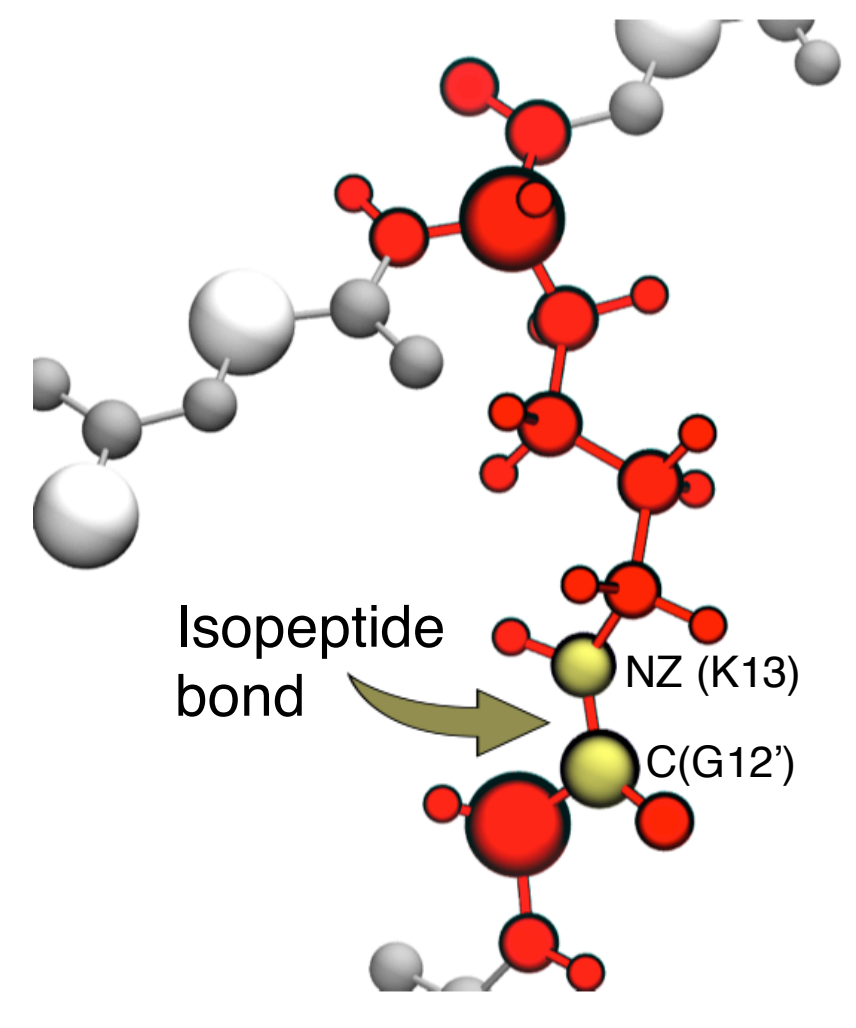

Figure B.4: CPK representation was used for all atom shown (except for $C_{\alpha}$, which are shown with vdW spheres). G12' and K13 residues are colored in red, in contrast to remainder of peptide (colored in silver/white). NZ (K13) and $\mathrm{C}(\mathrm{G} 12$ ') atoms are further emphasized by using color $\tan$. 


\section{Appendix C}

\section{Umbrella Sampling \& WHAM}

To computationally analyze a system means to determine the free energy changes along the reaction coordinate(s) (RC). Umbrella sampling is a standard and widely used method for calculating the potential of a mean force (PMF). It uses harmonic biasing potentials in order to sample the structural conformations along the RC, divided into windows, in a given time. With enough (overlapping) windows, PMF can be calculated by properly combining the data from each window. Common way to do so is by employing weighted histogram analysis method (WHAM). I will now focus on some technical details, so some of the future student scan use this appendix.

The collective variables module (colvars), a software that interfaces NAMD, can be used for high performance umbrella sampling. Each window can be sampled in separate simulation or during longer, unified simulation by specifying number of stages/windows (numStages), that is otherwise considered to be one (single window). Following is the segment of colvars input file that refers to this and everything that is after \# is a comment:

harmonic \{ \#applying harmonic potential 


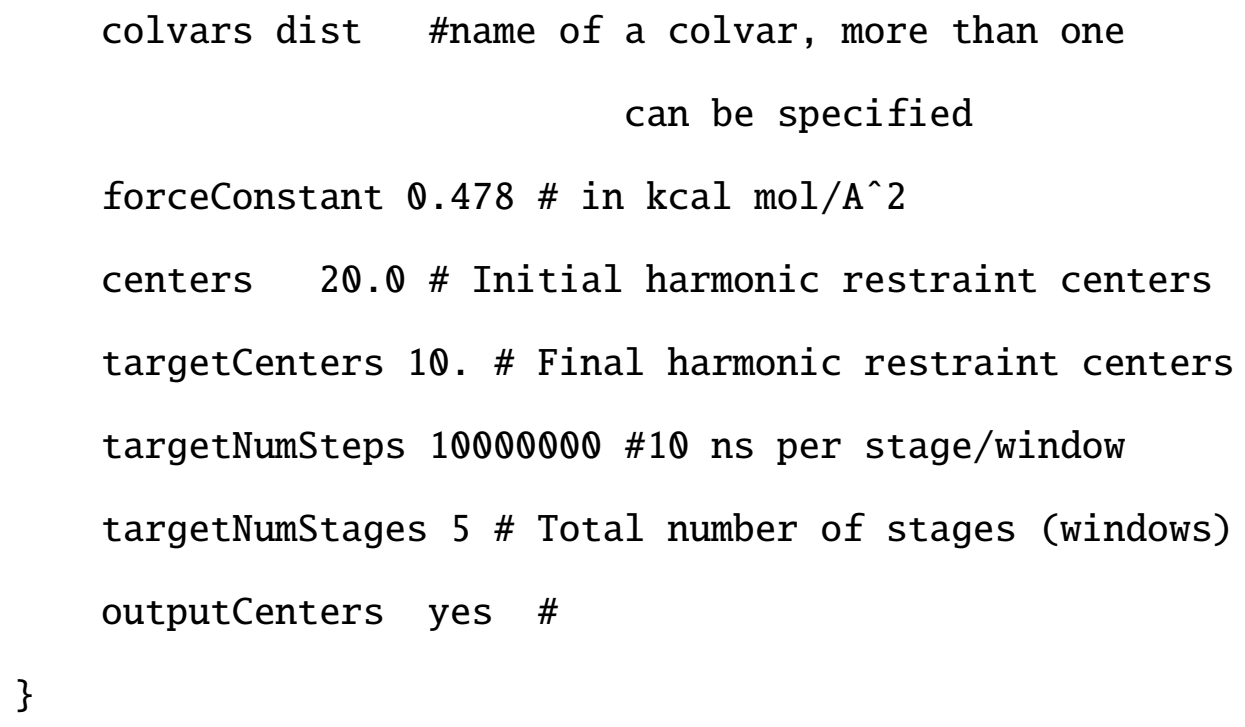

It is important that in configuration file number of steps is set as (targetNumStages +1$) \times$ targetNumSteps, because that way both first and last window will be sampled.

I will focus here mainly on colvar $\operatorname{dist} Z$ ( $\mathrm{Z}$ or whatever the direction perpendicular to bilayer surface is). It is commonly used in protein-lipid bilayer systems, where relative distance between the COM of a protein (or one of it's relevant segments) and COM of lipid bilayer is good choice of RC.

\section{Questions that need to be answered when running US sim- ulation}

1. How long should each window be sampled?

2. What should be the spacing between adjacent window centers?

3. What should be the umbrella potential (i.e. spring constant)?

4. How often data point should be saved? 
5. Is specifying the width (of the colvar grid) important and what value should be used?

Anywhere from 5 to 15 ns (i.e. 15000000 steps) is a good starting point, depending on a complexity of a system. Once the targetNumSteps is set, after only a few windows are sampled, one should check (i) if $\mathrm{P}\left(\mathrm{z}_{i}, \mathrm{t}_{n}\right)$ and $\mathrm{P}\left(\mathrm{z}_{i}, \mathrm{t}_{n+1}\right)$ are converging (here, $\mathrm{z}_{i} \equiv \mathrm{RC}$ value in the $i$-th window) and (ii) if there is enough overlapping between the windows. For example, PMF of protein going inside/outside lipid bilayer might even require more than $100 \mathrm{~ns}$ in order to accomplish proper sampling. Along RC, adjacent windows doesn't necessarily need to be equidistant. Some segments might need more sampling whereas others doesn't. Once $\mathrm{P}\left(\mathrm{x}_{i}\right)$ is plotted, one should conclude if the distance used was enough. In case when $\mathrm{RC}$ is a distance, $2 \AA$ is a good starting point. It is also important to note that distance between centers and value of forceConstant are closely related and inversely proportional, i.e. if center are more apart, then umbrella potential should have lower value (more about this in the next section). As it was mentioned above, selecting spring constant for harmonic potential is not independent of distance between the windows. In literature, anything from $200 \mathrm{~kJ} \mathrm{~mol}^{-1} \mathrm{~nm}^{-2}$ (i.e. $\left.0.478 \mathrm{kcalmol}^{-1} \AA^{-2}\right)$ to $3000 \mathrm{~kJ} \mathrm{~mol}^{-1} \mathrm{~nm}^{-2}\left(7.17 \mathrm{kcal} \mathrm{mol}^{-1} \AA^{-2}\right)$ is accepted. Once the analysis of the saved data is done, one can conclude whether used kvalue is too loose or stiff. It is always a possibility that different windows require different values of $\mathrm{k}$. Acceptable standard deviation (std) of data in windows that are separated $1 \AA$ is $0.3-0.5 \AA$, otherwise windows are not separated enough. Often reviewers ask why that exact k-value was used, and unless there is previous work that was used as reference, only good answer would be "we have tried different values and this one worked the best". Another thing one needs to check is if the colvar outputs for each time step are independent. By setting colvarsTrajFrequency to 10000 (output every $10 \mathrm{ps}$ ), this is successfully avoided. I actually output every $1 \mathrm{ps}$, and then, with a simple bash command, I can extract only every 
10th line from the *.traj file (awk 'NR\%10==0'*.traj).Then, if i need more data points, I can use them, which is not the case when they are saved less frequently. It can be found in some tutorials that for good sampling of RC via colvars, one need to set $w$ (idth) (parameter that represents fluctuation) to 0.1 , that is otherwise considered to be 1 ( $w$ has the same physical unit as the colvar value and hence defines an effective colvar unit). I have tested this and found it not to be relevant. I maintained similar to same results with various wvalues.

\section{Applying WHAM}

Once the sampling simulation(s) has finished, it is time to apply WHAM. Line in Terminal window that does that (without the Bootstrapping error estimate method) is:

wham min max numBins tolerance temperature $\theta$ metadataInput output_pmf

Bash/tcl scripts below automate this process. (They will be listed next, since the purpose of this appendix is to serve as a kind of "manual" for future students.)

First, the bash script extracts only certain columns and/or rows from *.traj file. In the following example, only last $8 \mathrm{~ns}$ of total of $10 \mathrm{~ns}$ per each window are saved. Then, arithmetic mean of two colvars will be saved to the metadata file:

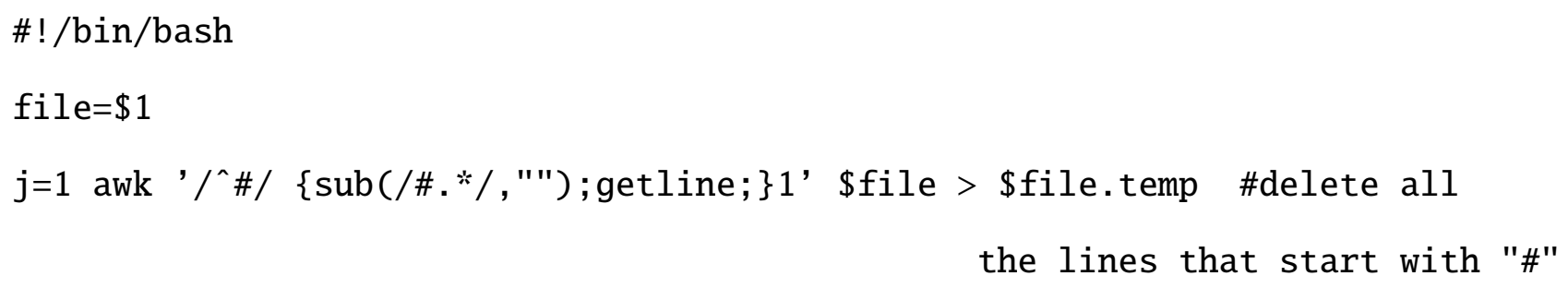




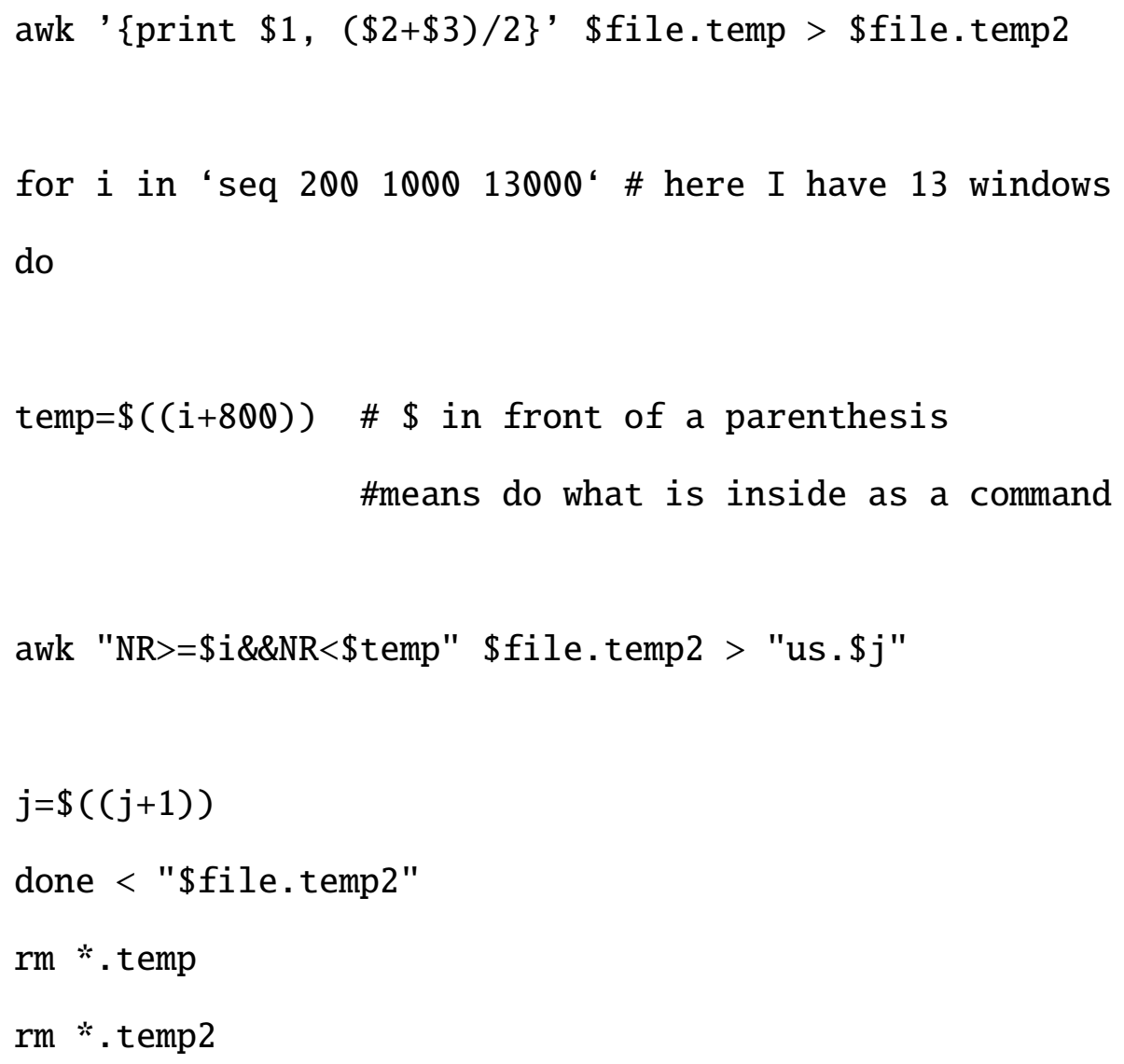

Next, metadata file is created, named INPUT with tcl script, that can also be ran from Terminal window (tclsh):

set input [open "INPUT" w]

for $\{$ set $j 1\}\{\$ j<=13\}$ incr $j\}\{$

puts \$input "us.\$j[expr $-9+\$ j] 5 . " \#$ first center is at -8 and $\mathrm{k}=5$ \} 


\section{close \$input}

Finding minimum and maximum value can be done in many ways. one way is by using awk in bash:

awk ' $\$ 2>\max \{\max =\$ 2 ;\} ; \operatorname{END}\{$ print $\max \}$ ' file \#refers to 2 . column awk 'min=="" || $\$ 2<\min \{\min =\$ 2 ;\} ; \operatorname{END}\{$ print $\min \}$ ' file

The only two things that haven't been mentioned yet are (i) tolerance and (ii) number of bins. Optimal value for tolerance is $10^{-4}$ and rule of thumb for selecting number of bins is $\frac{\left|z_{i}-z_{f}\right|}{s t d}$. I want to explicitly state here that any of these number nor any numbers found in previous publications/tutorials should be taken blindly. This should merely serve as a guideline. 


\section{Appendix D}

\section{MD test runs of MWT and MD4}

Prior to MD simulations whose results were analyzed in Ch. 5, series of simulation in which MWT (MD4) had net charge of $+5 e$ were completed and will be analyzed here. They had default $\mathrm{N}$ - and $\mathrm{C}$-terminus, hence the reduced net charge. They will be referred to as MWT' (MD4'). Figs. D.1 and D.3 show results that are in principle very similar to the ones in Ch.5. Namely, (i) single copy of MWT' partitioned the bilayer, whereas MD4' never did (even though it seemed, around 300 and 400 ns, that bottom copy will might partition, this was never stabilized), and then, (ii) both copies of MWT' and MD4' strongly interacted with the POPG bilayer. Same applies to the helical content, shown in Figs. D.2 and D.4. Top (bottom) MWT' had helical content above 70\% (60\%), whereas top MD4' had helical content below $20 \%$ and bottom copy was a random coil during last $100 \mathrm{~ns}$ of free simulation time.

Additionally, simulation with single copy of MWT in POPC was simulated in aim to observe spontaneous insertion into the bilayer, i.e., without any potential influence coming from the second copy. This is exactly what analysis showed (Fig. D.5). On a 50 ns time 

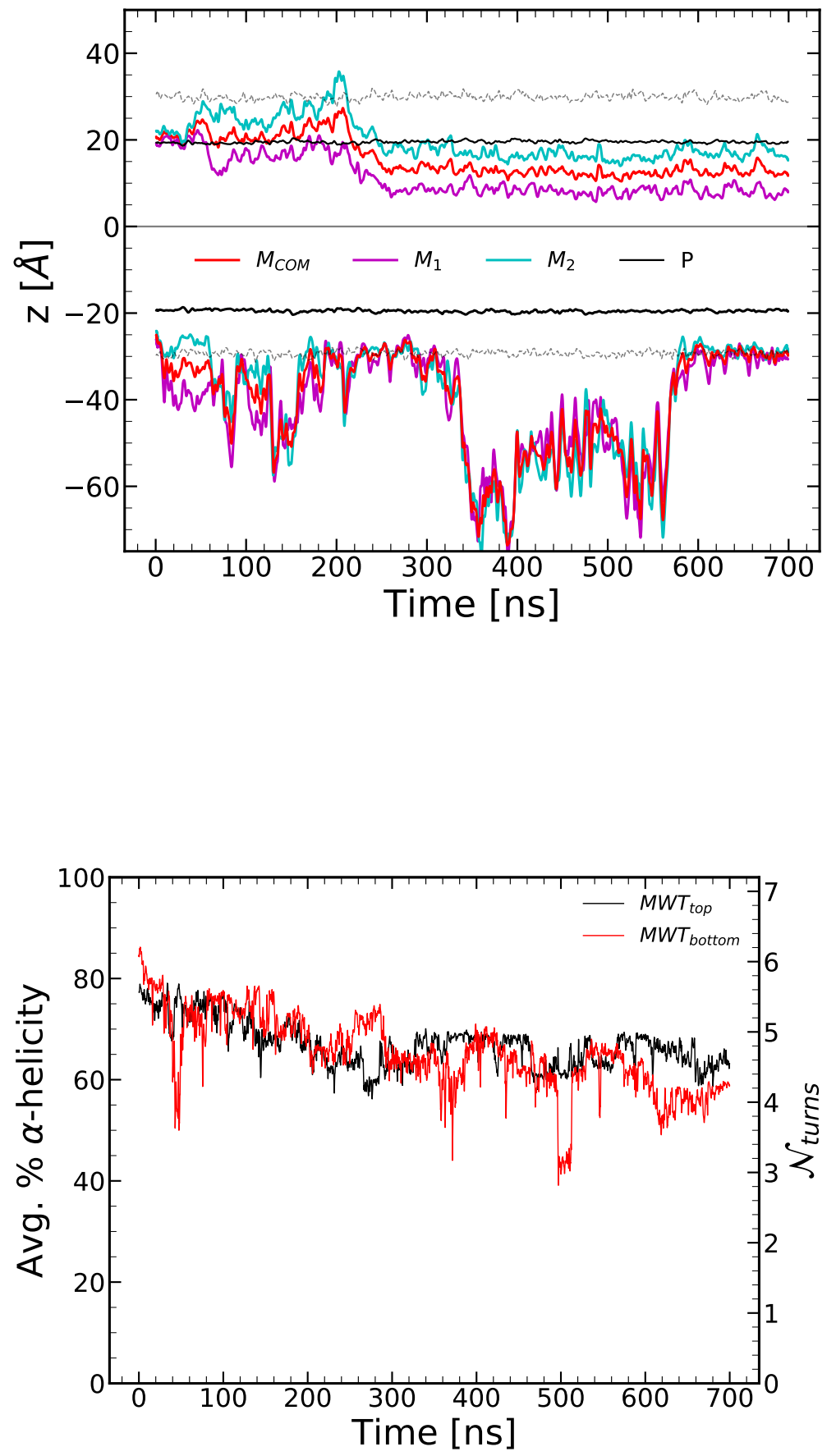

Figure D.1: Time evolution of $z$-coordinate for MWT' in POPC. COM of peptide is shown in red, whereas COM of SEG1 and SEG2 are shown in magenta and cyan, respectively. Phosphate centers $(\mathrm{P})$ are represented with black line and gray dashed line illustrates maximum (minimum) $z$-coordinate of all lipid atoms in the top (bottom) leaflet.

Figure D.2: Time evolution of helical content for MWT' in POPC. Red line refers to the bottom copy and black to the top copy of MWT'. Peptides lost some helical content over total simulation time (note that SEG2 remained fully folded in both cases). 

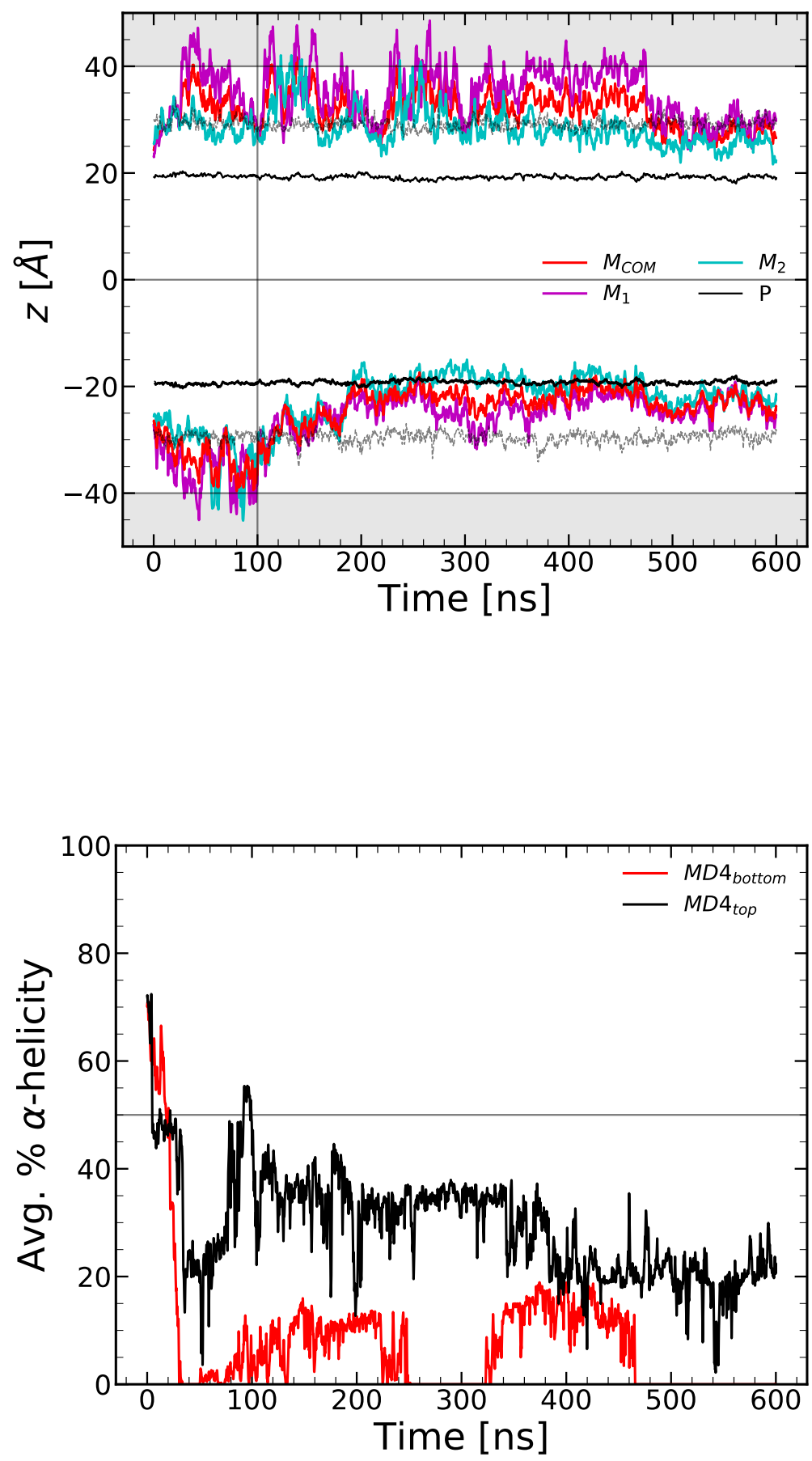

Figure D.3: Time evolution of $z$-coordinate of MD4' in POPC. COM of peptide is shown in red, whereas COM of SEG1 and SEG2 are shown in magenta and cyan, respectively. Phosphate centers $(\mathrm{P})$ are represented with black line and gray dashed line illustrates maximum $z$-coordinate of all lipid atoms in the upper leaflet. Shaded regions represent the forbidden zones, i.e., if COM of a peptide crosses relative distance of $z= \pm 2 \mathrm{~nm}$ from the phosphate centers, harmonic potential was applied.

Figure D.4: Time evolution of helical content for MD4' in POPC. Black line represents top, and red the bottom copy of MD4', which loses helical structure completely. Gray line is plotted only for the reference. 


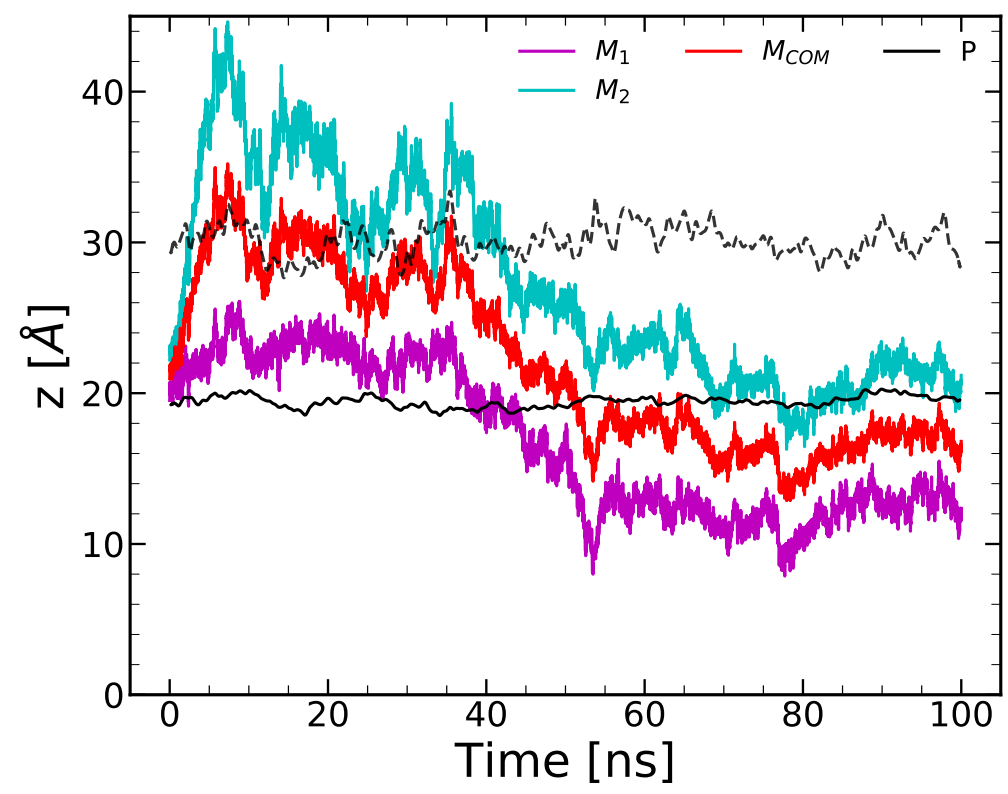

Figure D.5: Time evolution of $z$-coordinate of a single MWT copy in POPC. COM of peptide is shown in red, whereas COM of SEG1 and SEG2 are shown in magenta and cyan, respectively. Phosphate centers (P) are represented with black line and gray dashed line illustrates maximum $z$-coordinate of all lipid atoms in the upper leaflet.

scale, single copy of MWT partitioned the POPC bilayer and $\Delta z$ had not changed in the next 100 ns (standard deviation was below $2 \AA$ ). We believe this has something to do with electrostatic repulsion, how each copy carries $+6 e$ charge and the bilayer is neutral overall. 


\section{Appendix E}

\section{Analysis of hydrogen bonds formed between bilayers (POPG/POPC) and peptides (MWT and MD4)}

Some of the previously published studies suggest that hydrogen bonds provide an efficient mechanism of long-distance protein-lipid coupling [108] and when formed between charged residues of a peptide and phosphate in a lipid bilayer, they stabilize the proteinlipid interaction [109]. By interpreting the results in a similar manner, in case of PC systems, hydrogen bonds formed between peptide and lipid bilayer were one more way to quantify the peptide-lipid interaction. With 5-fold higher number of hydrogen bonds in case of MWT-PC, this was yet another indicator of the difference between MWT and MD4 peptides (Fig. E.1). Furthermore, Fig. E.2 shows higher number of hydrogen bonds in PG systems than in case in PC, as one might expects. Analysis on residues that were participating in the hydrogen bonding can be completed, but is outside of scope of this thesis. END 


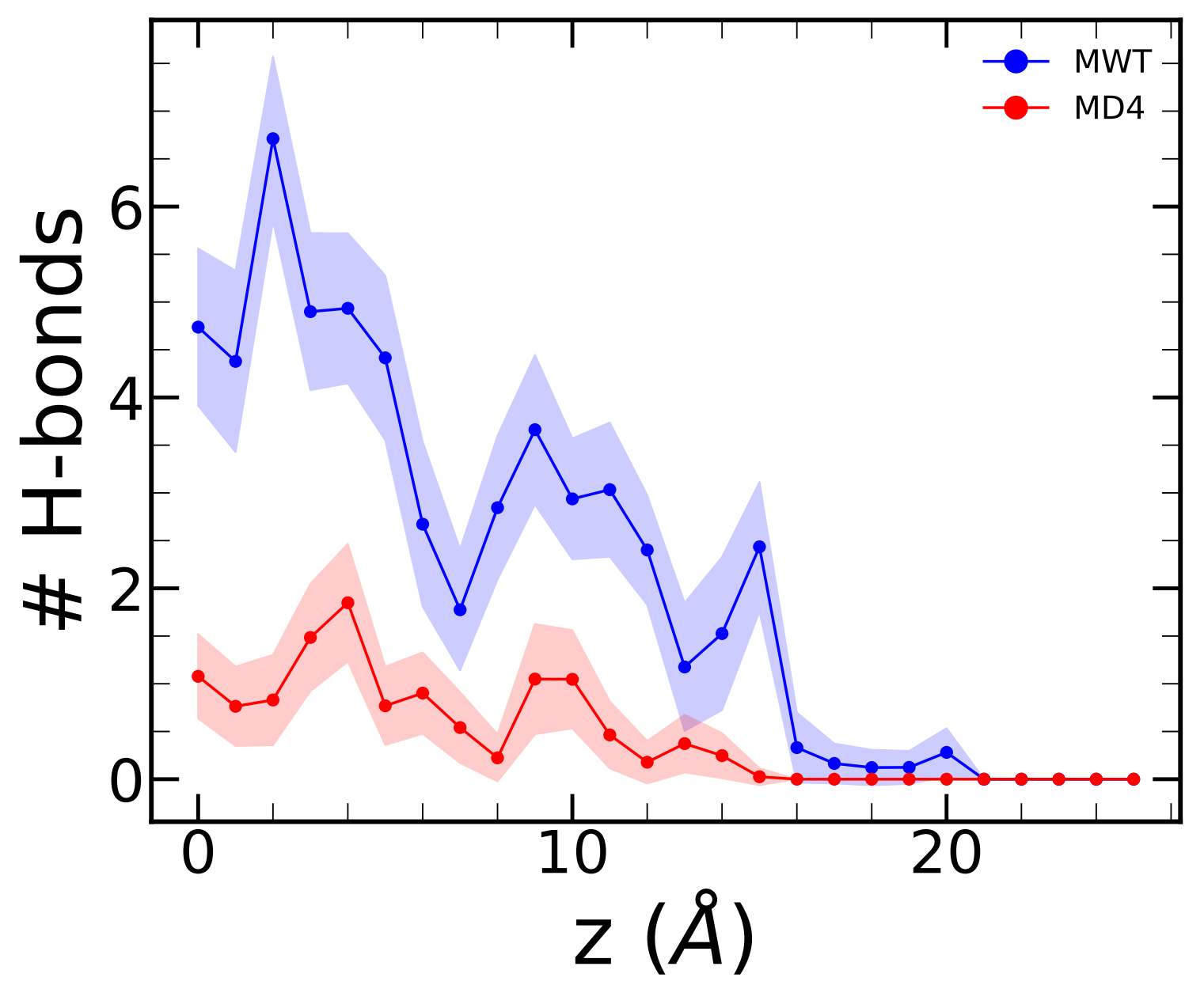

Figure E.1: Average number of hydrogen bonds, per window, formed between POPC bilayer and MD4 (red) and MWT (blue). 


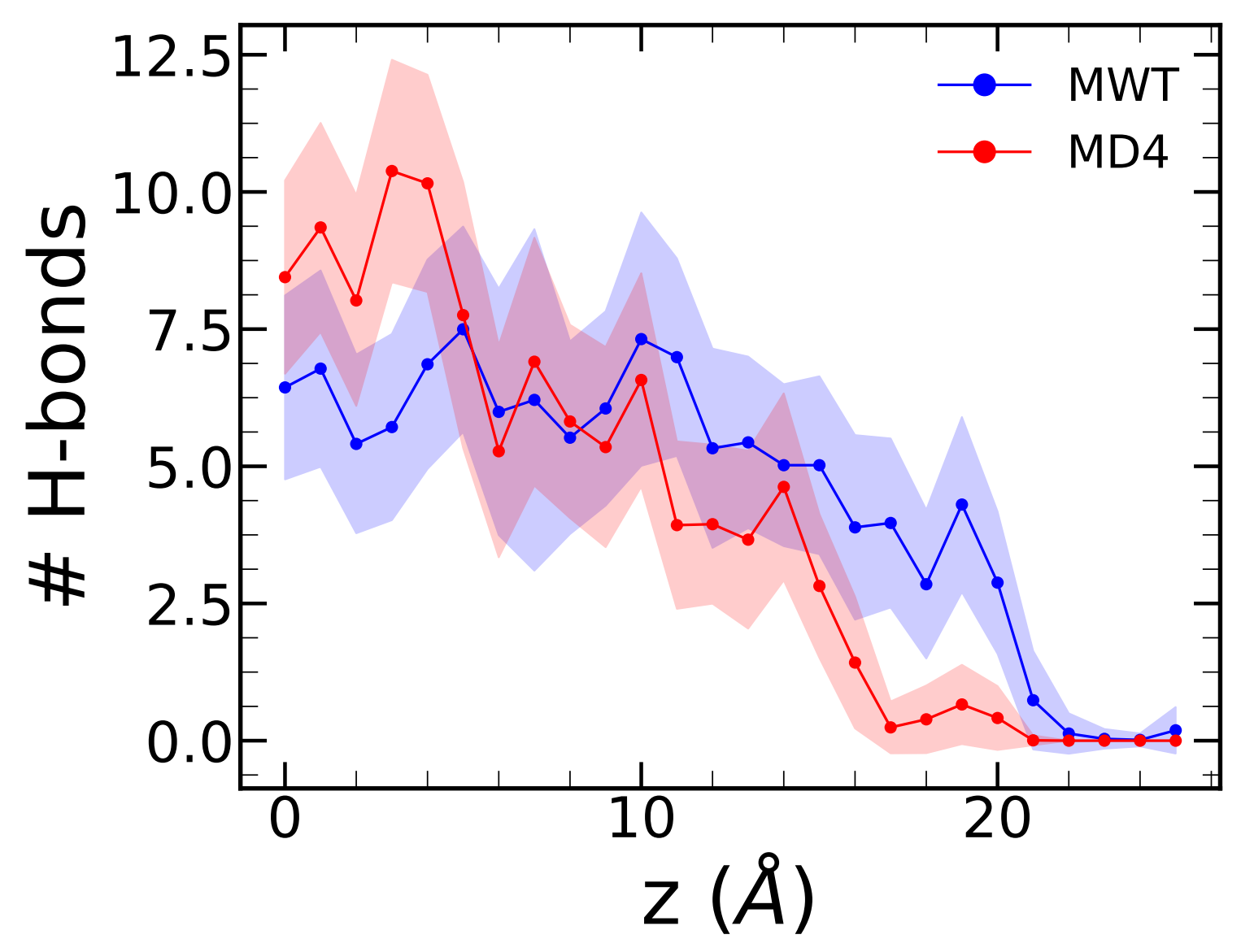

Figure E.2: Average number of hydrogen bonds, per window, formed between POPG bilayer and MD4 (red) and MWT (blue). 


\section{Bibliography}

[1] Milica Utjesanovic, Tina R. Matin, Krishna P. Sigdel, Gavin M. King, and Ioan Kosztin. Multiple stochastic pathways in forced peptide-lipid membrane detachment. Scientific Reports, 9(1), December 2019.

[2] Tina R. Matin, Krishna P. Sigdel, Milica Utjesanovic, Brendan P. Marsh, Fabio Gallazzi, Virginia F. Smith, Ioan Kosztin, and Gavin M. King. Single-Molecule PeptideLipid Affinity Assay Reveals Interplay between Solution Structure and Partitioning. Langmuir, 33(16):4057-4065, April 2017.

[3] Alexey S Ladokhin and Stephen H White. Folding of amphipathic $\alpha$-helices on membranes: Energetics of helix formation by melittin 1 1Edited by D. Rees. Journal of Molecular Biology, 285(4):1363-1369, January 1999.

[4] Peptide-Lipid Interactions, Volume 52 - 1st Edition.

[5] Rob Phillips, Tristan Ursell, Paul Wiggins, and Pierre Sens. Emerging roles for lipids in shaping membrane-protein function. Nature, 459(7245):379-385, May 2009. 
[6] Florian Cymer, Gunnar von Heijne, and Stephen H. White. Mechanisms of Integral Membrane Protein Insertion and Folding. Journal of Molecular Biology, 427(5):999-1022, March 2015.

[7] S.A. Simon and T.J. McIntosh. Peptide-Lipid Interactions, volume 52 of Current Topics in Membranes. Academic Press, 2002.

[8] N. Schwierz, S. Krysiak, T. Hugel, and M. Zacharias. Mechanism of reversible peptidebilayer attachment: Combined simulation and experimental single-molecule study. Langmuir, 32:810-821, 2016.

[9] G. Andre, R. Brasseur, and Y. F. Dufrene. Probing the interaction forces between hydrophobic peptides and supported lipid bilayers using afm. J Mol Recognit, 20(6):538-45, 2007.

[10] A. B. Churnside, R. M. Sullan, D. M. Nguyen, S. O. Case, M. S. Bull, G. M. King, and T. T. Perkins. Routine and timely sub-piconewton force stability and precision for biological applications of atomic force microscopy. Nano Lett, 12(7):3557-61, 2012.

[11] G. I. Bell. Models for the specific adhesion of cells to cells. Science, 200(4342):618627, May 1978.

[12] E. Evans and K. Ritchie. Dynamic strength of molecular adhesion bonds. Biophysical Journal, 72(4):1541-1555, April 1997.

[13] Gerhard Hummer and Attila Szabo. Kinetics from Nonequilibrium Single-Molecule Pulling Experiments. Biophysical Journal, 85(1):5-15, July 2003. 
[14] Olga K. Dudko, Gerhard Hummer, and Attila Szabo. Intrinsic Rates and Activation Free Energies from Single-Molecule Pulling Experiments. Phys. Rev. Lett., 96(10):108101-4, March 2006.

[15] Olga K. Dudko, Gerhard Hummer, and Attila Szabo. Theory, analysis, and interpretation of single-molecule force spectroscopy experiments. PNAS, 105(41):1575515760, October 2008.

[16] Ciro Cecconi, Elizabeth A. Shank, Carlos Bustamante, and Susan Marqusee. Direct Observation of the Three-State Folding of a Single Protein Molecule. Science, 309(5743):2057-2060, September 2005.

[17] Miklós S. Z. Kellermayer, Steven B. Smith, Henk L. Granzier, and Carlos Bustamante. Folding-Unfolding Transitions in Single Titin Molecules Characterized with Laser Tweezers. Science, 276(5315):1112-1116, May 1997.

[18] Piotr E. Marszalek, Hui Lu, Hongbin Li, Mariano Carrion-Vazquez, Andres F. Oberhauser, Klaus Schulten, and Julio M. Fernandez. Mechanical unfolding intermediates in titin modules. Nature, 402(6757):100-103, November 1999.

[19] M. Rief, M. Gautel, F. Oesterhelt, J. M. Fernandez, and H. E. Gaub. Reversible unfolding of individual titin immunoglobulin domains by afm. Science, 276(5315):1109-12, 1997.

[20] Michael Schlierf, Hongbin Li, and Julio M. Fernandez. The unfolding kinetics of ubiquitin captured with single-molecule force-clamp techniques. PNAS, 101(19):7299-7304, May 2004. 
[21] Michael Schlierf and Matthias Rief. Single-Molecule Unfolding Force Distributions Reveal a Funnel-Shaped Energy Landscape. Biophys J, 90(4):L33-L35, February 2006.

[22] William J. Greenleaf, Kirsten L. Frieda, Daniel A. N. Foster, Michael T. Woodside, and Steven M. Block. Direct Observation of Hierarchical Folding in Single Riboswitch Aptamers. Science, 319(5863):630-633, February 2008.

[23] Jan Liphardt, Bibiana Onoa, Steven B. Smith, Ignacio Tinoco, and Carlos Bustamante. Reversible Unfolding of Single RNA Molecules by Mechanical Force. Science, 292(5517):733-737, April 2001.

[24] E. L. Florin, V. T. Moy, and H. E. Gaub. Adhesion forces between individual ligandreceptor pairs. Science, 264(5157):415-417, April 1994.

[25] R. Merkel, P. Nassoy, A. Leung, K. Ritchie, and E. Evans. Energy landscapes of receptor-ligand bonds explored with dynamic force spectroscopy. Nature, 397(6714):50-53, January 1999.

[26] Justin L. MacCallum, W. F. Drew Bennett, and D. Peter Tieleman. Distribution of Amino Acids in a Lipid Bilayer from Computer Simulations. Biophys J, 94(9):33933404, May 2008.

[27] Taras V. Pogorelov, Josh V. Vermaas, Mark J. Arcario, and Emad Tajkhorshid. Partitioning of Amino Acids into a Model Membrane: Capturing the Interface. The Journal of Physical Chemistry B, 118(6):1481-1492, February 2014. 
[28] Bryan T. Marshall, Mian Long, James W. Piper, Tadayuki Yago, Rodger P. McEver, and Cheng Zhu. Direct observation of catch bonds involving cell-adhesion molecules. Nature, 423(6936):190-193, May 2003.

[29] Evan Evans, Andrew Leung, Volkmar Heinrich, and Cheng Zhu. Mechanical switching and coupling between two dissociation pathways in a P-selectin adhesion bond. PNAS, 101(31):11281-11286, August 2004.

[30] Yuriy V. Pereverzev, Oleg V. Prezhdo, Manu Forero, Evgeni V. Sokurenko, and Wendy E. Thomas. The Two-Pathway Model for the Catch-Slip Transition in Biological Adhesion. Biophysical Journal, 89(3):1446-1454, September 2005.

[31] H. Risken. The Fokker-Planck Equation: Methods of Solution and Applications. Springer-Verlag Telos, 3rd edition, 1996.

[32] H. A. Kramers. Brownian motion in a field of force and the diffusion model of chemical reactions. Physica, 7(4):284-304, April 1940.

[33] Peter Hänggi, Peter Talkner, and Michal Borkovec. Reaction-rate theory: Fifty years after Kramers. Rev. Mod. Phys., 62(2):251, April 1990.

[34] D. B. Oliver and J. Beckwith. Identification of a new gene (seca) and gene product involved in the secretion of envelope proteins in escherichia coli. J Bacteriol, 150(2):686-91, 1982.

[35] Arnold J.M. Driessen and Nico Nouwen. Protein Translocation Across the Bacterial Cytoplasmic Membrane. Annual Review of Biochemistry, 77(1):643-667, June 2008. 
[36] Bahar T. Findik, Virginia F. Smith, and Linda L. Randall. Penetration into membrane of amino-terminal region of SecA when associated with SecYEG in active complexes: Penetration of SecA N Terminus into a Membrane Bilayer. Protein Science, 27(3):681-691, March 2018.

[37] W. Humphrey, A. Dalke, and K. Schulten. VMD: Visual molecular dynamics. J Mol Graph, 14(1):33-38, 27-28, February 1996.

[38] William L. Jorgensen, Jayaraman Chandrasekhar, Jeffry D. Madura, Roger W. Impey, and Michael L. Klein. Comparison of simple potential functions for simulating liquid water. The Journal of Chemical Physics, 79(2):926-935, July 1983.

[39] Jumin Lee, Xi Cheng, Jason M. Swails, Min Sun Yeom, Peter K. Eastman, Justin A. Lemkul, Shuai Wei, Joshua Buckner, Jong Cheol Jeong, Yifei Qi, Sunhwan Jo, Vijay S. Pande, David A. Case, Charles L. Brooks, Alexander D. MacKerell, Jeffery B. Klauda, and Wonpil Im. CHARMM-GUI Input Generator for NAMD, GROMACS, AMBER, OpenMM, and CHARMM/OpenMM Simulations Using the CHARMM36 Additive Force Field. Journal of Chemical Theory and Computation, 12(1):405-413, January 2016.

[40] Emilia L. Wu, Xi Cheng, Sunhwan Jo, Huan Rui, Kevin C. Song, Eder M. DávilaContreras, Yifei Qi, Jumin Lee, Viviana Monje-Galvan, Richard M. Venable, Jeffery B. Klauda, and Wonpil Im. CHARMM-GUI Membrane Builder toward realistic biological membrane simulations. Journal of Computational Chemistry, 35(27):1997-2004, October 2014.

[41] James C. Phillips, Rosemary Braun, Wei Wang, James Gumbart, Emad Tajkhorshid, Elizabeth Villa, Christophe Chipot, Robert D. Skeel, Laxmikant Kalé, and Klaus 
Schulten. Scalable molecular dynamics with NAMD. Journal of Computational Chemistry, 26(16):1781-1802, December 2005.

[42] Bernard R. Brooks, Robert E. Bruccoleri, Barry D. Olafson, David J. States, S. Swaminathan, and Martin Karplus. CHARMM: A program for macromolecular energy, minimization, and dynamics calculations. Journal of Computational Chemistry, 4(2):187-217, 1983.

[43] B. R. Brooks, C. L. Brooks, A. D. Mackerell, L. Nilsson, R. J. Petrella, B. Roux, Y. Won, G. Archontis, C. Bartels, S. Boresch, A. Caflisch, L. Caves, Q. Cui, A. R. Dinner, M. Feig, S. Fischer, J. Gao, M. Hodoscek, W. Im, K. Kuczera, T. Lazaridis, J. Ma, V. Ovchinnikov, E. Paci, R. W. Pastor, C. B. Post, J. Z. Pu, M. Schaefer, B. Tidor, R. M. Venable, H. L. Woodcock, X. Wu, W. Yang, D. M. York, and M. Karplus. CHARMM: The biomolecular simulation program. Journal of Computational Chemistry, 30(10):1545-1614, July 2009.

[44] Jeffery B. Klauda, Richard M. Venable, J. Alfredo Freites, Joseph W. O'Connor, Douglas J. Tobias, Carlos Mondragon-Ramirez, Igor Vorobyov, Alexander D. MacKerell, and Richard W. Pastor. Update of the CHARMM All-Atom Additive Force Field for Lipids: Validation on Six Lipid Types. The Journal of Physical Chemistry B, 114(23):7830-7843, June 2010.

[45] M. Tuckerman, B. J. Berne, and G. J. Martyna. Reversible multiple time scale molecular dynamics. The Journal of Chemical Physics, 97(3):1990-2001, August 1992.

[46] Qun Ma, Jesús A. Izaguirre, and Robert D. Skeel. Verlet-I/R-RESPA/Impulse is Limited by Nonlinear Instabilities. SIAM Journal on Scientific Computing, 24(6):1951-1973, January 2003. 
[47] Tom Darden, Darrin York, and Lee Pedersen. Particle mesh Ewald: An $N \cdot \log ($ $N$ ) method for Ewald sums in large systems. The Journal of Chemical Physics, 98(12):10089-10092, June 1993.

[48] H. J. C. Berendsen, J. P. M. Postma, W. F. van Gunsteren, A. DiNola, and J. R. Haak. Molecular dynamics with coupling to an external bath. The Journal of Chemical Physics, 81(8):3684-3690, October 1984.

[49] Scott E. Feller, Yuhong Zhang, Richard W. Pastor, and Bernard R. Brooks. Constant pressure molecular dynamics simulation: The Langevin piston method. The Journal of Chemical Physics, 103(11):4613-4621, September 1995.

[50] Naveen Michaud-Agrawal, Elizabeth J. Denning, Thomas B. Woolf, and Oliver Beckstein. MDAnalysis: A toolkit for the analysis of molecular dynamics simulations. Journal of Computational Chemistry, 32(10):2319-2327, July 2011.

[51] Richard J. Gowers, Max Linke, Jonathan Barnoud, Tyler JE Reddy, Manuel N. Melo, Sean L. Seyler, David L. Dotson, Jan Domanski, Sébastien Buchoux, and Ian M. Kenney. MDAnalysis: A Python package for the rapid analysis of molecular dynamics simulations. In Proceedings of the 15th Python in Science Conference, pages 98-105, 2016.

[52] Robert T. McGibbon, Kyle A. Beauchamp, Matthew P. Harrigan, Christoph Klein, Jason M. Swails, Carlos X. Hernández, Christian R. Schwantes, Lee-Ping Wang, Thomas J. Lane, and Vijay S. Pande. MDTraj: A Modern Open Library for the Analysis of Molecular Dynamics Trajectories. Biophysical Journal, 109(8):15281532, October 2015. 
[53] Wolfgang Kabsch and Christian Sander. Dictionary of protein secondary structure: Pattern recognition of hydrogen-bonded and geometrical features. Biopolymers, 22(12):2577-2637, December 1983.

[54] B. W. Bauer, T. Shemesh, Y. Chen, and T. A. Rapoport. A "push and slide" mechanism allows sequence-insensitive translocation of secretory proteins by the SecA ATPase. Cell, 157(6):1416-29, 2014.

[55] Hsuan-Liang Liu and Chia-Ming Hsu. The Effects of Various Alcohols on the Stability of Melittin: A Molecular Dynamics Study. Journal of the Chinese Chemical Society, 50(6):1235-1240, December 2003.

[56] Danilo Roccatano, Marco Fioroni, Martin Zacharias, and Giorgio Colombo. Effect of hexafluoroisopropanol alcohol on the structure of melittin: A molecular dynamics simulation study. Protein Science, 14(10):2582-2589, October 2005.

[57] T. V. Naumenkova, O. V. Levtsova, I. N. Nikolaev, and K. V. Shaitan. Comparative molecular dynamics study of the structural properties of melittin in water and trifluoroethanol/water. Biophysics, 55(1):24-28, February 2010.

[58] Alice Glättli, Indira Chandrasekhar, and Wilfred F. van. Gunsteren. A molecular dynamics study of the bee venom melittin in aqueous solution, in methanol, and inserted in a phospholipid bilayer. European Biophysics Journal, 35(3):255-267, February 2006.

[59] Sheeba J. Irudayam and Max L. Berkowitz. Influence of the arrangement and secondary structure of melittin peptides on the formation and stability of toroidal 
pores. Biochimica et Biophysica Acta (BBA) - Biomembranes, 1808(9):2258-2266, September 2011.

[60] T. C. Terwilliger and D. Eisenberg. The structure of melittin. I. Structure determination and partial refinement. J. Biol. Chem., 257(11):6010-6015, June 1982.

[61] Kalina Hristova, Christopher E. Dempsey, and Stephen H. White. Structure, Location, and Lipid Perturbations of Melittin at the Membrane Interface. Biophysical Journal, 80(2):801-811, February 2001.

[62] Stephen H White and William C Wimley. Hydrophobic interactions of peptides with membrane interfaces. Biochimica et Biophysica Acta (BBA) - Reviews on Biomembranes, 1376(3):339-352, November 1998.

[63] Jack Gauldie, Jennifer M. Hanson, Franklin D. Rumjanek, Rudolf A. Shipolini, and Charles A. Vernon. The Peptide Components of Bee Venom. European Journal of Biochemistry, 61(2):369-376, 1976.

[64] Horst Vogel. Incorporation of Melittin into phosphatidylcholine bilayers: Study of binding and conformational changes. FEBS Letters, 134(1):37-42, November 1981.

[65] Ellen Kuchinka and Joachim Seelig. Interaction of melittin with phosphatidylcholine membranes. Binding isotherm and lipid head-group conformation. Biochemistry, 28(10):4216-4221, May 1989.

[66] Delin Sun, Jan Forsman, and Clifford E. Woodward. Multistep Molecular Dynamics Simulations Identify the Highly Cooperative Activity of Melittin in Recognizing and Stabilizing Membrane Pores. Langmuir, 31(34):9388-9401, September 2015. 
[67] Sheeba J. Irudayam, Tobias Pobandt, and Max L. Berkowitz. Free Energy Barrier for Melittin Reorientation from a Membrane-Bound State to a Transmembrane State. The Journal of Physical Chemistry B, 117(43):13457-13463, October 2013.

[68] Gabriela Klocek, Therese Schulthess, Yechiel Shai, and Joachim Seelig. Thermodynamics of Melittin Binding to Lipid Bilayers. Aggregation and Pore Formation ${ }^{\dagger}$. Biochemistry, 48(12):2586-2596, March 2009.

[69] Geert van den Bogaart, Jeanette Velásquez Guzmán, Jacek T. Mika, and Bert Poolman. On the Mechanism of Pore Formation by Melittin. Journal of Biological Chemistry, 283(49):33854-33857, December 2008.

[70] Huey W. Huang. Molecular mechanism of antimicrobial peptides: The origin of cooperativity. Biochimica et Biophysica Acta (BBA) - Biomembranes, 1758(9):12921302, September 2006.

[71] Yuan Lyu, Xiao Zhu, Ning Xiang, and Ganesan Narsimhan. Molecular Dynamics Study of Pore Formation by Melittin in a 1,2-Dioleoyl- sn -glycero-3phosphocholine and 1,2-Di(9 Z -octadecenoyl)- $s n$-glycero-3-phospho-(1'- rac glycerol) Mixed Lipid Bilayer. Industrial $\mathcal{E}$ Engineering Chemistry Research, 54(42):10275-10283, October 2015.

[72] Yi Wang, Diana E. Schlamadinger, Judy E. Kim, and J. Andrew McCammon. Comparative molecular dynamics simulations of the antimicrobial peptide CM15 in model lipid bilayers. Biochimica et Biophysica Acta (BBA) - Biomembranes, 1818(5):1402-1409, May 2012. 
[73] Horst Vogel and Fritz Jähnig. Models for the structure of outer-membrane proteins of Escherichia coli derived from raman spectroscopy and prediction methods. Journal of Molecular Biology, 190(2):191-199, July 1986.

[74] David W. Hoskin and Ayyalusamy Ramamoorthy. Studies on anticancer activities of antimicrobial peptides. Biochimica et Biophysica Acta (BBA) - Biomembranes, 1778(2):357-375, February 2008.

[75] H. Raghuraman and Amitabha Chattopadhyay. Melittin: A Membrane-active Peptide with Diverse Functions. Bioscience Reports, 27(4-5):189-223, August 2007.

[76] S. V. Sharma. Melittin resistance: A counterselection for ras transformation. Oncogene, 7(2):193-201, February 1992.

[77] Shamsher S. Saini, Ashok K. Chopra, and Johnny W. Peterson. Melittin activates endogenous phospholipase D during cytolysis of human monocytic leukemia cells. Toxicon, 37(11):1605-1619, November 1999.

[78] S. V. Sharma. Melittin-induced hyperactivation of phospholipase A2 activity and calcium influx in ras-transformed cells. Oncogene, 8(4):939-947, April 1993.

[79] Islam Rady, Imtiaz A. Siddiqui, Mohamad Rady, and Hasan Mukhtar. Melittin, a major peptide component of bee venom, and its conjugates in cancer therapy. Cancer Letters, 402:16-31, August 2017.

[80] Fang-Yu Chen, Ming-Tao Lee, and Huey W. Huang. Evidence for Membrane Thinning Effect as the Mechanism for Peptide-Induced Pore Formation. Biophysical Journal, 84(6):3751-3758, June 2003. 
[81] Yuan Lyu, Ning Xiang, Xiao Zhu, and Ganesan Narsimhan. Potential of mean force for insertion of antimicrobial peptide melittin into a pore in mixed DOPC/DOPG lipid bilayer by molecular dynamics simulation. The Journal of Chemical Physics, 146(15):155101, April 2017.

[82] Jiaojiao Liu, Shufeng Xiao, Jingliang Li, Bing Yuan, Kai Yang, and Yuqiang Ma. Molecular details on the intermediate states of melittin action on a cell membrane. Biochimica et Biophysica Acta (BBA) - Biomembranes, 1860(11):2234-2241, November 2018.

[83] Yusuke Miyazaki, Susumu Okazaki, and Wataru Shinoda. Free energy analysis of membrane pore formation process in the presence of multiple melittin peptides. Biochimica et Biophysica Acta (BBA) - Biomembranes, page S0005273619300525, March 2019.

[84] Magnus Andersson, Jakob P. Ulmschneider, Martin B. Ulmschneider, and Stephen H. White. Conformational States of Melittin at a Bilayer Interface. Biophysical Journal, 104(6):L12-L14, March 2013.

[85] Charles H. Chen, Gregory Wiedman, Ayesha Khan, and Martin B. Ulmschneider. Absorption and folding of melittin onto lipid bilayer membranes via unbiased atomic detail microsecond molecular dynamics simulation. Biochimica et Biophysica Acta (BBA) - Biomembranes, 1838(9):2243-2249, September 2014.

[86] K. Matsuzaki, S. Yoneyama, and K. Miyajima. Pore formation and translocation of melittin. Biophysical Journal, 73(2):831-838, August 1997. 
[87] Maja Mihajlovic and Themis Lazaridis. Antimicrobial peptides in toroidal and cylindrical pores. Biochimica et Biophysica Acta (BBA) - Biomembranes, 1798(8):14851493, August 2010.

[88] Jung-Hsin Lin and A. Baumgaertner. Stability of a Melittin Pore in a Lipid Bilayer: A Molecular Dynamics Study. Biophysical Journal, 78(4):1714-1724, April 2000.

[89] Timothy C. Anglin, Krystal L. Brown, and John C. Conboy. Phospholipid flip-flop modulated by transmembrane peptides WALP and melittin. Journal of Structural Biology, 168(1):37-52, October 2009.

[90] Gregoria Illya and Markus Deserno. Coarse-Grained Simulation Studies of PeptideInduced Pore Formation. Biophysical Journal, 95(9):4163-4173, November 2008.

[91] Sheeba J. Irudayam and Max L. Berkowitz. Binding and reorientation of melittin in a POPC bilayer: Computer simulations. Biochimica et Biophysica Acta (BBA) Biomembranes, 1818(12):2975-2981, December 2012.

[92] Ziv Oren and Yechiel Shai. Selective Lysis of Bacteria but Not Mammalian Cells by Diastereomers of Melittin: Structure-Function Study ${ }^{\dagger}$. Biochemistry, 36(7):18261835, February 1997.

[93] Michal Sharon, Ziv Oren, Yechiel Shai, and Jacob Anglister. 2D-NMR and ATRFTIR Study of the Structure of a Cell-Selective Diastereomer of Melittin and Its Orientation in Phospholipids ${ }^{\dagger}$. Biochemistry, 38(46):15305-15316, November 1999.

[94] David Gfeller, Olivier Michielin, and Vincent Zoete. SwissSidechain: A molecular and structural database of non-natural sidechains. Nucleic Acids Research, 41(D1):D327-D332, October 2012. 
[95] David Gfeller, Olivier Michielin, and Vincent Zoete. Expanding molecular modeling and design tools to non-natural sidechains. J Comput Chem, 33(18):1525-1535, July 2012.

[96] Eric F. Pettersen, Thomas D. Goddard, Conrad C. Huang, Gregory S. Couch, Daniel M. Greenblatt, Elaine C. Meng, and Thomas E. Ferrin. UCSF Chimera?A visualization system for exploratory research and analysis. Journal of Computational Chemistry, 25(13):1605-1612, October 2004.

[97] Sunhwan Jo, Taehoon Kim, Vidyashankara G. Iyer, and Wonpil Im. CHARMMGUI: A web-based graphical user interface for CHARMM. Journal of Computational Chemistry, 29(11):1859-1865, August 2008.

[98] Emilia L. Wu, Xi Cheng, Sunhwan Jo, Huan Rui, Kevin C. Song, Eder M. DávilaContreras, Yifei Qi, Jumin Lee, Viviana Monje-Galvan, Richard M. Venable, Jeffery B. Klauda, and Wonpil Im. CHARMM-GUI Membrane Builder toward realistic biological membrane simulations. Journal of Computational Chemistry, 35(27):1997-2004, October 2014.

[99] Shuichi Nosé. A unified formulation of the constant temperature molecular dynamics methods. The Journal of Chemical Physics, 81(1):511, 1984.

[100] William G. Hoover. Canonical dynamics: Equilibrium phase-space distributions. Physical Review A, 31(3):1695-1697, March 1985.

[101] G.M. Torrie and J.P. Valleau. Nonphysical sampling distributions in Monte Carlo free-energy estimation: Umbrella sampling. Journal of Computational Physics, 23(2):187-199, February 1977. 
[102] Giacomo Fiorin, Michael L. Klein, and Jérôme Hénin. Using collective variables to drive molecular dynamics simulations. Molecular Physics, 111(22-23):3345-3362, December 2013.

[103] Shankar Kumar, John M. Rosenberg, Djamal Bouzida, Robert H. Swendsen, and Peter A. Kollman. THE weighted histogram analysis method for free-energy calculations on biomolecules. I. The method. Journal of Computational Chemistry, 13(8):1011-1021, October 1992.

[104] Dmitrij Frishman and Patrick Argos. Knowledge-based protein secondary structure assignment. Proteins: Structure, Function, and Genetics, 23(4):566-579, December 1995.

[105] Jeremy M. Berg, John L. Tymoczko, Lubert Stryer, Jeremy M. Berg, John L. Tymoczko, and Lubert Stryer. Biochemistry. W H Freeman, 5th edition, 2002.

[106] Glenn J. Martyna, Douglas J. Tobias, and Michael L. Klein. Constant pressure molecular dynamics algorithms. The Journal of Chemical Physics, 101(5):4177$4189,1994$.

[107] M. Connolly. Solvent-accessible surfaces of proteins and nucleic acids. Science, 221(4612):709-713, August 1983.

[108] Ana-Nicoleta Bondar and Stephen H. White. Hydrogen bond dynamics in membrane protein function. Biochimica et Biophysica Acta (BBA) - Biomembranes, 1818(4):942-950, April 2012.

[109] Edgar E. Kooijman, D. Peter Tieleman, Christa Testerink, Teun Munnik, Dirk T. S. Rijkers, Koert N. J. Burger, and Ben de Kruijff. An Electrostatic/Hydrogen Bond 
Switch as the Basis for the Specific Interaction of Phosphatidic Acid with Proteins. Journal of Biological Chemistry, 282(15):11356-11364, April 2007. 


\section{VITA}

Milica Utjesanovic was born in Sabac, Serbia in 1988. She graduated from University of Belgrade, in Belgrade, Serbia in 2012 with a Bachelor of Science degree in Physics. She graduated from from the University of Missouri in Columbia, MO in 2015 with a Master of Science degree in Physics. In 2015, she started her graduate research under the advisement of Dr. Ioan Kosztin at the University of Missouri and got a Ph.D. in Physics in 2019. During the time she was a Ph.D. candidate Milica welcomed her daughter, Nina. 